\title{
Categorical Liveness Checking by Corecursive Algebras
}

\author{
Natsuki Urabe* Masaki Hara \\ Dept. Computer Science, The University of Tokyo, Japan \\ \{urabenatsuki, qnighy\}@is.s.u-tokyo.ac.jp \\ *JSPS Research Fellow
}

\author{
Ichiro Hasuo \\ National Institute of Informatics, Japan \\ i.hasuo@acm.org
}

\begin{abstract}
Final coalgebras as "categorical greatest fixed points" play a central role in the theory of coalgebras. Somewhat analogously, most proof methods studied therein have focused on greatest fixed-point properties like safety and bisimilarity. Here we make a step towards categorical proof methods for least fixed-point properties over dynamical systems modeled as coalgebras. Concretely, we seek a categorical axiomatization of well-known proof methods for liveness, namely ranking functions (in nondeterministic settings) and ranking supermartingales (in probabilistic ones). We find an answer in a suitable combination of coalgebraic simulation (studied previously by the authors) and corecursive algebra as a classifier for (non-)well-foundedness.
\end{abstract}

\section{INTRODUCTION}

\section{A. Backgrounds}

Verification of liveness, much like that of safety, is a prototypical problem that underlines verification of more complex alternating fixed-point specifications. Liveness means that something "good" eventually occurs, while safety means that anything "bad" never occurs.

1) Ranking Functions: As an example, suppose that we are given a transition system as in the figure (1). Here $x_{1}$ is an accepting state that represents a good

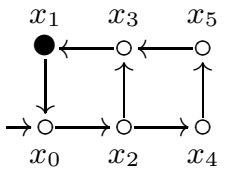
event. The reachability problem-a typical example of liveness checking problems - asks the following: "Does there exist a path from the initial state $x_{0}$ to $x_{1}$ ?" The answer is yes: $x_{1}$ is reachable by the path $\rightarrow x_{0} \rightarrow x_{2} \rightarrow x_{3} \rightarrow x_{1}$. Note that the path does not refer to the states $x_{4}$ and $x_{5}$.

In the example above, we assumed that the system is controlled in an angelic manner: we can choose the next state to eventually reach a good state.

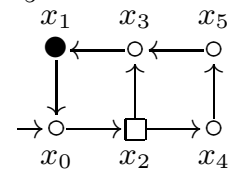

However, real-world systems often contain demonic branching, too, where the next state is chosen to avoid a good state. Such a system can be modeled as a two-player game played by angelic and demonic players. The figure (2) illustrates an example. At the state $x_{2}$ the next move is chosen by the demonic player. The answer to the reachability problem is again yes: no matter if $x_{3}$ or $x_{4}$ is chosen as the successor of $x_{2}$, the angelic player can force reaching $x_{1}$ (by $\rightarrow x_{3} \rightarrow x_{1}$ and $\rightarrow x_{4} \rightarrow x_{5} \rightarrow x_{3} \rightarrow x_{1}$ ).

Numerous methods are known for such liveness checking problems (e.g. [1], [2], [3]). A well-known method is the one using a ranking function [4]. For a two-player game, a ranking function is typically defined as a function $b: X \rightarrow \mathbb{N}_{\infty}$, from the state space $X$ to the set $\mathbb{N}_{\infty}=\mathbb{N} \cup\{\infty\}$, that satisfies the following conditions: (i) for each non-accepting state $x$ of the angelic player, there exists a successor state $x^{\prime}$ such that $b(x) \geq b\left(x^{\prime}\right)+1$; and (ii) for each non-accepting state $y$ of the demonic player, we have $b(y) \geq b\left(y^{\prime}\right)+1$ for each successor state $y^{\prime}$ of $y$. It is known that soundness holds: existence of a ranking function $b$ such that $b(x)<\infty$ implies that, regardless of the demonic player's choice, the angelic player can construct a path $x=x_{0} \rightarrow x_{1} \rightarrow \cdots$ that eventually reaches an accepting state. The well-foundedness of $\mathbb{N}$ is crucial here: we have $b\left(x_{i}\right)>b\left(x_{i+1}\right)$ for each $i$ before an accepting state is reached; and an infinite descending chain is impossible in $\mathbb{N}$. For example, in the two-player game in (2), the ranking function $b=\left[x_{0} \mapsto 5, x_{1} \mapsto 0, x_{2} \mapsto 4, x_{3} \mapsto 1, x_{4} \mapsto 3, x_{5} \mapsto 2\right]$ ensures that $x_{1}$ is reachable from $x_{0}$. Intuitively, the value $b(x)$ bounds the number of steps from $x$ to an accepting state.

2) Ranking Supermartingales: One can consider liveness checking problems also for probabilistic systems. A typical example is the almost-sure reachability problem: let us

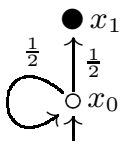
consider the probabilistic transition system (PTS) as in the figure (3). In the almost-sure reachability problem, we want to know if the accepting state $x_{1}$ is reached with probability 1. In the PTS in (3), the answer is yes, though there exists a path that does not visit any accepting state at all (namely $\rightarrow x_{0} \rightarrow x_{0} \rightarrow \cdots$, but this occurs with probability 0 ).

A notion analogous to that of ranking function is also known for probabilistic systems, namely ranking supermartingales [5], [6]. For a fixed positive real $\varepsilon>0$, an ( $\varepsilon$-additive) ranking supermartingale is a function $b^{\prime}: X \rightarrow[0, \infty]$, from the state space $X$ to the set $[0, \infty]$ of extended non-negative real numbers, that satisfies the following condition.

$\forall x \in X \backslash$ Acc. $b^{\prime}(x) \geq\left(\sum_{x^{\prime} \in X} \operatorname{Prob}\left(x \rightarrow x^{\prime}\right) \cdot b^{\prime}\left(x^{\prime}\right)\right)+\varepsilon$

Here $\operatorname{Prob}\left(x \rightarrow x^{\prime}\right)$ denotes the probability with which the system makes a transition from $x$ to $x^{\prime}$. This means that for each state $x \in X$, the expected value of $b^{\prime}$ decreases by at least $\varepsilon$ after a transition. The existence of a ranking supermartingale $b^{\prime}$ such that $b^{\prime}(x)<\infty$ implies that the expected value of the number of steps from $x$ to an accepting state is finite 


\begin{tabular}{c|c} 
functor $F$ & $c: X \rightarrow F X$ represents \\
\hline \hline$\left({ }^{2}\right)^{\Sigma} \times\{0,1\}$ & deterministic automaton \\
\hline $\mathcal{P}^{2}\left(\_\right) \times\{0,1\}$ & two-player game \\
\hline $\mathcal{D}\left(\_\right) \times\{0,1\}$ & probabilistic transition system (PTS)
\end{tabular}

Fig. 1. Coalgebraic representations of transition systems. Here $\mathcal{P}$ and $\mathcal{D}$ denote the powerset and the distribution functors respectively (Def. II.15.

(specifically it is no bigger than $b^{\prime}(x) / \varepsilon$ ). From this it easily follows that an accepting state is visited almost surely.

3) Coalgebras and Algebras: This paper aims to understand, in the categorical terms of (co)algebra, essences of liveness checking methods like ranking functions and ranking supermartingales. Coalgebras are commonly used for modeling state-based dynamics in the categorical language (see e.g. [7], [8]). Formally, for an endofunctor $F$ over a category $\mathbb{C}$, an $F$-coalgebra is an arrow $c$ of the type $c: X \rightarrow F X$. We can regard $X$ as a state space, $F$ as a specification of the branching type, and $c: X \rightarrow F X$ as a transition function. By changing the functor $F$ we can represent various kinds of transition types (see Fig. 1). It is also known that, using coalgebras, we can generalize various automata-theoretic notions and techniques (such as behavioral equivalence [9], bisimulation [10] and simulation [11]) to various systems (e.g. nondeterministic, probabilistic, and weighted ones).

A dual notion, i.e. an arrow of type $a: F X \rightarrow X$, is known as an $F$-algebra. In this paper, it is used to capture properties (or predicates) over a system represented as a coalgebra.

\section{B. Contributions}

We contribute a categorical axiomatization of "ranking functions" that is behind the well-known methods that we have sketched. It combines: corecursive algebras as value domains (that are, like $\mathbb{N}_{\infty}$, suited to detect well-foundedness) and lax homomorphisms (like in coalgebraic simulations [11], [12]). Based on the axiomatization we develop a general theory; our main result is soundness, i.e. that existence of a categorical ranking function indeed witnesses liveness (identified with a least fixed-point property). We also exploit our general theory and derive two new notions of "ranking functions" as instances. The two concrete definitions are new to the best of our knowledge.

We shall now briefly sketch our general theory, illustrating key notions and the backgrounds from which we derive them.

1) Corecursive Algebras for (Non-)Well-Foundedness: In the (conventional) definition of a ranking function $b: X \rightarrow$ $\mathbb{N}_{\infty}$, well-foundedness of $\mathbb{N}=\mathbb{N}_{\infty} \backslash\{\infty\}$ plays an important role as it ensures that no path can continue infinitely (without hitting an accepting state). Similarly, for a ranking supermartingale, it is crucial that $[0, \infty)=[0, \infty] \backslash\{\infty\}$ has no infinite sequence that decreases everywhere at least by $\varepsilon>0$. In unifying the two notions, we need to categorically capture well-foundedness.

Our answer comprises suitable use of core- $\quad F X \stackrel{F(|c|) r}{\rightarrow} F R$ cursive algebras [13]. An $F$-algebra $r: \uparrow_{c}=r \downarrow$ $F R \rightarrow R$ is said to be corecursive if from $\quad X \stackrel{(|c|)_{r}}{\rightarrow} R$ an arbitrary coalgebra $c: X \rightarrow F X$ there exists a unique coalgebra-algebra homomorphism $(c)_{r}$ (see the diagram). Corecursive algebras have been previously used to describe general structured corecursion [13] (see also Rem. II.17). Our use of them in this paper seems novel: $r$ being corecursive means that the function $\Phi_{c, r}: f \mapsto r \circ F f \circ c$ has a unique fixed point; in particular its least and greatest fixed points coincide 1 ; we find this feature of corecursive algebras suited for their use as categorical "classifiers" for (non-)well-foundedness.

2) Modalities and Least Fixed-Point Properties: Liveness properties such as reachability and termination are all instances of least fixed-point properties: once a proper modality $\nabla_{\sigma}$ is fixed, the property in question is described by a least fixedpoint formula $\mu u . \nabla_{\sigma} u$. The way we categorically formulate these constructs, as shown below, is nowadays standard (see e.g. [17], [18]). As the base category $\mathbb{C}$ we use Sets in this paper (although extensions e.g. to Meas would not be hard).

- We fix a domain $\Omega \in \mathbb{C}$ of truth values (e.g. $\Omega=\{0,1\}$ ), and a property over $X \in \mathbb{C}$ is an arrow $u: X \rightarrow \Omega$.

- A (state-based, dynamical) system is a coalgebra $c: X \rightarrow$ $F X$ for a suitable functor $F: \mathbb{C} \rightarrow \mathbb{C}$.

- A modality $\varnothing_{\sigma}$ is interpreted as an $F$-algebra $2 \sigma: F \Omega \rightarrow$ $\Omega$ over $\Omega$ (see Example III.4 and Prop. IV.2 for examples).

- Assuming some syntax is given, we should be able to derive the interpretation $\llbracket \nabla_{\sigma} \varphi \rrbracket_{c}$ of a modal formula from $\llbracket \varphi \rrbracket_{c}$. In the current (purely semantical) framework this goes as follows. Given a property $u: X \rightarrow \Omega$, we define the property $\Phi_{c, \sigma}(u): X \rightarrow \Omega$ by the composite

$$
\Phi_{c, \sigma}(u)=(X \stackrel{c}{\rightarrow} F X \stackrel{F u}{\rightarrow} F \Omega \stackrel{\sigma}{\rightarrow} \Omega) .
$$

- Assuming a suitable order structure $\sqsubseteq_{\Omega}$ on $\Omega$ and additional monotonicity requirements, the correspondence $\Phi_{c, \sigma}: \Omega^{X} \rightarrow \Omega^{X}$ has the least fixed point. It is denoted by $\llbracket \mu \sigma \rrbracket_{c}: X \rightarrow \Omega$; intuitively it is the interpretation $\llbracket \mu u . \varnothing_{\sigma} u \rrbracket_{c}$ of the formula $\mu u . \varnothing_{\sigma} u$ in the system $c$.

Concrete examples are in $₫$ III-B Another standard categorical modeling of a modality (see e.g. [19]) is by a predicate lifting, i.e. a natural transformation $\sigma_{X}: \Omega^{X} \Rightarrow \Omega^{F X}$. It corresponds to our modeling via the Yoneda lemma; see e.g. [17].

3) Ranking Functions, Categorically: Our modeling is summarized on the right: the liveness property $\llbracket \mu \sigma \rrbracket_{c}$ in question is the least arrow (with respect to the order on

$$
\begin{gathered}
F X \stackrel{F \llbracket \mu \sigma \rrbracket_{c}}{\longrightarrow} F \Omega \\
\uparrow c=\mu \\
X \underset{\llbracket \mu \sigma \rrbracket_{c}}{\longrightarrow} \Omega
\end{gathered}
$$

$\Omega$ ) that makes the square commute (note the subscript $={ }_{\mu}$ ).

The liveness checking problem is then formulated as follows: given an arrow $h: X \rightarrow \Omega$, we would like to decide if

\footnotetext{
${ }^{1}$ Examples abound in computer science-especially in domain theorywhere similar coincidences play important roles. They include: limit-colimit coincidence [14] and initial algebra-final coalgebra coincidence [15], [16].

2 We use the same functor $F$ for coalgebras (systems) and algebras (modalities). This characterization is used in [17], [18 and also found in many coalgebraic modal logic papers (e.g. [19]). However, for some examples, it comes more natural to use functors $F$ and $G$ together with a natural transformation $\alpha: F \Rightarrow G$, and to model a system and a modality as $c: X \rightarrow F X$ and $\sigma: G \Omega \rightarrow \Omega$ respectively. This modeling induces our current one as $\sigma$ and $\alpha$ together induce an $F$-algebra $F \Omega \stackrel{\alpha_{\Omega}}{\rightarrow} G \Omega \stackrel{\sigma}{\rightarrow} \Omega$ (cf. 乌ू).
} 
$h \sqsubseteq \Omega \llbracket \mu \sigma \rrbracket_{c}$ holds. Here $\sqsubseteq_{\Omega}$ denotes the pointwise extension of the order on $\Omega$. For example, let's say we want to check the assertion that a specific state $x_{0} \in X$ satisfies the liveness property $\llbracket \mu \sigma \rrbracket_{c}$. In this case we would define the above "assertion" $h: X \rightarrow \Omega$, where $\Omega=\{0 \sqsubseteq \Omega 1\}$, by: $h\left(x_{0}\right)=1$ and $h(x)=0$ for all $x \neq x_{0}$.

Our categorical framework of ranking function-based verification goes as follows.

- We fix a ranking domain - the value domain

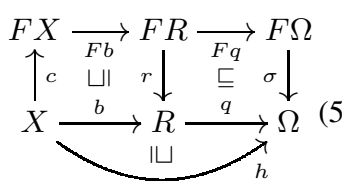

for ranking functions-to be an algebra $r: F R \rightarrow R$ together with a lax homomorphism $q: R \rightarrow \Omega$ (from $r$ to $\sigma$, in the right square in (5)). The latter $q$ identifies $r: F R \rightarrow R$ as a "refinement" of the modality $\sigma: F \Omega \rightarrow$ $\Omega$. A crucial requirement is that $r$ is corecursive, making it suited for detecting well-foundedness.

- A (categorical) ranking arrow for a coalgebra $c: X \rightarrow$ $F X$ is then defined to be a lax homomorphism $b: X \rightarrow$ $R$, in the sense shown in the left square in (5).

- Our soundness theorem says: given an assertion $h: X \rightarrow$ $\Omega$, in order to establish $h \sqsubseteq \llbracket \mu \sigma \rrbracket_{c}$, it suffices to find a ranking arrow $b: X \rightarrow R$ such that $h \sqsubseteq q \circ b$ (see (5)) .

This way the problem of verifying a least fixed-point property is reduced to finding a witness $b$. Note that the requirement on the ranking arrow $b$-namely $b \sqsubseteq r \circ F b \circ c$-is local (it only involves one-step transitions) and hence easy to check.

Our main technical contribution is a proof for soundness. There we argue in terms of inequalities between arrowsmuch like in [11], [12]-relying on fundamental ordertheoretic results on fixed points (Knaster-Tarski and CousotCousot, see $\llbracket$ II-D). Corecursiveness of $r$ is crucial there.

4) Concrete Examples: Ranking functions for two-player games $(₫-\mathrm{I})$ are easily seen to be an instance of our categorical notion, for suitable $F, \Omega$ and $R$.

Our second example in $₫$ I-A_-additive ranking supermartingales in the probabilistic setting-is itself not an example, however. Analyzing its reason we are led to a few variations of the definition, among which some seem new. We discuss these variations: their relationship, advantages and disadvantages.

\section{Organization of this Paper}

In $\oiiint$ we introduce preliminaries on: our running examples (two-player games and PTSs); liveness checking methods for them; (co)algebras; and least and greatest fixed points. Our main contribution is in $₫$ III where our categorical developments are accompanied (for illustration) by concrete examples from two-player games. The entailments of our general framework in the probabilistic setting are described in $₫[\mathrm{IV}$ Finally in $\$ \mathrm{~V}$ we conclude.

Some details and proofs are deferred to the appendices.

\section{PRELIMINARIES}

In this paper $[0, \infty)$ and $[0, \infty]$ denote the sets $\{a \in \mathbb{R}$ $a \geq 0\}$ and $\{a \in \mathbb{R} \mid a \geq 0\} \cup\{\infty\}$ respectively. We extend the ordinary order $\leq$ over $\mathbb{R}$ to $[0, \infty]$ by regarding $\infty$ as the greatest element. We write $\mathbb{N}_{\infty}$ for $\mathbb{N} \cup\{\infty\}$. For a function $\varphi: X \rightarrow[0,1]$, its support $\{x \in X \mid \varphi(x)>0\}$ is denoted by $\operatorname{supp}(\varphi)$.

\section{A. Two-player Games and Ranking Functions}

Our two-player games are played by an angelic player max and a demonic player min.

Definition II.1 (two-player game). A (two-player) game structure is a triple $G=\left(X_{\max }, X_{\min }, \tau\right)$ of a set $X_{\max }$ of states of the player max, a set $X_{\min }$ of states of the player min, and a transition relation $\tau \subseteq X_{\max } \times X_{\min } \cup X_{\min } \times X_{\max }$.

A strategy of the player $\max$ is a partial function $\alpha$ : $X_{\max } \times\left(X_{\min } \times X_{\max }\right)^{*} \rightarrow X_{\min }$ such that for each $x_{0} y_{1} x_{1} \ldots$ $y_{n} x_{n} \in X_{\max } \times\left(X_{\min } \times X_{\max }\right)^{*}$, if $\alpha\left(x_{0} y_{1} x_{1} \ldots y_{n} x_{n}\right)$ is defined then $\left(x_{n}, \alpha\left(x_{0} y_{1} x_{1} \ldots y_{n} x_{n}\right)\right) \in \tau$. A strategy of the player min is a partial function $\beta: X_{\max } \times\left(X_{\min } \times X_{\max }\right)^{*} \times$ $X_{\min } \rightarrow X_{\max }$ such that for each $x_{0} y_{1} x_{1} \ldots y_{n-1} x_{n-1} y_{n} \in$ $X_{\max } \times\left(X_{\min } \times X_{\max }\right)^{*} \times X_{\min }$, if $\beta\left(x_{0} y_{1} x_{1} \ldots y_{n-1} x_{n-1} y_{n}\right)$ is defined then $\left(y_{n}, \beta\left(x_{0} y_{1} x_{1} \ldots y_{n-1} x_{n-1} y_{n}\right)\right) \in \tau$.

For $x \in X_{\max }$ and a pair of strategies $\alpha$ and $\beta$ of the players $\max$ and min, respectively, the run induced by $\alpha$ and $\beta$ from $x$ is a possibly infinite sequence $\rho^{\alpha, \beta, x}=x_{0} y_{1} x_{1} y_{2} x_{2} \ldots$ that is an element of the set

$$
\begin{aligned}
& \left(X_{\max } \times\left(X_{\min } \times X_{\max }\right)^{*} \times\left\{\perp_{\max }\right\}\right) \\
& \cup\left(X_{\max } \times\left(X_{\min } \times X_{\max }\right)^{*} \times X_{\min } \times\left\{\perp_{\min }\right\}\right) \\
& \cup\left(X_{\max } \times X_{\min }\right)^{\omega}
\end{aligned}
$$

and is inductively defined as follows: for $n=0, x_{0}=x$; and for $n>0$,

$$
\begin{aligned}
& y_{n}= \begin{cases}\perp_{\max } \quad\left(\alpha\left(x_{0} y_{1} x_{1} \ldots y_{n-1} x_{n-1}\right) \text { is undefined }\right) \\
\alpha\left(x_{0} y_{1} x_{1} \ldots y_{n-1} x_{n-1}\right) \quad(\text { otherwise }), \text { and }\end{cases} \\
& x_{n}= \begin{cases}\perp_{\min } & \left(\beta\left(x_{0} y_{1} x_{1} \ldots x_{n-1} y_{n}\right) \text { is undefined }\right) \\
\beta\left(x_{0} y_{1} x_{1} \ldots x_{n-1} y_{n}\right) & (\text { otherwise })\end{cases}
\end{aligned}
$$

The symbol $\perp_{\max }$ (resp. $\perp_{\min }$ ) represents the end of the run at max's (resp. min's) turn: it means the player got stuck.

Once an initial state $x$ and strategies $\alpha$ and $\beta$ of the players $\max$ and min are fixed, a run $\rho^{\alpha, \beta, x}$ is determined. There are different ways to determine the "winner" of a run, including: $\max$ wins if min gets stuck; max wins if he does not get stuck; max wins if some specified states are visited infinitely many times (the Büchi condition), etc. In this paper where we focus on liveness, we choose the following (rather simple) winning condition: the player max wins if an accepting state is reached or the player min gets stuck. Studies of more complex conditions (like the Büchi condition) are left as future work.

Definition II.2 (reaching set). Let $G=\left(X_{\max }, X_{\min }, \tau\right)$ be a two-player game structure. We fix a set Acc $\subseteq X_{\max }$ of $a c$ cepting states. A run $\rho^{\alpha, \beta, x}=x_{0} y_{1} x_{1} \ldots$ on $\left(X_{\max }, X_{\min }, \tau\right)$ is winning with respect to Acc for the player max if

- $x_{n} \in$ Acc for some $n$; or

- $\rho^{\alpha, \beta, x}$ is a finite sequence whose last letter is $\perp_{\min }$. 
We define the reaching set $\operatorname{Reach}_{G, \text { Acc }} \subseteq X_{\max }$ by:

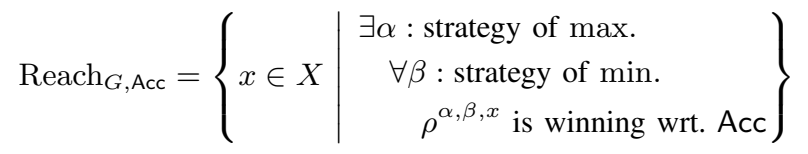

Example II.3. We define a game structure $G=\left(X_{\max }, X_{\min }, \tau\right)$ by $X_{\max }=$ $\left\{x_{0}, x_{1}, x_{2}, x_{3}, x_{4}\right\}, X_{\min }=\left\{y_{0}, y_{1}, y_{2}\right.$, $\left.y_{3}\right\}$ and $\tau=\left\{\left(x_{0}, y_{0}\right),\left(x_{0}, y_{1}\right),\left(x_{1}, y_{1}\right)\right.$ $\left(x_{1}, y_{2}\right),\left(x_{3}, y_{3}\right),\left(y_{0}, x_{2}\right),\left(y_{1}, x_{1}\right),\left(y_{1}, x_{2}\right)$,

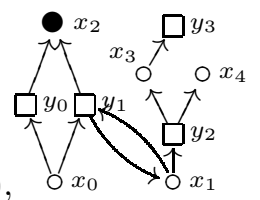
$\left.\left(y_{2}, x_{3}\right),\left(y_{2}, x_{4}\right)\right\}$. Let Acc $=\left\{x_{2}\right\}$. The situation is shown on the right. Then the reaching set is $\operatorname{Reach}_{G, \text { Acc }}=\left\{x_{0}, x_{2}, x_{3}\right\}$.

Ranking functions. Suppose that we are given a game structure $G=\left(X_{\max }, X_{\min }, \tau\right)$ and a set Acc $\subseteq X_{\max }$ of accepting states, and want to prove that a state $x$ is included in

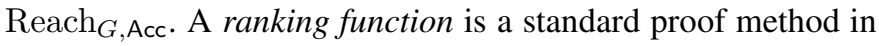
such a setting. There are variations in the definition of ranking function [4], [20], [21]: in this paper we use the following.

Definition II.4 (ranking function). Let $G=\left(X_{\max }, X_{\min }, \tau\right)$ be a game structure and Acc $\subseteq X_{\max }$. We fix an ordinal $\mathfrak{z}$ and let $\operatorname{Ord}_{\leq \mathfrak{z}}=\{\mathfrak{n} \mid \mathfrak{n} \leq \mathfrak{z}\}$ be the set of ordinals smaller than or equal to $\mathfrak{z}$. A function $b: X_{\max } \rightarrow \operatorname{Ord}_{\leq \mathfrak{z}}$ is called a ranking function (for $G$ and Acc) if it satisfies

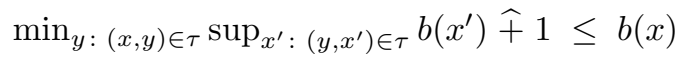

for each $x \in X_{\max } \backslash$ Acc, where $b\left(x^{\prime}\right) \widehat{+} 1=\min \left\{b\left(x^{\prime}\right)+1, \mathfrak{z}\right\}$ denotes addition truncated at $\mathfrak{z}$.

The following well-known theorem states soundness, i.e. that a ranking function witnesses reachability.

Theorem II.5 (soundness, see e.g. [4]). Let $\mathfrak{z}$ be an ordinal, and let $b: X \rightarrow \operatorname{Ord}_{\leq \mathfrak{z}}$ be a ranking function for $G$ and Acc. Then $b(x)<\mathfrak{z}$ (i.e. $b(x) \neq \mathfrak{z}$ ) implies $x \in \operatorname{Reach}_{G, \text { Acc. }}$

Example II.6. For the game in Example II.3. let $\mathfrak{z}=\omega$ and define a function $b: X \rightarrow \operatorname{Ord}_{\leq \mathfrak{z}}$ by $b\left(x_{0}\right)=1, b\left(x_{2}\right)=0$ and $b\left(x_{1}\right)=b\left(x_{3}\right)=b\left(x_{4}\right)=\omega$. Then $b$ is a ranking function. Hence by Thm. II.5, we have $x_{0} \in \operatorname{Reach}_{G, \text { Acc }}$.

Completeness (the converse of Thm. II.5) does not hold. A counterexample is given later in Example III.17.

Remark II.7. A strategy $\alpha: X_{\max } \times\left(X_{\min } \times X_{\max }\right)^{*} \rightarrow$ $X_{\min }$ is said to be positional if its outcome depends only on the last state of the input, i.e. $x_{n}=x_{n^{\prime}}^{\prime}$ implies $\alpha\left(x_{0} y_{1} \ldots y_{n} x_{n}\right)=\alpha\left(x_{0}^{\prime} y_{1}^{\prime} \ldots y_{n^{\prime}}^{\prime} x_{n^{\prime}}^{\prime}\right)$. It is known that a positional strategy suffices as long as we consider reaching sets, i.e. the set Reach $_{G, \text { Acc }}$ in Def. II.2 is unchanged if we replace " $\exists \alpha$ : strategy of max" in (6) with " $\exists \alpha$ : positional strategy of max" (see e.g. [22]).

A ranking function allows us to synthesize such a positional strategy. Let $x \in X_{\max }$ and $b: X_{\max } \rightarrow \operatorname{Ord}_{\leq_{\mathfrak{z}}}$ be a ranking function s.t. $b(x)<\infty$. We define a strategy $\alpha$ for max by

$$
\alpha\left(x_{0} y_{1} \ldots y_{n} x_{n}\right)=\arg \min _{y:\left(x_{n}, y\right) \in \tau} \sup _{x^{\prime}:\left(y, x^{\prime}\right) \in \tau} b\left(x^{\prime}\right) .
$$

Then it is a positional strategy such that for each strategy $\beta$ of min, the run $\rho^{\alpha, \beta, x}$ is winning wrt. Acc for max.

\section{B. Probabilistic Transition Systems and Ranking Supermartin- gales}

Definition II.8 (PTS). A probabilistic transition system (PTS) is a pair $M=(X, \tau)$ of a set $X$ and a transition function $\tau$ : $X \rightarrow \mathcal{D} X$. Here $\mathcal{D} X=\left\{d: X \rightarrow[0,1] \mid \sum_{x \in X} d(x)=1\right\}$ is the set of probability distributions over $X$.

Definition II.9 (reachability probability). Let $M=(X, \tau)$ be a PTS. We fix a set Acc $\subseteq X$ of accepting states. For each $x \in X$ and $n \in \mathbb{N}$, we define a value $f_{n}(x) \in[0,1]$ by:

$$
\begin{aligned}
& f_{n}(x)= \\
& \sum\left\{\begin{array}{l|l}
\prod_{i=0}^{k-1} \tau\left(x_{i}\right)\left(x_{i+1}\right) & \begin{array}{l}
k \leq n-1, x_{0}, \ldots, x_{k} \in X \\
x_{0}=x, x_{i} \notin \operatorname{Acc}(\forall i \in[0, k-1]), \\
\text { and } x_{k} \in \operatorname{Acc}
\end{array}
\end{array}\right\} .
\end{aligned}
$$

Note that if $x \in$ Acc then $f_{n}(x)=1$. As the sequence $\left(f_{n}(x)\right)_{n \in \mathbb{N}}$ is increasing for each $x \in X$, we can define a function $f: X \rightarrow[0,1]$ by:

$$
f(x)=\lim _{n \rightarrow \infty} f_{n}(x) .
$$

The function $f$ is called the reachability probability function with respect to $M$ and Acc, and is denoted by $\operatorname{Reach}_{M, \text { Acc }}$.

Here the value $f_{n}(x) \in[0,1]$ is the probability with which an accepting state is reached within $n$ steps from $x$.

Example II.10. We define a PTS $M=$ $(X, \tau)$ by $X=\left\{x_{0}, x_{1}, x_{2}, x_{3}\right\}$, and $\tau\left(x_{0}\right)=\left[x_{1} \mapsto \frac{1}{2}, x_{3} \mapsto \frac{1}{2}\right], \tau\left(x_{1}\right)=$ $\left[x_{1} \mapsto \frac{1}{2}, x_{2} \mapsto \frac{1}{2}\right], \tau\left(x_{2}\right)=\left[x_{2} \mapsto 1\right]$, and $\tau\left(x_{3}\right)=\left[x_{3} \mapsto 1\right]$. Let Acc $=$ $\left\{x_{2}\right\}$. The situation is as shown on the

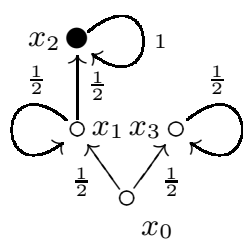
right. Then $\operatorname{Reach}_{M, \text { Acc }}: X \rightarrow[0,1]$ assigns $\frac{1}{2}$ to $x_{0}, 1$ to $x_{1}$ and $x_{2}$, and 0 to $x_{3}$.

Let us consider the almost-sure reachability problem for PTS. Given a PTS $M=(X, \tau)$, a set Acc $\subseteq X$ of accepting states and an (initial) state $x \in X$, we want to prove that $\operatorname{Reach}_{M, \operatorname{Acc}}(x)=1$. For this problem, a ranking function-like notion called ranking supermartingale [5] is known. There are several variations in its definition. We follow the definition in [6]; a variation can be found in [23].

Definition II.11 ( $\varepsilon$-additive ranking supermartingale). Let $M=(X, \tau)$ be a PTS and Acc $\subseteq X$ be the set of accepting states. Let $\varepsilon>0$ be a real number. A function $b^{\prime}: X \rightarrow[0, \infty]$ is an $\varepsilon$-additive ranking supermartingale (for $M$ and Acc) if

$$
\left(\sum_{x^{\prime} \in \operatorname{supp}(\tau(x))} \tau(x)\left(x^{\prime}\right) \cdot b^{\prime}\left(x^{\prime}\right)\right)+\varepsilon \leq b^{\prime}(x)
$$

holds for each $x \in X \backslash$ Acc.

Intuitively an $\varepsilon$-additive ranking supermartingale $b^{\prime}$ bounds the expected number of steps to accepting states: specifically it is no bigger than $b^{\prime}(x) / \varepsilon$. 
Theorem II.12 ([6]). Let $b^{\prime}: X \rightarrow[0, \infty]$ be an $\varepsilon$-additive ranking supermartingale for $M$ and Acc. Then $b^{\prime}(x)<\infty$ implies $\operatorname{Reach}_{M, \operatorname{Acc}}(x)=1$.

Example II.13. For the PTS in Example II.10, we define $b^{\prime}$ : $X \rightarrow[0, \infty]$ by $b^{\prime}\left(x_{0}\right)=\infty, b^{\prime}\left(x_{1}\right)=2, b^{\prime}\left(x_{2}\right)=0$ and $b^{\prime}\left(x_{3}\right)=\infty$. Then $b^{\prime}$ is a 1 -additive ranking supermartingale. Hence by Thm. II.12, we have $\operatorname{Reach}_{M, \operatorname{Acc}}\left(x_{1}\right)=1$.

\section{Categorical Preliminaries}

We assume that readers are familiar with basic categorical notions. For more details, see e.g. [24], [7].

Definition II.14 ((co)algebra). Let $F: \mathbb{C} \rightarrow \mathbb{C}$ be an endofunctor on a category $\mathbb{C}$. An $F$-coalgebra is a pair $(X, c)$ of an object $X$ in $\mathbb{C}$ and an arrow $c$ of the type $c: X \rightarrow F X$. An $F$-algebra is a pair $(A, a)$ of an object $A$ in $\mathbb{C}$ and an arrow $a$ of the type $a: F A \rightarrow A$.

In this paper we exclusively use the category Sets of sets and functions as the base category $\mathbb{C}$ (although extensions e.g. to Meas would not be hard). We would be interested in endofunctors composed by the following.

Definition II.15 $\left(\mathcal{P}, \mathcal{D}\right.$ and $\left.\left({ }_{-}\right) \times C\right)$. The powerset functor $\mathcal{P}:$ Sets $\rightarrow$ Sets is such that:

- for each $X \in$ Sets, $\mathcal{P} X=\{A \subseteq X\}$; and

- for each $f: X \rightarrow Y$ and $A \in \mathcal{P} X,(\mathcal{P} f)(A)=\{y \in Y \mid$ $\exists x \in A . f(x)=y\}$.

The (discrete) distribution functor $\mathcal{D}:$ Sets $\rightarrow$ Sets is:

- for each $X \in$ Sets, $\mathcal{D} X=\{d: X \rightarrow[0,1] \mid$ $\left.\sum_{x \in X} d(x)=1\right\} ;$ and

- for each $f: X \rightarrow Y, \delta \in \mathcal{D} X$ and $y \in Y,(\mathcal{D} f)(\delta)(y)=$ $\sum_{x \in f^{-1}(y)} \delta(x)$.

For $C \in$ Sets, the functor $\left({ }_{-}\right) \times C$ : Sets $\rightarrow$ Sets is:

- for $X \in$ Sets, $X \times C=\{(x, c) \mid x \in X, c \in C\}$; and

- for each $f: X \rightarrow Y, x \in X$ and $c \in C,(f \times C)(x, c)=$ $(f(x), c)$.

We combine these functors for modeling transition types of various kinds of systems (Fig. 11). For two-player games we use the functor $F_{\mathrm{g}}=\mathcal{P}^{2}\left(\_\right) \times\{0,1\}$. It works as follows.

- For each set $X, F_{\mathrm{g}} X=\{\Gamma \subseteq \mathcal{P} X\} \times\{0,1\}$.

- For each function $f: X \rightarrow Y, F_{\mathrm{g}} f: F_{\mathrm{g}} X \rightarrow F_{\mathrm{g}} Y$ is defined by $F_{\mathrm{g}} f(\Gamma, t)=(\{\{f(x) \mid x \in A\} \mid A \in \Gamma\}, t)$.

The correspondence between $F_{\mathrm{g}}$-coalgebras and two-player games will be spelled out in Def. III.1

The key idea in this paper is to use a corecursive algebra as a classifier for (non-)well-foundedness.

Definition II.16 (corecursive algebra, [13]). $\quad F X \stackrel{F f}{\rightarrow} F R$ An $F$-algebra $r: F R \rightarrow R$ is corecursive if $\quad \uparrow_{c}=r \downarrow$ given an arbitrary coalgebra $c: X \rightarrow F X, \quad X-\stackrel{f}{\rightarrow} \rightarrow R$ there exists a unique arrow $f: X \rightarrow R$ such that $f=r \circ F f \circ c$.

Remark II.17. The connection between corecursive algebras and (non-)well-foundedness has been hinted by some existing results. For example, for set functors preserving monos and inverse image diagrams, recursive coalgebras-the categorical dual of corecursive algebras used for general structured recursion in [25] - are known to coincide with well-founded coalgebras [26], where well-foundedness is categorically modeled in terms of "inductive components." For more general categories, it is known that if a functor preserves monos then well-foundedness implies recursiveness, but its converse does not necessarily hold [27]. The dual of this result, between corecursive and anti-founded algebras, is pursued in [13] but with limited success.

In [28] the notion of co-founded part of an algebra is introduced, with a main theorem that the co-founded part of an injectively structured corecursive algebra carries a final coalgebra. The result is used for characterizing a final coalgebra as that of suitable modal formulas. Despite its name, co-founded parts have little to do with our current view of corecursive algebras here as well-foundedness classifiers.

Discussions on other works on corecursive algebra are found in $9 \mathrm{~L}$ in the appendix.

\section{Verification of Least/Greatest Fixed-Point Properties}

The following results are fundamental in the studies of fixed-point specifications.

Theorem II.18. Let $L$ be a complete lattice, and $f: L \rightarrow L$ be a monotone function.

1) (Knaster-Tarski) The set of prefixed points (i.e. those $l \in L$ such that $f(l) \sqsubseteq l)$ forms a complete lattice. Moreover its least element is (not only a prefixed but) a fixed point, that is, the least fixed point $\mu f$.

2) (Cousot-Cousot [29]) Consider the (transfinite) sequence $\perp \sqsubseteq f(\perp) \sqsubseteq \cdots \sqsubseteq f^{\mathfrak{a}}(\perp) \sqsubseteq \cdots$ where, for a limit ordinal $\mathfrak{a}$, we define $f^{\mathfrak{a}}(\perp)=\bigsqcup_{\mathfrak{b}<\mathfrak{a}} f^{\mathfrak{b}}(\perp)$. The sequence eventually stabilizes and its limit is the least fixed point $\mu f$.

For the greatest fixed point $\nu f$ we have the dual results. From these four results-Knaster-Tarski and Cousot-Cousot, for $\mu$ and $\nu$-we derive the following four "proof principles."

Corollary II.19. Under the conditions of Thm. II.18.

$(\mathrm{KT} \mu) \quad f(l) \sqsubseteq l$ implies $\mu f \sqsubseteq l$.

$(\mathrm{KT} \nu) l \sqsubseteq f(l)$ implies $l \sqsubseteq \nu f$.

$(\mathrm{CC} \mu) f^{\mathfrak{a}}(\perp) \sqsubseteq \mu f$ for each ordinal $\mathfrak{a}$.

$(\mathrm{CC} \nu) \nu f \sqsubseteq f^{\mathfrak{a}}(\top)$ for each ordinal $\mathfrak{a}$.

Among these four, however, only two are applicable in verification: our goal is to show that an assertion $h$ is below a fixed point (see $[\mathrm{I-B} 3)$; the rules $(\mathrm{KT} \nu)$ and $(\mathrm{CC} \mu)$ are for under-approximation and thus serve our goal; but the other two are for over-approximating the fixed point in question.

It is these order-theoretic principles behind (namely CC and KT) that cause the difference between the proof methods for liveness (lfp's) and safety (gfp's). The role of ordinals $\mathfrak{a}$-equivalence classes of well-ordered sets-in $(\mathrm{CC} \mu)$ can be discerned in the definitions of ranking functions/supermartingales. These proof methods for liveness are 
in a sharp contrast with those for safety, in which finding an invariant (i.e. a post-fixed point $l$ in $(\mathrm{KT} \nu)$ ) suffices.

The basic idea behind the current contribution-liveness checking by combination of coalgebraic simulation and corecursive algebra - can be laid out as follows. For verification it is convenient if we can rely on certificates whose constraints are locally checkable. Their examples include invariants, various notions of (bi)simulation and a general notion of coalgebraic simulation; they are all postfixed points in a suitable sense. They should thus be able to witness only gfp's (not lfp's) in view of Cor. II.19. Here we leverage the lfpgfp coincidence in corecursive algebras to make coalgebraic simulations witness lfp's too. The lfp-gfp coincidence might seem a serious restriction but it is a common phenomenon in many "interesting" structures in computer science (as we discussed at the end of

\section{CATEGORICAL RANKING FUNCTIONS}

Here we present our general categorical framework for ranking function-based liveness checking.

\section{A. Running Example: Two-Player Games}

In this section, in order to provide abstract notions with intuitions, we use two-player games ( $₫$ II-A $)$ as a running example. We use the functor $F_{\mathrm{g}}=\mathcal{P}^{2}\left({ }_{-}\right) \times\{0,1\}:$ Sets $\rightarrow$ Sets to model them as coalgebras ( $₫$ II-C here g stands for "game").

Definition III.1. Given a $F_{\mathrm{g}}$-coalgebra $c: X \rightarrow F_{\mathrm{g}} X$, we define a game structure $G^{c}=\left(X_{\max }^{c}, X_{\min }^{c}, \tau^{c}\right)$ and a set $\mathrm{Acc}^{c} \subseteq X_{\max }$ of accepting states as follows: $X_{\max }^{c}=X$, $X_{\text {min }}^{c}=\mathcal{P} X, \tau^{c}=\left\{(x, A) \mid x \in X, A \in c_{1}(x)\right\} \cup\left\{\left(A, x^{\prime}\right) \mid\right.$ $\left.A \in \mathcal{P} X, x^{\prime} \in A\right\}$ and $\mathrm{Acc}^{c}=\left\{x \in X \mid c_{2}(x)=1\right\}$. Here we write $c(x)=\left(c_{1}(x), c_{2}(x)\right) \in \mathcal{P}^{2} X \times\{0,1\}$ for every $x$.

Conversely, given a game structure $G=\left(X_{\max }, X_{\min }, \tau\right)$ and a set Acc $\subseteq X_{\max }$, we define an $F_{\mathrm{g}}$-coalgebra $c^{G, \text { Acc }}$ : $X \rightarrow F_{\mathrm{g}} X$ as follows: $X=X_{\max }$ and $c^{G, \text { Acc }}(x)=\left(\left\{\left\{x^{\prime} \in\right.\right.\right.$ $\left.\left.\left.X_{\max } \mid\left(y, x^{\prime}\right) \in \tau\right\} \mid y \in X_{\min },(x, y) \in \tau\right\}, t\right)$ where $t$ is 1 if $x \in$ Acc and 0 otherwise.

The above two transformations constitute an embeddingprojection pair: games and $F_{\mathrm{g}}$-coalgebra are almost equivalent; the former have additional freedom (in the choice of the set $\left.X_{\min }\right)$ that is however inessential.

Throughout the rest of this section, each categorical notion is accompanied by a concrete example in terms of two-player games. For readability, the details of these examples (they are all straightforward) are deferred to $\mathrm{A}$ in the appendix. The other running example (PTSs) will be discussed later in

\section{B. Modalities and Least Fixed-Point Properties, Categorically}

Towards a categorical framework in which a soundness theorem is proved on the categorical level of abstraction, we need categorical modeling of modalities and least fixed-point properties. Our modeling here follows [17], [18]; it has been sketched in

The following function is heavily used in our developments.
Definition III.2 $\left(\Phi_{c, a}\right)$. Let $F$ : Sets $\rightarrow$ Sets, $c: X \rightarrow F X$ be a coalgebra and $a: F A \rightarrow A$ be an algebra. We define a function $\Phi_{c, a}: A^{X} \rightarrow A^{X}$ by $\Phi_{c, a}(f)=a \circ F f \circ c$, that is,

$$
(X \stackrel{f}{\longrightarrow} A) \stackrel{\Phi_{c, a}}{\longmapsto}\left(\begin{array}{cc}
F X \stackrel{F f}{\longrightarrow} F A \\
\uparrow c & a \downarrow \\
X & A
\end{array}\right) .
$$

Then corecursiveness (Def. II.16) is rephrased as follows: $r$ : $F R \rightarrow R$ is corecursive if and only if the function $\Phi_{c, r}$ has a unique fixed point for each $c: X \rightarrow F X$.

Our categorical modeling of modality is as follows.

Definition III.3 (a truth-value domain and an $F$-modality). A truth-value domain is a poset $\left(\Omega, \sqsubseteq_{\Omega}\right)$. If the order is clear from the context we simply write $\Omega$. For a functor $F$ : Sets $\rightarrow$ Sets, an $F$-modality over the truth-value domain $\Omega$ is an $F$ algebra $\sigma: F \Omega \rightarrow \Omega$.

Example III.4. For two-player games (i.e. $F_{\mathrm{g}}$-coalgebras) a natural truth-value domain is given by $(\{0,1\}, \leq)$ where 1 stands for "true." On top of this domain a natural $F_{\mathrm{g}}$-modality $\sigma_{\mathrm{g}}: F_{\mathrm{g}}\{0,1\} \rightarrow\{0,1\}$ is given as follows.

$$
\sigma_{\mathrm{g}}(\Gamma, t)= \begin{cases}1 & (t=1) \\ \max _{A \in \Gamma} \min _{a \in A} a & (\text { otherwise })\end{cases}
$$

Here, in $(\Gamma, t) \in F_{\mathrm{g}} X=\mathcal{P}^{2} X \times\{0,1\}, t \in\{0,1\}$ indicates if the current state is accepting or not ( $t=1$ if yes). The second case in the above definition of $\sigma_{\mathrm{g}}(\Gamma, t)$ reflects the intention that, in $\Gamma \in \mathcal{P}(\mathcal{P} X)$, the first $\mathcal{P}$ is for the angelic player max's choice while the second $\mathcal{P}$ is for the demonic min's.

Using an $F$-modality $\sigma$, liveness is categorically characterized as a least fixed-point property.

Definition III.5 $\left(\llbracket \mu \sigma \rrbracket_{c}\right)$. Let $\left(\Omega, \sqsubseteq_{\Omega}\right)$ be a truth-value domain and $\sigma: F \Omega \rightarrow \Omega$ be a modality. We say that $\sigma$ has least fixed points if for each $c: X \rightarrow F X$, the least fixed

$$
\begin{gathered}
F X \stackrel{F \llbracket \mu \sigma \rrbracket_{c}}{\longrightarrow} F \Omega \\
\uparrow c=\mu \\
X \underset{\llbracket \mu \sigma \rrbracket_{c}}{\longrightarrow} \Omega
\end{gathered}
$$
point of $\Phi_{c, \sigma}: \Omega^{X} \rightarrow \Omega^{X}$ (Def. III.2)-with respect to the pointwise extension of the order $\Xi_{\Omega}$ - exists. The least fixed point is called the (coalgebraic) least fixed-point property in $c$ specified by $\sigma$, and is denoted by $\llbracket \mu \sigma \rrbracket_{c}: X \rightarrow \Omega$.

Example III.6. The $F_{\mathrm{g}}$-modality $\sigma_{\mathrm{g}}: F_{\mathrm{g}}\{0,1\} \rightarrow\{0,1\}$ in Example III.4 has least fixed points (this follows from Prop. III.8 later). For each coalgebra $c: X \rightarrow F_{\mathrm{g}} X$, the least fixed-point property $\llbracket \mu \sigma_{\mathrm{g}} \rrbracket_{c}: X \rightarrow\{0,1\}$ is concretely described by:

$$
\llbracket \mu \sigma_{\mathrm{g}} \rrbracket_{c}(x)= \begin{cases}1 & \left(x \in \operatorname{Reach}_{\left.G^{c}, \operatorname{Acc}^{c}\right)}\right. \\ 0 & (\text { otherwise }) .\end{cases}
$$

Conversely, for each pair of a game structure $G$ and a set Acc, Reach ${ }_{G, \text { Acc }}$ is described by $\llbracket \mu \sigma_{\mathrm{g}} \rrbracket_{c}$,Acc. See Prop. A.2 for a proof. This way we characterize reachability in two-player games in categorical terms. 


\section{Ranking Domains and Ranking Arrows}

As we described in 4 -B33, we understand liveness checking as the task of determining if $h \sqsubseteq \llbracket \mu \sigma \rrbracket_{c}$, for a given assertion $h: X \rightarrow \Omega$. Here we introduce our categorical machinery for providing witnesses to such satisfaction of liveness.

For simplicity of arguments we assume the following.

Assumption III.7. Let $F:$ Sets $\rightarrow$ Sets. We assume that a truth-value domain $(\Omega, \sqsubseteq \Omega)$ and an $F$-modality $\sigma: F \Omega \rightarrow \Omega$ over $\Omega$ satisfy the following conditions.

1) The poset $(\Omega, \sqsubseteq \Omega)$ is a complete lattice.

2) For each $F$-coalgebra $c: X \rightarrow F X$, the function $\Phi_{c, \sigma}$ : $\Omega^{X} \rightarrow \Omega^{X}$ in Def. III.5 is monotone with respect to the pointwise extension of $\sqsubseteq_{\Omega}$.

These assumptions are mild. For example, Cond. 2 is satisfied if: $F \Omega$ has an order structure; $\sigma: F \Omega \rightarrow \Omega$ is monotone; and the action $F_{X, \Omega}: \Omega^{X} \rightarrow(F \Omega)^{F X}$ of $F$ on arrows is monotone, too. Cond. 1 in the above implies that $\Omega^{X}$ is a complete lattice. Thus we can construct a transfinite sequence $\perp_{\Omega} \sqsubseteq \Phi_{c, \sigma}\left(\perp_{\Omega}\right) \sqsubseteq \cdots \sqsubseteq \Phi_{c, \sigma}^{\mathfrak{a}}\left(\perp_{\Omega}\right) \sqsubseteq \cdots$ as in Thm. \.18, 2 , to obtain the least fixed point of $\Phi_{c, \sigma}$ as its limit.

Proposition III.8. Under the conditions in Asm. III.7 $\sigma$ has least fixed points (in the sense of Def. [II.5).

Example III.9. The data $F_{\mathrm{g}}, \sigma_{\mathrm{g}}$ for two-player games satisfy the assumptions: see Prop. A.3 (in the appendix) for a proof.

We are ready to introduce the key notions.

Definition III.10 (ranking domains). We assume Asm. III.7 Let $r: F R \rightarrow R$ be an $F$-algebra, $q: R \rightarrow \Omega$ be an arrow, and $\sqsubseteq_{R}$ be a partial order on $R$. Note that for each set $X$, the order $\sqsubseteq_{\Omega}$ (resp. $\sqsubseteq_{R}$ ) extends to the one between functions $X \rightarrow \Omega$ (resp. $X \rightarrow R$ ) in a pointwise manner.

A triple $\left(r, q, \sqsubseteq_{R}\right)$ is called a ranking domain for $F$ and $\sigma$ if the following conditions are

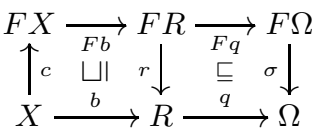
satisfied.

1) We have $q \circ r \sqsubseteq \Omega \sigma \circ F q$ between arrows $F R \rightarrow \Omega$ (the square on the right in (7)).

2) The same conditions as in Asm. $\amalg I .7$ hold for $r$, i.e.

a) the poset $\left(R, \sqsubseteq_{R}\right)$ is a complete lattice; and

b) for each $c: X \rightarrow F X$, the function $\Phi_{c, r}: R^{X} \rightarrow$ $R^{X}$ (Def. III.2) is monotone.

3) The function $q: R \rightarrow \Omega$ is monotone (i.e. $a \sqsubseteq_{R} b \Rightarrow$ $q(a) \sqsubseteq_{\Omega} q(b)$ ), strict (i.e. $q\left(\perp_{R}\right)=\perp_{\Omega}$ ) and continuous (i.e. for each subset $K \subseteq R$, we have $q\left(\bigsqcup_{a \in K} a\right)=$ $\bigsqcup_{a \in K} q(a)$ ).

4) The algebra $r: F R \rightarrow R$ is corecursive.

Cond. 2 in the definition ensures that the least fixed point of $\Phi_{c, r}$ arises from the approximation sequence in Thm. [I.18,2. Cond. 3 ensures that this least fixed point is preserved by $q$. In particular we insist on strictness-this is much like in domain theory [30]. The most significant in Def. III.10] is the corecursiveness of $r$ (Cond. 4): it makes $r$ a refinement of $\sigma$ that is suited for detecting well-foundedness.

Definition III.11 (ranking arrows). Let $\left(r, q, \sqsubseteq_{R}\right)$ be a ranking domain for $F$ and $\sigma$; and $c: X \rightarrow F X$ be a coalgebra. An arrow $b: X \rightarrow R$ is called a (coalgebraic) ranking arrow for $c$ with respect to $\left(r, q, \sqsubseteq_{R}\right)$ if it satisfies $b \sqsubseteq_{R} \Phi_{c, r}(b)=r \circ F b \circ c$ (the square on the left in (7)).

Now we give a soundness theorem for (categorical) ranking arrows. This is the main theorem of this paper; its proof demonstrates the role of the corecursiveness assumption.

Theorem III.12 (soundness). Let $\left(r, q, \sqsubseteq_{R}\right)$ be a ranking domain. Let $c: X \rightarrow F X$ be an $F$-coalgebra and $b: X \rightarrow R$ be a ranking arrow for $c$ (i.e. $b \sqsubseteq$ $r \circ F b \circ c)$. Then we have:

$$
q \circ b \sqsubseteq_{\Omega} \llbracket \mu \sigma \rrbracket_{c} .
$$

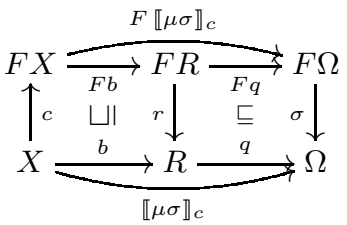

Thus for liveness checking (i.e. for proving $h \sqsubseteq \llbracket \mu \sigma \rrbracket_{c}$ ) it suffices to find a ranking arrow $b$ such that $h \sqsubseteq q \circ b$. In the proof of the theorem we use the following generalization of Thm. II.18 2. It starts from a post-fixed point $l$ (not from $\perp$ ).

Lemma III.13. Assume the conditions in Thm. II.18 and let $l$ be a post-fixed point of $f$, i.e. $l \sqsubseteq f(l)$. Then we can define a transfinite sequence $l \sqsubseteq f(l) \sqsubseteq \cdots \sqsubseteq f^{\mathfrak{a}}(l) \sqsubseteq \cdots$ in a similar manner to Thm. II.18 2. The sequence eventually stabilizes and its limit is a (not necessarily least) fixed point of $f$.

Proof (Thm. III.12). By Cond. 2a in Def. III.10, the poset $\left(R^{X}, \sqsubseteq_{R}\right)$ is a complete lattice. Moreover, by its definition, $b: X \rightarrow R$ is a post-fixed point of $\Phi_{c, r}$. Hence together with Cond. 2b we can construct a transfinite sequence $b \sqsubseteq_{R}$ $\Phi_{c, r}(b) \sqsubseteq_{R} \cdots \sqsubseteq_{R} \Phi_{c, r}^{\mathfrak{a}}(b) \sqsubseteq_{R} \cdots$ as in Lem. 【II.13 By Lem. III.13, there exists an ordinal $\mathfrak{m}$ such that $\Phi_{c, r}^{\mathfrak{m}}(b)$ is a fixed point of $\Phi_{c, r}$. By its definition, we have $b \sqsubseteq_{R} \Phi_{c, r}^{\mathfrak{m}}(b)$.

Note here that $r$ is assumed to be a corecursive algebra (Cond. 44 in Def. III.10). Hence $\Phi_{c, \sigma}$ has a unique fixed point; it is denoted by $(c)_{r}: X \rightarrow R$. Then we have:

$$
b \sqsubseteq_{R} \Phi_{c, r}^{\mathfrak{m}}(b)=(c)_{r} .
$$

By Cond.2a in Def. III.10, $R^{X}$ is a complete lattice. Hence we can also define $\Phi_{c, r}^{\mathfrak{a}}\left(\perp_{R}\right): X \rightarrow$ $R$ for each ordinal $\mathfrak{a}$ (here $\perp_{R}$ denotes the least element in $R^{X}$ ), and by Thm. II.18 2, there exists

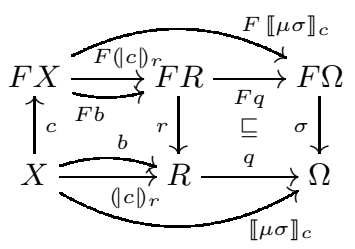
$\mathfrak{m}^{\prime}$ such that $\Phi_{c, r}^{\mathfrak{m}^{\prime}}\left(\perp_{R}\right)$ is also a fixed point of $\Phi_{c, r}$. Hence $\left.\Phi_{c, r}^{\mathfrak{m}^{\prime}}\left(\perp_{R}\right)=(c)\right)_{r}$.

We shall now prove that $q \circ \Phi_{c, r}^{\mathfrak{a}}\left(\perp_{R}\right) \sqsubseteq \llbracket \mu \sigma \rrbracket_{c}$ holds for each ordinal $\mathfrak{a}$. This is by transfinite induction on $\mathfrak{a}$.

For $\mathfrak{a}=0$, we have:

$$
\begin{array}{rlr}
q \circ \Phi_{c, r}^{\mathfrak{a}}\left(\perp_{R}\right) & =\quad q \circ \perp_{R} & \text { (by definition) } \\
& =\quad \perp_{\Omega} & \text { (by Cond. 3 } 3 \text { in Def. 【II.10) } \\
& \sqsubseteq_{\Omega} \llbracket \mu \sigma \rrbracket_{c} . &
\end{array}
$$


For a successor ordinal $\mathfrak{a}+1$, we have:

$$
\begin{aligned}
& q \circ \Phi_{c, r}^{\mathfrak{a}+1}\left(\perp_{R}\right) \\
& =\quad q \circ r \circ F\left(\Phi_{c, r}^{\mathfrak{a}}\left(\perp_{R}\right)\right) \circ c \\
& \sqsubseteq_{\Omega} \sigma \circ F\left(q \circ \Phi_{c, r}^{\mathfrak{a}}\left(\perp_{R}\right)\right) \circ c \\
& \sqsubseteq_{\Omega} \sigma \circ F\left(\llbracket \mu \sigma \rrbracket_{c}\right) \circ c \\
& =\quad \llbracket \mu \sigma \rrbracket_{c} .
\end{aligned}
$$

(by definition)

(by Cond. 1 in Def. III.10)

(by IH and Asm. III.7/2)

$\left(\llbracket \mu \sigma \rrbracket_{c}\right.$ is a fixed point $)$
For a limit ordinal $\mathfrak{l}$, we have:

$$
\begin{array}{lr}
q \circ \Phi_{c, r}^{\mathfrak{l}}\left(\perp_{R}\right) & \\
=q\left(\bigsqcup_{\mathfrak{a}<\mathfrak{l}} \Phi_{c, r}^{\mathfrak{a}}\left(\perp_{R}\right)\right) & \text { (by definition)) } \\
=\bigsqcup_{\mathfrak{a}<\mathfrak{l}}\left(q \circ \Phi_{c, r}^{\mathfrak{a}}\left(\perp_{R}\right)\right) & \text { (by Cond. 3 in Def. 【II.10) } \\
\sqsubseteq \Omega \llbracket \mu \sigma \rrbracket_{c} & \text { (by IH). }
\end{array}
$$

As $\Phi_{c, r}^{\mathfrak{m}^{\prime}}\left(\perp_{R}\right)=(|c|)_{r}$, the last fact yields $q \circ(\mid c)_{r} \sqsubseteq \llbracket \mu \sigma \rrbracket_{c}$. Combining with (8) and the monotonicity of $q$, we obtain $q \circ$ $b \sqsubseteq q \circ(\mid c)_{r} \sqsubseteq \llbracket \mu \sigma \rrbracket_{c}$. This concludes the proof.

Remark III.14. Note that the requirement on ranking arrows- $b \sqsubseteq_{R} r \circ F b \circ c$ (Def. III.11) — is a local one: it only involves one-step transitions by $c$ and hence is easy to check.

The condition asserts that $b$ is a suitable post-fixed point. In view of the order-theoretic foundations in $\oiiint$ II-D this might seem strange: we are using an invariant-like construct $b$ to witness a least fixed point, not a greatest. We are allowed to do so thanks to the corecursiveness of $r: F R \rightarrow R$ here the least and greatest fixed points for $\Phi_{c, r}$ coincide. It is also crucial that $q$ preserves least fixed points, being strict and continuous.

Example III.15. For two-player games as $F_{\mathrm{g}}$-coalgebras, we can define a ranking domain

$$
\left(r_{\mathrm{g}, \mathfrak{z}}: F_{\mathrm{g}} \operatorname{Ord}_{\leq \mathfrak{z}} \rightarrow \operatorname{Ord}_{\leq \mathfrak{z}}, q_{\mathrm{g}, \mathfrak{z}}: \operatorname{Ord}_{\leq \mathfrak{z}} \rightarrow\{0,1\}, \sqsubseteq \text { Ord }\right)
$$

as follows.

1) $\operatorname{Ord}_{\leq \mathfrak{z}}=\{\mathfrak{a} \mid \mathfrak{a}$ is an ordinal s.t. $\mathfrak{a} \leq \mathfrak{z}\}$, and

$$
r_{\mathrm{g}, \mathfrak{z}}(\Gamma, t)= \begin{cases}0 & (t=1) \\ \min _{A \in \Gamma} \sup _{\mathfrak{a} \in A}(\mathfrak{a} \widehat{+} 1) & (\text { otherwise }) .\end{cases}
$$

2) $q_{\mathrm{g}, \mathfrak{z}}(\mathfrak{z})=0$, and $q_{\mathfrak{g}, \mathfrak{z}}(\mathfrak{a})=1$ for any $\mathfrak{a}$ such that $\mathfrak{a}<\mathfrak{z}$;

3) $\mathfrak{a} \sqsubseteq$ Ord $\mathfrak{b} \stackrel{\text { def. }}{\Leftrightarrow} \mathfrak{a} \geq \mathfrak{b}$ (note the directions of inequalities).

Recall that $\mathfrak{a} \widehat{+} 1$ denotes $\min \{\mathfrak{a}+1, \mathfrak{z}\}$. The triple $\left(r_{\mathrm{g}, \mathfrak{z}}, q_{\mathrm{g}, \mathfrak{z}}\right.$, ○ord $)$ is indeed a ranking domain (see Prop. A.4 in the appendix). One can think of the above data as a classifier for (non-)well-foundedness: all the ordinals $\mathfrak{a}<\mathfrak{z}$ are for "well-founded" and the maximum ordinal $\mathfrak{z}$ is for "non-wellfounded." Observe that the map $q_{\mathrm{g}, \mathfrak{z}}$ acts accordingly.

We indeed have the following correspondences.

a) $b: X \rightarrow \operatorname{Ord}_{\leq \mathfrak{z}}$ is a (categorical) ranking arrow (Def. III.11) for an $F_{\mathrm{g}}$-coalgebra $c: X \rightarrow F_{\mathrm{g}} X$ wrt. $\left(r_{\mathrm{g}, \mathfrak{z}}, q_{\mathrm{g}, \mathfrak{z}}, \sqsubseteq\right.$ Ord $)$ iff $b$ is a ranking function for $G^{c}$ and $\mathrm{Acc}^{c}$ (in the conventional sense of Def. II.4). b) $b: X \rightarrow$ Ord $_{\leq \mathfrak{z}}$ is a ranking function for a game structure $G$ and a set Acc iff $b$ is a ranking arrow for $c^{G, \text { Acc }}$ wrt. $\left(r_{\mathrm{g}, \mathfrak{z}}, q_{\mathrm{g}, \mathfrak{z}}, \sqsubseteq\right.$ Ord $)$.

c) $b(x)<\mathfrak{z}$ iff $q_{\mathrm{g}, \mathfrak{z}} \circ b(x)=1$.

Here recall the correspondence in Def. III.1. A formal statement and its proof are found in Prop. A.7

Combined with the characterization in Example III.6, we conclude that the conventional soundness result (Thm. II.5) is an instance of our categorical soundness (Thm. III.12).

Remark III.16. Assume the conditions in Thm. III.12 As $r: F R \rightarrow R$ is a corecursive algebra, there exists a unique arrow $(|c|)_{r}: X \rightarrow R$ such that $(|c|)_{r}=r \circ F(|c|)_{r} \circ c$. Since $(|c|)_{r}$ is obviously the greatest fixed point of $\Phi_{c, \sigma}$, by the KnasterTarski theorem (cf. Thm. II.18), for each (categorical) ranking arrow $b: X \rightarrow R$ we have $b \sqsubseteq_{R}(c)_{r}$. This means that if $h \sqsubseteq \Omega q \circ b$ then $h \sqsubseteq \Omega q \circ(c)_{r}$. Therefore we can say that the unique arrow $(c)_{r}: X \rightarrow R$ is the "optimal" ranking arrow in the sense that if $(c)_{r}$ cannot prove liveness then no (categorical) ranking arrow can prove liveness using $R$ and $q$, either. For two-player games, the optimal ranking arrow is given by the function assigning a state $x$ the minimum number of steps from $x$ to Acc.

We note that the converse of Thm. III.12 (i.e. completeness) does not necessarily hold. In other words, it is possible that there exists no ranking arrow $b: X \rightarrow R$ such that $q \circ b=$ $\llbracket \mu \sigma \rrbracket_{c}$. Here is a counterexample.

Example III.17. We define an $F_{\mathrm{g}}$-coalgebra $c: X \rightarrow F_{\mathrm{g}} X$ by $X=\left\{x_{\mathfrak{a}} \mid \mathfrak{a} \leq \omega\right\}, c\left(x_{0}\right)=$ $(\emptyset, 1)$ and $c\left(x_{\mathfrak{a}}\right)=\left(\left\{\left\{x_{\mathfrak{b}} \mid \mathfrak{b}<\right.\right.\right.$

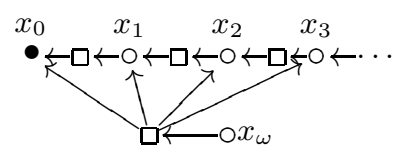
$\mathfrak{a}\}\}, 0)$ for each $\mathfrak{a}>0$. Note that in the corresponding game structure $G_{c}$, all the choices are made by the player min. Then we have $\llbracket \mu \sigma_{\mathrm{g}} \rrbracket_{c}\left(x_{\omega}\right)=1$ because of well-foundedness of $\omega$. However, the unique arrow $(c)_{r_{\mathrm{g}, \omega}}: X \rightarrow \mathbf{O r d}_{\leq \omega}$ such that $(c \mid)_{r_{\mathrm{g}, \omega}}=\Phi_{c, \sigma_{\mathrm{g}}}\left((|c|)_{r_{\mathrm{g}, \omega}}\right)$ assigns, to each state $x_{\mathfrak{a}}$, the ordinal $\mathfrak{a}$. This means that $q_{\mathrm{g}, \omega} \circ(|c|)_{r_{\mathrm{g}, \omega}}\left(x_{\omega}\right)=q_{\mathrm{g}, \omega}(\omega)=0$. Thus $q_{\mathrm{g}, \omega} \circ(|c|)_{r_{\mathrm{g}, \omega}}<\llbracket \mu \sigma_{\mathrm{g}} \rrbracket_{c}$.

Similarly, for every ordinal number $\mathfrak{z}$, we can construct an $F_{\mathrm{g}}$-coalgebra whose reachability cannot be proved by the ranking domain $r_{\mathrm{g}, \mathfrak{z}}: F_{\mathrm{g}} \mathbf{O r d}_{\leq \mathfrak{z}} \rightarrow \operatorname{Ord}_{\leq \mathfrak{z}}$.

By cardinality arguments we can show that sort of "completeness" holds in the example above, in the following sense: for every $F_{\mathrm{g}}$-coalgebra $c$ there exists an ordinal $\mathfrak{z}$ such that the reachability of $c$ is provable by the ranking domain $r_{\mathrm{g}, \mathfrak{z}}$. However, in this paper we use the term "completeness" in a different sense in which we fix the domain $R$ of ranking functions in advance.

Here is a categorical sufficient condition for completeness.

Proposition III.18 (a sufficient condition for completeness). Let $\left(r, q, \sqsubseteq_{R}\right)$ be a ranking domain, $c: X \rightarrow F X$ be an $F$-coalgebra, and $(\mid c)_{r}: X \rightarrow R$ be the unique arrow such 
that $(\mid c)_{r}=\left.r \circ F(c)\right|_{r} \circ c$. Assume that we have the equality

$$
q \circ r=\sigma \circ F q \text {, }
$$

instead of an inequality, in the

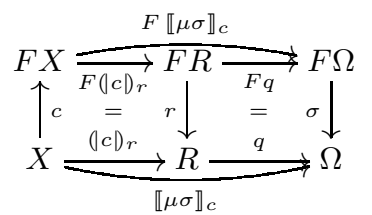
square on the right. Then we have $q \circ(\mid c)_{r}=\llbracket \mu \sigma \rrbracket_{c}$.

Intuitively, the equality (9) means that $r$ approximates the modality $\sigma$ in an adequate way. The result implies that, in case $h: X \rightarrow \Omega$ satisfies $h \sqsubseteq \llbracket \mu \sigma \rrbracket_{c}$, the latter inequality can always be witnessed by some ranking arrow (namely $\left.(c)_{r}\right)$. This is completeness of the proof method of categorical ranking arrows. An example of a complete ranking domain will be given in

\section{CATEgorical Ranking ARrows FOR PROBABILISTIC TRANSITION SYSTEMS}

We shall now investigate what our categorical framework in $\leftrightarrows$ III entails in the probabilistic setting of $\coprod$ II-B It turns out that the well-known definition of ranking supermartingale $(\varepsilon$ additive ones in Def. II.11) is not an instance. Here we study some variations of the definition of ranking supermartingale; two among them (distribution-valued and non-counting ones, that are new to our knowledge) exhibit the nice categorical properties in III We also discuss some relationships between those variations, showing that the soundness of $\varepsilon$-additive ranking supermartingales (Def. II.11) can nevertheless be proved via the categorical arguments in

\section{A. Probabilistic Transition Systems as Coalgebras}

To represent a PTS as a coalgebra, we use the functor $F_{\mathrm{p}}$ : Sets $\rightarrow$ Sets (p stands for "probability") defined as follows.

Definition IV.1 $\left(F_{\mathrm{p}}\right)$. We let $F_{\mathrm{p}}=\mathcal{D}\left({ }_{-}\right) \times\{0,1\}$, where $\mathcal{D}$ is the (discrete) distribution functor in Def. II.15

For an $F_{\mathrm{p}}$-coalgebra $c: X \rightarrow F_{\mathrm{p}} X$, we define a PTS $M^{c}=$ $\left(X^{c}, \tau^{c}\right)$ and a set $\mathrm{Acc}^{c} \subseteq X^{c}$ of accepting states by: $X^{c}=X$, $\tau^{c}(x)=c_{1}(x)$ for each $x \in X^{c}$, and $\operatorname{Acc}^{c}=\{x \in X \mid$ $\left.c_{2}(x)=1\right\}$. Here we write $c(x)=\left(c_{1}(x), c_{2}(x)\right) \in \mathcal{D} X \times$ $\{0,1\}$ for every $x$.

Conversely, for a PTS $M=(X, \tau)$ and a set Acc $\subseteq X$, we define an $F_{\mathrm{p}}$-coalgebra $c^{M, \text { Acc }}: X \rightarrow F_{\mathrm{p}} X$ by $c^{M, \overline{\text { Acc }}}(x)=$ $(\tau(x), t)$ where $t$ is 1 if $x \in$ Acc and 0 otherwise.

These correspondences are indeed bijective. Analogously to Example III.6, we characterize reachability probabilities of a PTS as a coalgebraic least fixed-point property.

Proposition IV.2. We define an $F_{\mathrm{p}}$-modality $\sigma_{\mathrm{p}}: F_{\mathrm{p}}[0,1] \rightarrow$ $[0,1]$ over the truth-value domain $([0,1], \leq)$ as follows. Here $[0,1]$ is the unit interval and $\leq$ is the usual order on it.

$$
\sigma_{\mathrm{p}}(\delta, t)= \begin{cases}1 & (t=1) \\ \sum_{a \in \operatorname{supp}(\delta)} a \cdot \delta(a) & \text { (otherwise) } .\end{cases}
$$

Note here that $\delta \in \mathcal{D}[0,1]$ and $t \in\{0,1\}$.

Then $\sigma_{\mathrm{p}}$ satisfies Asm. III.7 and thus has least fixed points (in the sense of Def. III.5). The lfp property $\llbracket \mu \sigma_{\mathrm{p}} \rrbracket_{c}$ coincides

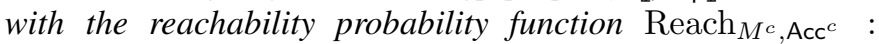
$X \rightarrow[0,1]$ of the corresponding PTS (Def. [I.9).
B. Known Variations: $\varepsilon$-Additive and $\alpha$-Multiplicative Ranking Supermartingales

The definition of ranking supermartingale that we have reviewed ( $\varepsilon$-additive ones in Def. II.11) is not an instance of our categorical notion (Def. III.10). Specifically, its value domain (the interval $[0, \infty]$ with a suitable $F_{\mathrm{p}}$-algebraic structure) fails to be corecursive. As a result, soundness of additive ranking supermartingale (Thm. II.12) cannot be directly proved using our categorical soundness theorem (Thm. III.12).

The following is an attempt to define a ranking domain for $\varepsilon$-additive supermartingales. Let us fix a real number $\varepsilon>0$ and define an $F_{\mathrm{p}}$-algebra $r_{\mathrm{p}, \varepsilon}^{\prime}: F_{\mathrm{p}}[0, \infty] \rightarrow[0, \infty]$, an arrow $q_{\mathrm{p}}^{\prime}:[0, \infty] \rightarrow[0,1]$ and a partial order $\Xi_{[0, \infty]}$ over $[0, \infty]$ as follows.

1) For each $(\psi, t) \in F_{\mathrm{p}}[0, \infty]=\mathcal{D}[0, \infty] \times\{0,1\}$,

$$
r_{\mathrm{p}, \varepsilon}^{\prime}(\psi, t)= \begin{cases}0 & (t=1) \\ \left(\sum_{a \in \operatorname{supp}(\psi)} a \cdot \psi(a)\right)+\varepsilon & \text { (otherwise) } .\end{cases}
$$

2) $q_{\mathrm{p}}^{\prime}(\infty)=0$ and $q_{\mathrm{p}}^{\prime}(a)=1$ if $a<\infty$.

3) $a \sqsubseteq_{[0, \infty]} b \stackrel{\text { def. }}{\Leftrightarrow} a \geq b$ (note the direction).

Proposition IV.3. In this setting, for each $c: X \rightarrow F_{\mathrm{p}} X$ and $b^{\prime}: X \rightarrow[0, \infty]$, we have the following.

a) $b^{\prime}$ is an $\varepsilon$-additive ranking supermartingale (Def. II.11) iff $b^{\prime}$ satisfies $b^{\prime} \sqsubseteq_{[0, \infty]} r_{\mathrm{p}, \varepsilon}^{\prime} \circ F_{\mathrm{p}} b^{\prime} \circ c$.

b) $b^{\prime}(x) \neq \infty$ iff $q_{\mathrm{p}}^{\prime} \circ b^{\prime}(x)=1$.

Therefore the triple $\left(r_{\mathrm{p}, \varepsilon}^{\prime}, q_{\mathrm{p}}^{\prime}, \sqsubseteq_{[0, \infty]}\right)$ is suited for accommodating $\varepsilon$-additive supermartingales in our categorical framework. Unfortunately it is not a ranking domain (Def. III.10).

Proposition IV.4. The triple $\left(r_{\mathrm{p}, \varepsilon}^{\prime}, q_{\mathrm{p}}^{\prime}, \sqsubseteq_{[0, \infty]}\right)$ introduced above satisfies the conditions of a ranking domain (Def. [III.10), except for Cond. 4

Example IV.5. We define a coalgebra $c: X \rightarrow F_{\mathrm{p}} X$ by: $X=\left\{x_{0}, x_{1}, x_{2}, x_{3}\right\}$, $c\left(x_{0}\right)=\left(\left[x_{1} \mapsto \frac{1}{2}, x_{2} \mapsto \frac{1}{2}\right], 0\right), c\left(x_{1}\right)=$ $\left(\left[x_{1} \mapsto \frac{1}{2}, x_{3} \mapsto \frac{1}{2}\right], 0\right), c\left(x_{2}\right)=\left(\left[x_{3} \mapsto\right.\right.$ $1], 0)$ and $c\left(x_{3}\right)=\left(\left[x_{3} \mapsto 1\right], 1\right)$. The corresponding PTS is depicted on the right.

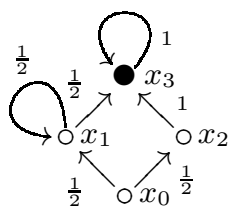

Let $\varepsilon>0$. For this coalgebra, we define arrows $b_{1}, b_{2}$ : $X \rightarrow[0, \infty]$ by: $b_{1}\left(x_{0}\right)=\frac{5}{2} \varepsilon, b_{1}\left(x_{1}\right)=2 \varepsilon, b_{1}\left(x_{2}\right)=\varepsilon$ and $b_{1}\left(x_{3}\right)=0$; and $b_{2}\left(x_{0}\right)=\infty, b_{2}\left(x_{1}\right)=\infty, b_{2}\left(x_{2}\right)=\varepsilon$ and $b_{2}\left(x_{3}\right)=0$. Both of these qualify as coalgebra-algebra homomorphisms from $c$ to $r_{\mathrm{p}, \varepsilon}^{\prime}$. Therefore $r_{\mathrm{p}, \varepsilon}^{\prime}$ is not corecursive.

It is well-known that $\varepsilon$-additive supermartingales (Def. II.11) witness positive almost-sure reachability [31], that is, the expected number of steps to accepting states is finite. This is a property strictly stronger than almost-sure reachability (see Example IV.6. It follows that $\varepsilon$-additive supermartingales are not complete against almost-sure reachability. 
Example IV.6. We define $c$ : $X \rightarrow F_{\mathrm{p}} X$ by $X=\{x\} \cup\left\{x_{i, j} \mid\right.$ $\left.i, j \in \mathbb{N}, 1 \leq i, 1 \leq j \leq 2^{i}\right\}$ and $c(x)=(\delta, 0)$, where $\delta\left(x_{i, j}\right)$ is $\frac{1}{2^{i}}$ if $j=0$ and 0 otherwise, $c\left(x_{i, j}\right)=\left(\left[x_{i, j+1} \mapsto 1\right], 0\right)$ for each $i$ and $j<2^{i}$, and $c\left(x_{i, 2^{i}}\right)=$ $\left(\left[x_{i, 2^{i}} \mapsto 1\right], 1\right)$. The corresponding

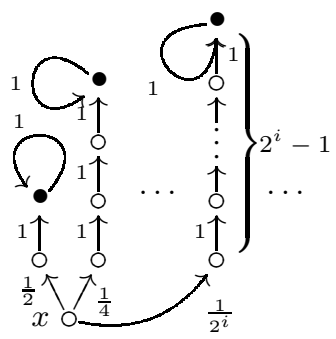

PTS $M^{c}$ and the set $A_{c c}^{c}$ of accepting states are shown on the right. This system when run from $x$ is clearly almostsure terminating; however the expected number of steps to accepting states is infinite.

Another known variation of ranking supermartingales is given by multiplicative ranking supermartingales [23]. Let $\alpha \in(0,1)$. A function $b: X \rightarrow[0, \infty]$ is an $\alpha$-multiplicative ranking supermartingale if we have

$$
\forall x \in X \backslash \text { Acc. } \sum_{x^{\prime} \in \operatorname{supp}(\tau(x))} \tau(x)\left(x^{\prime}\right) \cdot b\left(x^{\prime}\right) \leq \alpha \cdot b(x),
$$

and moreover there exists $\delta>0$ such that $b(x) \geq \delta$ for each $x \in X \backslash$ Acc. For this multiplicative variation, results analogous to Prop. IV.3 and Prop. IV.4 hold (see $\mathrm{G}$ in the appendix for the proofs).

\section{Distribution-Valued Ranking Functions}

Let us turn to possible instantiations of our categorical framework in the current probabilistic setting. The first uses $\mathcal{D} \mathbb{N}_{\infty}$, the set of distributions over extended natural numbers, as a ranking domain (instead of $[0, \infty]$ ). In what follows, given a probability distribution $\gamma$ over $\mathbb{N}_{\infty}$ and $a, b \in \mathbb{N}, \gamma([a, b])$ denotes $\sum_{i=a}^{b} \gamma(i)$ and $\gamma([a, \infty))$ denotes $\sum_{i=a}^{\infty} \varphi(i)$.

Proposition IV.7. Recall that $\mathbb{N}_{\infty}=\mathbb{N} \cup\{\infty\}$ and $\mathcal{D} \mathbb{N}_{\infty}$ collects all the distributions over $\mathbb{N}_{\infty}$. We define an $F_{\mathrm{p}}$-algebra $r_{\mathrm{p}}: F_{\mathrm{p}} \mathcal{D} \mathbb{N}_{\infty} \rightarrow \mathcal{D} \mathbb{N}_{\infty}$, a function $q_{\mathrm{p}}: \mathcal{D} \mathbb{N}_{\infty} \rightarrow[0,1]$ and $a$ partial order $\sqsubseteq \mathcal{D} \mathbb{N}_{\infty}$ over $\mathcal{D} \mathbb{N}_{\infty}$ as follows.

1) For each $(\Gamma, t) \in F_{\mathrm{p}} \mathcal{D} \mathbb{N}_{\infty}=\mathcal{D}^{2} \mathbb{N}_{\infty} \times\{0,1\}$,

$$
r_{\mathrm{p}}(\Gamma, t)(a)= \begin{cases}1 & (t=1, a=0) \\ 0 & (t=1, a>0 \text { or } t=0, a=0) \\ \sum_{\gamma \in \operatorname{supp}(\Gamma)} \Gamma(\gamma) \cdot \gamma(a-1) & (t=0, a>0) .\end{cases}
$$

2) $q_{\mathrm{p}}(\varphi)=\varphi([0, \infty))$.

3) $\varphi \sqsubseteq_{\mathcal{D N}_{\infty}} \varphi^{\prime} \stackrel{\text { def. }}{\Leftrightarrow} \forall a \in \mathbb{N} . \varphi([0, a]) \leq \varphi^{\prime}([0, a])$.

Then the triple $\left(r_{\mathrm{p}}, q_{\mathrm{p}}, \sqsubseteq \mathcal{D N _ { \infty }}\right)$ is a ranking domain. Moreover we have $q_{\mathrm{p}} \circ r_{\mathrm{p}}=\sigma_{\mathrm{p}} \circ F_{\mathrm{p}} q_{\mathrm{p}}$ (cf. (9) in Prop. III.18).

Intuitively, the value $b(x)([0, a]) \in[0,1]$ under-approximates the probability with which an accepting state is reached from $x$ within $a$ steps. The definition of $\sqsubseteq_{\mathcal{D N}}$, which is much like in probabilistic powerdomains (see e.g. [32]), also reflects this intuition. Here the Dirac distribution $\delta_{0}$ (resp. $\delta_{\infty}$ ) is the greatest (resp. least) element.

The definition of ranking arrow (Def. III.11) instantiates to the following - as one sees by straightforward calculationwhen we fix a ranking domain to be the one in Prop. IV.7
Definition IV.8 (distribution-valued ranking function). Let $M=(X, \tau)$ be a PTS and Acc $\subseteq X$. A distribution-valued ranking function is $b: X \rightarrow \mathcal{D N}_{\infty}$ such that:

$$
\sum_{x^{\prime} \in \operatorname{supp}(\tau)} \tau(x)\left(x^{\prime}\right) \cdot b\left(x^{\prime}\right)([0, a-1]) \geq b(x)([0, a])
$$

for each $x \in X \backslash$ Acc and $a \in \mathbb{N}_{\infty}$. Here we let $b\left(x^{\prime}\right)([0,-1])=0$.

By Thm. III.12 (soundness) we have the following: given a PTS $c: X \rightarrow F_{\mathrm{p}} X$ and an "assertion" $h: X \rightarrow[0,1]$, if there exists a distribution-valued ranking function $b: X \rightarrow \mathcal{D} \mathbb{N}_{\infty}$ such that $h \leq q_{\mathrm{p}} \circ b$, then we can conclude that $h \leq$ $\operatorname{Reach}_{M^{c}, \text { Acc }^{c} \text {. Here Reach }} M^{c}$, Acc $^{c}$ is given by reachability probabilities and coincides with $\llbracket \mu \sigma_{\mathrm{p}} \rrbracket_{c}$ (Prop. IV.2).

We note that quantitative verification is possible using distribution-valued ranking functions. For example an assertion $h: X \rightarrow[0,1]$ can be such that $h(x)=1 / 2$; by finding

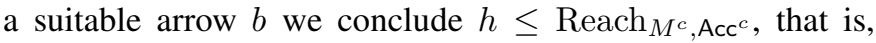
that the reachability probability from $x$ is at least $1 / 2 \sqrt[3]{3}$ Such a quantitative assertion cannot be verified using $\varepsilon$-additive supermartingales: as in Thm. II.12 they only witness the qualitative property of (positive) almost-sure reachability.

Example IV.9. We define a PTS $M=$ $(X, \tau)$ by $X=\left\{x_{0}, x_{1}, x_{2}\right\}, \tau\left(x_{0}\right)=$ $\left[x_{0} \mapsto \frac{1}{3}, x_{1} \mapsto \frac{1}{3}, x_{2} \mapsto \frac{1}{3}\right], \tau\left(x_{1}\right)=$ $\left[x_{1} \mapsto 1\right]$ and $\tau\left(x_{2}\right)=\left[x_{2} \mapsto 1\right]$. Let

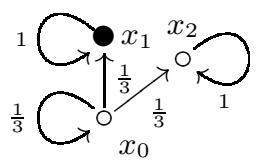
Acc $=\left\{x_{1}\right\}$. The function $b: X \rightarrow \mathcal{D N}_{\infty}$ defined by $b\left(x_{0}\right)=$ $\left[i \mapsto 1 / 3^{i+1}, \infty \mapsto 1 / 2\right], b\left(x_{1}\right)=[0 \mapsto 1]$ and $b\left(x_{2}\right)=[\infty \mapsto$ 1] is a distribution-valued ranking function. This allows us to conclude $\operatorname{Reach}_{M, \operatorname{Acc}}\left(x_{0}\right) \geq\left(1-\frac{1}{2}\right)=\frac{1}{2}$.

Finally we exhibit completeness of distribution-valued ranking functions. This is an immediate consequence of the categorical result (Prop. III.18) and Prop. IV.7

Proposition IV.10. For each $F_{\mathrm{p}}$-coalgebra $c: X \rightarrow F_{\mathrm{p}} X$, there exists a (categorical) ranking arrow $b: X \rightarrow \mathcal{D} \mathbb{N}_{\infty}$ such that $q_{\mathrm{p}} \circ b=\llbracket \mu \sigma_{\mathrm{p}} \rrbracket_{c}$.

Example IV.11. In Example IV.6, the function $b: X \rightarrow \mathcal{D N} \mathbb{N}_{\infty}$ defined by

$$
b(x)(n)=\left\{\begin{array}{ll}
1 / 2^{i} & \left(n=2^{i}, i>0\right) \\
0 & (\text { otherwise })
\end{array} \text { and } b\left(x_{i, j}\right)=\delta_{2^{i}-j}\right.
$$

is a distribution-valued ranking function (here $\delta_{2^{i}-j}$ denotes a Dirac distribution). We have $b(x)([0, \infty))=1$ and thus successfully verify almost-sure reachability from $x$.

In IV-B we have argued that $\varepsilon$-additive ranking supermartingales (Def. II.11) is not an instance of our categorical ranking arrow. It is nevertheless possible to prove its soundness (Thm. II.5) using the categorical framework-specifically by showing that an $\varepsilon$-additive ranking supermartingale gives rise to a distribution-valued ranking function (Def. IV.8). Details are found in $₫$ in the appendix.

\footnotetext{
${ }^{3}$ The problem solved here is more precisely the threshold reachability checking problem; and ranking function-based proof methods for the problem would be viable options especially when the state space $X$ is infinite.
} 
Similarly our framework can prove soundness of $\alpha$ multiplicative ranking supermartingales (see $\oint$ J for the details).

\section{D. $\gamma$-Scaled Non-Counting Ranking Supermartingales}

The notion of distribution-valued ranking function exhibits pleasant properties like completeness and quantitative assertion checking. A major drawback, however, is the complexity of its value domain $\mathcal{D} \mathbb{N}_{\infty}$.

In many realistic verification scenarios a ranking function/supermartingale $b$ would be synthesized as follows: the function $b$ is expressed in a predetermined template $b_{\vec{p}}$ (such as polynomials up-to a certain degree) in which some parameters $\vec{p}$ occur; the requirements on $b_{\vec{p}}$ translate to constraints on $\vec{p}$; and one relies on some optimization solver (for SAT, LP, $\mathrm{SDP}$, etc.) to solve the constraints. It significantly increases the complexity of the workflow if the value $b(x)$ is a distribution in $\mathcal{D} \mathbb{N}_{\infty}$ instead of an (extended) real number in $[0, \infty]$.

Here we present another probabilistic instantiation of the categorical framework. It takes values in the unit interval $[0,1]$.

Proposition IV.12. We fix a real number $\gamma \in[0,1)$. We define an algebra $r_{\mathrm{nc}, \gamma}: F_{\mathrm{p}}[0,1] \rightarrow[0,1]$ (here nc stands for "noncounting”) as follows.

$$
r_{\mathrm{nc}, \gamma}(\varphi, t)= \begin{cases}1 & (t=1) \\ \gamma \cdot \sum_{a \in \operatorname{supp}(\varphi)} a \cdot \varphi(a) & (\text { otherwise }) .\end{cases}
$$

We further define $q_{\mathrm{nc}}:[0,1] \rightarrow[0,1]$ by $q_{\mathrm{nc}}(a)=a$. Then $\left(r_{\mathrm{nc}, \gamma}, q_{\mathrm{nc}}, \leq\right)$, where $\leq$ is the usual on $[0,1]$, is a ranking domain with respect to the modality $\sigma_{\mathrm{p}}$ (Prop. IV.2).

Thus it makes sense to consider ranking arrows (Def. III.11) with respect to the ranking domain $\left(r_{\mathrm{nc}, \gamma}, q_{\mathrm{nc}}, \leq\right)$. By straightforward calculation, their definition unravels as follows.

Definition IV.13 ( $\gamma$-scaled non-counting ranking supermartingale). Let $M=(X, \tau)$ be a PTS and Acc $\subseteq X$. We fix a real number $\gamma$ such that $0 \leq \gamma<1$. A $\gamma$-scaled non-counting ranking supermartingale is a function $b: X \rightarrow[0,1]$ such that for each $x \in X \backslash$ Acc we have:

$$
\gamma \cdot \sum_{x^{\prime} \in X} \tau(x)\left(x^{\prime}\right) \cdot b\left(x^{\prime}\right) \geq b(x) .
$$

This notion of supermartingale seems new. The intuition is that $b(x)$ is a lower bound for the reachability probability. Note that, unlike for the other variations of supermartingales in this section (where we over-approximate the number of steps), reachability probabilities should be under-approximated.

We obtain the following soundness result as an instance of Thm. III.12, Here a non-counting ranking supermartingale $b$ itself gives lower bounds for reachability probabilities since $q_{\mathrm{nc}}:[0,1] \rightarrow[0,1]$ is the identity map.

Corollary IV.14. Let $M=(X, \tau)$ be a PTS and $b: X \rightarrow$ $[0,1]$ be a $\gamma$-scaled non-counting ranking supermartingale. Then we have $\operatorname{Reach}_{\mathrm{Acc}}(x) \geq b(x)$.

Example IV.15. Consider the PTS $M$ and Acc in Example IV.9. For each $\gamma \in[0,1)$, we define $b_{\gamma}: X \rightarrow[0,1]$ by $b_{\gamma}\left(x_{0}\right)=\frac{\gamma}{3-\gamma}, b_{\gamma}\left(x_{1}\right)=1$ and $b_{\gamma}\left(x_{2}\right)=0$. Then $b_{\gamma}$ is seen to be a $\gamma$-scaled non-counting ranking supermartingale. By Cor. IV.14 we have $\operatorname{Reach}_{\text {Acc }}\left(x_{0}\right) \geq \frac{\gamma}{3-\gamma}$; as this holds for any $\gamma \in[0,1)$, by letting $\gamma \rightarrow 1$, we conclude $\operatorname{Reach}_{\text {Acc }}\left(x_{0}\right) \geq \frac{1}{2}$.

We note that in general a scaling factor $\gamma \in[0,1)$ results in suboptimality of under-approximation of reachability properties: in the last example $b_{\gamma}\left(x_{0}\right)=\frac{\gamma}{3-\gamma}$ is smaller than the reachability probability $1 / 2$. Such suboptimality is an issue especially when we aim at qualitative verification of almost-sure reachability. In the last example we exercised an asymptotic argument in which we think of $\gamma$ as a free variable and take the limit under $\gamma \rightarrow 1$. This strategy can be employed for almost-sure reachability checking.

Finally, the following example demonstrates that application of non-counting supermartingales is not limited to positive almost-sure reachability.

Example IV.16. We define a PTS $M=(X, \tau)$ and Acc $\subseteq X$ as in Example IV.6. For each $\gamma$, if we define $b_{\gamma}: X \rightarrow[0,1]$ by $b_{\gamma}(x)=\sum_{i=1}^{\infty} \frac{\gamma^{2^{i}}}{2^{i}}$ and $b_{\gamma}\left(x_{i, j}\right)=\gamma^{2^{i}-j}$ for each $i$ and $j$, then it is a $\gamma$-scaled non-counting supermartingale. Hence we have $\operatorname{Reach}_{M, \operatorname{Acc}}(x) \geq \lim _{\gamma \rightarrow 1} b_{\gamma}(x)=1$.

Let us summarize the section. We presented four variations of ranking supermartingales: $\varepsilon$-additive ones, $\alpha$-multiplicative ones, distribution-valued ranking functions and $\gamma$-scaled noncounting ranking supermartingales. The former two are known in the literature while the latter two seem to be new. The known notions are not instances of our generic definition, but their soundness can be derived via our generic theory (see the end of $₫ \mathrm{IV}-\mathrm{C}$ ). Among the (seemingly) new notions, distribution-valued ranking functions enjoy nice properties like completeness (Prop. IV.10) and support of quantitative reasoning (see Example IV.9 - at the cost of their complexity (they take as values distributions in $\mathcal{D} \mathbb{N}_{\infty}$ ). Non-counting ranking supermartingales (whose values are simply real numbers) are advantageous in quantitative reasoning (see Example IV.15) and non-positive almost-sure termination, but the scaling factor $\gamma$ in it leads to suboptimal approximation. Typically one needs to rely on asymptotic arguments to obtain sharp bounds.

\section{COnClusions And Future Work}

We have given a categorical account for liveness checking: we identify the essence of ranking function-based proof methods as the combination of corecursive algebras (as value domains) and lax homomorphisms; and for our notion of ranking arrow a soundness theorem has been presented. Our leading examples have been two-player games and probabilistic transition systems; in the course of studying them we were led to (seemingly) new variations of ranking martingales.

Besides the concrete examples of "ranking functions" in this paper, we wish to derive yet other concrete examples from our categorical modeling, so that they provide novel proof methods for various liveness properties. A possible direction towards this goal is discussed in $₫ \mathrm{~L}$ in the appendix, motivated by categorical closure properties of corecursive algebras. Some abstract categorical questions remain open, too, such as 
characterization of the non-well-founded part-that represents failure of liveness properties - of a corecursive algebra. We are also interested in the relationship between these (expected) results/examples and productivity for coinductive datatypes in functional programming [35]. Intuitively, the latter is a property that any finite prefix of a coinductively defined data is obtained in finite time.

We have used two-player games (systems with angelic and demonic transitions) and PTSs (systems with probabilistic transitions) as leading examples. A natural direction of future work is to consider stochastic games, which involve angelic, demonic and probabilistic transitions [36].

In this paper we have focused on least fixed-point properties. Extension to nested fixed-point specifications-persistence, recurrence, and general fixed-point formulas-is important future work. There we will need to categorically axiomatize progress measures for parity games ([37]; see also [38]). Possibly relevant to this direction is our recent coalgebraic modeling of Büchi and parity acceptance conditions [39].

Practical implications of the proposed framework (and concrete "ranking functions" derived thereby) shall be investigated, too. We are especially interested in cyber-physical applications in which state spaces are inherently infinite but often allow succinct symbolic presentations (e.g. by polynomials). The work closely related to this direction is [23].

A categorical account on martingales is also found in recent [40], where a connection between two classic resultsKolmogorov's extension theorem and Doob's martingale convergence theorem-is established in categorical terms. The relationship between this work and ours shall be pursued, possibly centered around the notion of final sequence.

\section{ACKNOWLEDGMENT}

Thanks are due to Eugenia Sironi and anonymous referees for their useful comments. The authors are supported by ERATO HASUO Metamathematics for Systems Design Project (No. JPMJER1603), JST, and Grants-in-Aid No. 15KT0012 \& 15K11984, JSPS. Natsuki Urabe is supported by Grant-inAid for JSPS Fellows (No. 16J08157).

\section{REFERENCES}

[1] V. Schuppan and A. Biere, "Liveness checking as safety checking for infinite state spaces," Electr. Notes Theor. Comput. Sci., vol. 149, no. 1, pp. 79-96, 2006. [Online]. Available: http://dx.doi.org/10.1016/j.entcs.2005.11.018

[2] K. Claessen and N. Sörensson, "A liveness checking algorithm that counts," in Formal Methods in Computer-Aided Design, FMCAD 2012, Cambridge, UK, October 22-25, 2012, G. Cabodi and S. Singh, Eds. IEEE, 2012, pp. 52-59. [Online]. Available: http://ieeexplore.ieee.org/xpl/articleDetails.jsp?arnumber=6462555

[3] P. K. Nalla, R. K. Gajavelly, H. Mony, J. Baumgartner, and R. Kanzelman, "Effective liveness verification using a transformationbased framework," in 2014 27th International Conference on VLSI Design and 2014 13th International Conference on Embedded Systems, Mumbai, India, January 5-9, 2014. IEEE Computer Society, 2014, pp. 74-79. [Online]. Available: http://dx.doi.org/10.1109/VLSID.2014.20

[4] R. W. Floyd, "Assigning meanings to programs," Proceedings of Symposium on Applied Mathematics, vol. 19, pp. 19-32, 1967.
[5] A. Chakarov and S. Sankaranarayanan, "Probabilistic program analysis with martingales," in Computer Aided Verification - 25th International Conference, CAV 2013, Saint Petersburg, Russia, July 13-19, 2013. Proceedings, ser. Lecture Notes in Computer Science, N. Sharygina and H. Veith, Eds., vol. 8044. Springer, 2013, pp. 511-526. [Online]. Available: http://dx.doi.org/10.1007/978-3-642-39799-8_34

[6] L. M. F. Fioriti and H. Hermanns, "Probabilistic termination: Soundness, completeness, and compositionality," in Proceedings of the 42nd Annual ACM SIGPLAN-SIGACT Symposium on Principles of Programming Languages, POPL 2015, Mumbai, India, January 15-17, 2015, S. K. Rajamani and D. Walker, Eds. ACM, 2015, pp. 489-501. [Online]. Available: http://doi.acm.org/10.1145/2676726.2677001

[7] B. Jacobs, Introduction to Coalgebra: Towards Mathematics of States and Observation, ser. Cambridge Tracts in Theoretical Computer Science. Cambridge University Press, 2016.

[8] J. J. M. M. Rutten, "Universal coalgebra: a theory of systems," Theor Comp. Sci., vol. 249, pp. 3-80, 2000.

[9] A. Sokolova, "Probabilistic systems coalgebraically: A survey," Theor. Comput. Sci., vol. 412, no. 38, pp. 5095-5110, 2011. [Online]. Available: http://dx.doi.org/10.1016/j.tcs.2011.05.008

[10] P. Aczel and N. P. Mendler, "A final coalgebra theorem," in Category Theory and Computer Science, Manchester, UK, September 5-8, 1989, Proceedings, ser. Lecture Notes in Computer Science, D. H. Pitt, D. E. Rydeheard, P. Dybjer, A. M. Pitts, and A. Poigné, Eds., vol. 389. Springer, 1989, pp. 357-365.

[11] I. Hasuo, "Generic forward and backward simulations," in CONCUR, ser. Lecture Notes in Computer Science, C. Baier and H. Hermanns, Eds., vol. 4137. Springer, 2006, pp. 406-420.

[12] N. Urabe and I. Hasuo, "Coalgebraic infinite traces and Kleisli simulations," in Algebra and Coalgebra in Computer Science - 6th International Conference, CALCO 2015, Nijmegen, Netherlands, June 24-26, 2015. Proceedings, 2015.

[13] V. Capretta, T. Uustalu, and V. Vene, "Corecursive algebras: A study of general structured corecursion," in Formal Methods: Foundations and Applications, 12th Brazilian Symposium on Formal Methods, SBMF 2009, Gramado, Brazil, August 19-21, 2009, Revised Selected Papers, ser. Lecture Notes in Computer Science, M. V. M. Oliveira and J. Woodcock, Eds., vol. 5902. Springer, 2009, pp. 84-100. [Online]. Available: http://dx.doi.org/10.1007/978-3-642-10452-7_7

[14] M. B. Smyth and G. D. Plotkin, "The category theoretic solution of recursive domain equations," SIAM Journ. Comput., vol. 11, pp. 761783, 1982

[15] P. J. Freyd, "Algebraically complete categories," in Como Conference on Category Theory, ser. Lect. Notes Math., A. Carboni, M. C. Pedicchio, and G. Rosolini, Eds., no. 1488. Springer, Berlin, 1991, pp. 95-104.

[16] I. Hasuo, B. Jacobs, and A. Sokolova, "Generic trace semantics via coinduction," Logical Methods in Computer Science, vol. 3, no. 4, 2007.

[17] I. Hasuo, "Generic weakest precondition semantics from monads enriched with order," Theor. Comput. Sci., vol. 604, pp. 2-29, 2015. [Online]. Available: http://dx.doi.org/10.1016/j.tcs.2015.03.047

[18] W. Hino, H. Kobayashi, I. Hasuo, and B. Jacobs, "Healthiness from duality," in Proceedings of the 31st Annual ACM/IEEE Symposium on Logic in Computer Science, LICS '16, New York, NY, USA, July 5-8, 2016, M. Grohe, E. Koskinen, and N. Shankar, Eds. ACM, 2016, pp. 682-691. [Online]. Available: http://doi.acm.org/10.1145/2933575.2935319

[19] L. Schröder and D. Pattinson, "PSPACE bounds for rank-1 modal logics," ACM Trans. Comput. Log., vol. 10, no. 2, 2009.

[20] C. Urban and A. Miné, "An abstract domain to infer ordinalvalued ranking functions," in Programming Languages and Systems - 23rd European Symposium on Programming, ESOP 2014, Held as Part of the European Joint Conferences on Theory and Practice of Software, ETAPS 2014, Grenoble, France, April 5-13, 2014 Proceedings, ser. Lecture Notes in Computer Science, Z. Shao, Ed., vol. 8410. Springer, 2014, pp. 412-431. [Online]. Available: http://dx.doi.org/10.1007/978-3-642-54833-8_22

[21] Z. Manna and A. Pnueli, "Adequate proof principles for invariance and liveness properties of concurrent programs," Sci. Comput. Program., vol. 4, no. 3, pp. 257-289, 1984. [Online]. Available: http://dx.doi.org/10.1016/0167-6423(84)90003-0

[22] E. Grädel and I. Walukiewicz, "Positional determinacy of games with infinitely many priorities," Logical Methods in Computer Science, vol. 2, no. 4, 2006. [Online]. Available: http://dx.doi.org/10.2168/LMCS-2(4:6)2006 
[23] A. N. Chakarov, "Deductive verification of infinite-state stochastic systems using martingales," Ph.D. dissertation, University of Colorado, 2016.

[24] S. Mac Lane, Categories for the working mathematician, 2nd ed. Springer-Verlag New York, 1998, vol. 5.

[25] G. Osius, "Categorical set theory: A characterization of the category of sets," Journal of Pure and Applied Algebra, vol. 4, no. 1, pp. 79 - 119, 1974. [Online]. Available: http://www.sciencedirect.com/science/article/pii/0022404974900322

[26] P. Taylor, Practical foundations of mathematics, ser. Cambridge studies in advanced mathematics. Cambridge, New York (N. Y.), Melbourne: Cambridge University Press, 1999. [Online]. Available: http://opac.inria.fr/record=b1095522

[27] J. Adámek, S. Milius, L. S. Moss, and L. Sousa, "Well-pointed coalgebras," Logical Methods in Computer Science, vol. 9, no. 3, 2013. [Online]. Available: http://dx.doi.org/10.2168/LMCS-9(3:2)2013

[28] P. B. Levy, "Final coalgebras from corecursive algebras," in 6th Conference on Algebra and Coalgebra in Computer Science, CALCO 2015, June 24-26, 2015, Nijmegen, The Netherlands, ser. LIPIcs, L. S. Moss and P. Sobocinski, Eds., vol. 35. Schloss Dagstuhl - Leibniz-Zentrum fuer Informatik, 2015, pp. 221-237. [Online]. Available: http://dx.doi.org/10.4230/LIPIcs.CALCO.2015.221

[29] P. Cousot and R. Cousot, "Constructive versions of Tarski's fixed point theorems," Pacific Journal of Mathematics, vol. 82, no. 1, pp. 43-57, 1979.

[30] G. Plotkin, "Notes on domains (pisa notes)," 1983, available at http://www.dcs.ed.ac.uk/home/gdp/publications/.

[31] O. Bournez and F. Garnier, "Proving positive almost-sure termination," in Term Rewriting and Applications, 16th International Conference, RTA 2005, Nara, Japan, April 19-21, 2005, Proceedings, ser. Lecture Notes in Computer Science, J. Giesl, Ed., vol. 3467. Springer, 2005, pp. 323-337. [Online]. Available: http://dx.doi.org/10.1007/978-3-540-32033-3_24

[32] N. Saheb-Djahromi, "Cpo's of measures for nondeterminism," Theor Comput. Sci., vol. 12, pp. 19-37, 1980. [Online]. Available: http://dx.doi.org/10.1016/0304-3975(80)90003-1

[33] J. Adámek, M. Haddadi, and S. Milius, "Corecursive algebras, corecursive monads and bloom monads," Logical Methods in Computer Science, vol. 10, no. 3, 2014. [Online]. Available: http://dx.doi.org/10.2168/LMCS-10(3:19)2014

[34] V. Capretta, T. Uustalu, and V. Vene, "Recursive coalgebras from comonads," Inf. Comput., vol. 204, no. 4, pp. 437-468, 2006. [Online]. Available: http://dx.doi.org/10.1016/j.ic.2005.08.005

[35] A. Abel and B. Pientka, "Well-founded recursion with copatterns and sized types," J. Funct. Program., vol. 26, p. e2, 2016. [Online]. Available: http://dx.doi.org/10.1017/S0956796816000022

[36] L. S. Shapley, "Stochastic games," Proceedings of the National Academy of Sciences, vol. 39, no. 10, pp. 1095-1100, 1953. [Online]. Available: http://www.pnas.org/content/39/10/1095.short

[37] M. Jurdzinski, "Small progress measures for solving parity games," in STACS, ser. Lecture Notes in Computer Science, H. Reichel and S. Tison, Eds., vol. 1770. Springer, 2000, pp. 290-301.

[38] I. Hasuo, S. Shimizu, and C. Cîrstea, "Lattice-theoretic progress measures and coalgebraic model checking," in Proceedings of the 43rd Annual ACM SIGPLAN-SIGACT Symposium on Principles of Programming Languages, POPL 2016, St. Petersburg, FL, USA, January 20 - 22, 2016, R. Bodik and R. Majumdar, Eds. ACM, 2016, pp. 718 732. [Online]. Available: http://doi.acm.org/10.1145/2837614.2837673

[39] N. Urabe, S. Shimizu, and I. Hasuo, "Coalgebraic trace semantics for Büchi and parity automata," in 27th International Conference on Concurrency Theory, CONCUR 2016, August 2326, 2016, Québec City, Canada, ser. LIPIcs, J. Desharnais and R. Jagadeesan, Eds., vol. 59. Schloss Dagstuhl - LeibnizZentrum fuer Informatik, 2016, pp. 24:1-24:15. [Online]. Available: http://dx.doi.org/10.4230/LIPIcs.CONCUR.2016.24

[40] D. Kozen, "Kolmogorov extension, martingale convergence, and compositionality of processes," in Proc. 31st ACM-IEEE Symp. Logic in Computer Science (LICS 2016), N. Shankar, Ed. New York City: ACM, July 2016, pp. 692-699. 


\section{APPENDIX}

\section{A. Formal Discussions for Two-Player Games}

Definition A.1 $\left(\sigma_{\mathrm{g}}\right)$. Let $(\{0,1\}, \leq)$ be a truth-value domain where $\leq$ denotes the usual order. We define an $F_{\mathrm{g}}$-modality $\sigma_{\mathrm{g}}: F_{\mathrm{g}}\{0,1\} \rightarrow\{0,1\}$ by

$$
\sigma_{\mathrm{g}}(\Gamma, t)= \begin{cases}1 & (t=1) \\ \max _{A \in \Gamma} \min _{a \in A} a & \text { (otherwise) } .\end{cases}
$$

Proposition A.2. We define an $F_{\mathrm{g}}$-modality $\sigma_{\mathrm{g}}: F_{\mathrm{g}}\{0,1\} \rightarrow$ $\{0,1\}$ as in Def. A.1

1) The modality $\sigma_{\mathrm{g}}$ has least fixed points, and for each coalgebra $c: X \rightarrow F_{\mathrm{g}} X$, the corresponding least fixedpoint property $\llbracket \mu \sigma_{\mathrm{g}} \rrbracket_{c}: X \rightarrow\{0,1\}$ is given as follows:

$$
\llbracket \mu \sigma_{\mathrm{g}} \rrbracket_{c}(x)= \begin{cases}1 & \left(x \in \text { Reach }_{G^{c}, \text { Acc }^{c}}\right) \\ 0 & (\text { otherwise }) .\end{cases}
$$

2) For a game structure $G=\left(X_{\max }, X_{\min }, \tau\right)$ and a set Acc $\subseteq X_{\max }$, we have:

$$
\llbracket \mu \sigma_{\mathrm{g}} \rrbracket_{c^{G, \mathrm{Acc}}}(x)= \begin{cases}1 & \left(x \in \text { Reach }_{G, \mathrm{Acc}}\right) \\ 0 & (\text { otherwise }) .\end{cases}
$$

Proof. We first prove (1). We define $f: X \rightarrow\{0,1\}$ by

$$
f(x)= \begin{cases}1 & \left(x \in \text { Reach }_{G^{c}, \text { Acc }^{c}}\right) \\ 0 & \text { (otherwise }) .\end{cases}
$$

By definition of the least fixed-point property, it suffices to show that $f$ is the least fixed point of the function $\Phi_{c, \sigma_{\mathrm{g}}}$ : $\{0,1\}^{X} \rightarrow\{0,1\}^{X}$ in Def. III.2

We first show that $f$ is a fixed point of $\Phi_{c, \sigma_{\mathrm{g}}}$. For each $x \in X$, we have:

$$
\begin{aligned}
& \Phi_{c, \sigma_{\mathrm{g}}}(f)(x)=1 \\
& \left.\Leftrightarrow\left(\sigma_{\mathrm{g}} \circ F_{\mathrm{g}} f \circ c\right)(x)=1 \quad \text { (by def. of } \Phi_{c, \sigma_{\mathrm{g}}}\right) \\
& \Leftrightarrow c_{2}(x)=1, \text { or } \exists A \in c_{1}(x) . \forall x^{\prime} \in A . f\left(x^{\prime}\right)=1
\end{aligned}
$$$$
\text { (by def. of } \sigma_{\mathrm{g}} \text { and } F_{\mathrm{g}} \text { ) }
$$$$
\Leftrightarrow c_{2}(x)=1 \text {, or }
$$$$
\exists A \in c_{1}(x) . \forall x^{\prime} \in A .
$$

$\exists \alpha^{\prime}$ : a strategy of max. $\forall \beta^{\prime}$ : a strategy of min.

$$
\rho^{\alpha^{\prime}, \beta^{\prime}, x^{\prime}} \text { is winning for max (by def. of } f \text { ) }
$$$$
\Leftrightarrow c_{2}(x)=1 \text {, or }
$$$$
\exists A \in c_{1}(x) .
$$$$
\exists\left(\alpha_{x^{\prime}}^{\prime}\right)_{x^{\prime} \in A} \text { : a family of strategies of max. }
$$$$
\forall x^{\prime} \in A . \forall \beta^{\prime} \text { : a strategy of min. }
$$$$
\rho^{\alpha_{x^{\prime}}^{\prime}, \beta^{\prime}, x^{\prime}} \text { is winning for max }
$$

$\Leftrightarrow x \in \mathrm{Acc}^{c}$, or

$\exists y \in X_{\text {min }}^{c}$ s.t. $(x, y) \in \tau^{c}$.

$\exists\left(\alpha_{x^{\prime}}^{\prime}\right)_{x^{\prime} \in X_{\text {max }}^{c}}$ s.t. $\left(y, x^{\prime}\right) \in \tau^{c}:$ a family of strategies of max.

$\forall x^{\prime} \in X_{\max }^{c}$ s.t. $\left(y, x^{\prime}\right) \in \tau^{c} . \forall \beta^{\prime}:$ a strategy of min. $\rho^{\alpha_{x^{\prime}}^{\prime}, \beta^{\prime}, x^{\prime}}$ is winning for max (by def. of $G^{c}$ and $\mathrm{Acc}^{c}$ )

$\Leftrightarrow \exists \alpha$ : a strategy of max. $\forall \beta$ : a strategy of min.

$$
\begin{array}{rr}
\rho^{\alpha, \beta, x} \text { is winning for max } & \text { (by def. of } \rho^{\alpha, \beta, x} \text { ) } \\
\Leftrightarrow f(x)=1 & \text { (by def. of } f \text { ). }
\end{array}
$$

Hence $f$ is a fixed point of $\Phi_{c, \sigma_{g}}$.

It remains to show that $f: X \rightarrow\{0,1\}$ is the least fixed point with respect to the pointwise extension of $\leq$. Let $f^{\prime}$ : $X \rightarrow\{0,1\}$ be a fixed point of $\Phi_{c, \sigma_{g}}$. To prove $f \leq f^{\prime}$, it suffices to prove that $f^{\prime}(x)=0$ implies $f(x)=0$ for each $x \in X$.

For each $x^{\prime} \in X$, we have:

$$
\begin{aligned}
& f^{\prime}\left(x^{\prime}\right)=0 \\
& \Leftrightarrow \Phi_{c, \sigma_{\mathrm{g}}}\left(f^{\prime}\right)\left(x^{\prime}\right)=0 \quad\left(f^{\prime} \text { is a fixed point of } \Phi_{c, \sigma_{\mathrm{g}}}\right) \\
& \Leftrightarrow\left(\sigma_{\mathrm{g}} \circ F_{\mathrm{g}} f^{\prime} \circ c\right)\left(x^{\prime}\right)=0 \\
& \Leftrightarrow c_{2}\left(x^{\prime}\right)=0, \text { and } \forall A \in c_{1}\left(x^{\prime}\right) . \exists x^{\prime \prime} \in A . f^{\prime}\left(x^{\prime \prime}\right)=0
\end{aligned}
$$

(by def. of $\sigma_{\mathrm{g}}$ and $F_{\mathrm{g}}$ )

$$
\begin{aligned}
& \Leftrightarrow x^{\prime} \notin \mathrm{Acc}^{c}, \text { and } \\
& \forall y \in X_{\min }^{c} \text { s.t. }\left(x^{\prime}, y\right) \in \tau^{c} . \\
& \quad \exists x^{\prime \prime} \in X_{\max }^{c} \text { s.t. }\left(y, x^{\prime \prime}\right) \in \tau^{c} \cdot f^{\prime}\left(x^{\prime \prime}\right)=0
\end{aligned}
$$

(by def. of $G^{c}$ and $\mathrm{Acc}^{c}$ ).

This means that if $f^{\prime}\left(x^{\prime}\right)=0$, then $c_{2}\left(x^{\prime}\right)=0$ and for each $A \in c_{1}\left(x^{\prime}\right)$ there exists $x^{\prime \prime} \in A$ such that $f^{\prime}\left(x^{\prime \prime}\right)=0$. Hence for each $x \in X$ such that $f^{\prime}(x)=0$, we can define a strategy $\beta_{x}$ of the player min so that for each strategy $\alpha$ of the player $\max$, the resulting run $\rho^{\alpha, \beta_{x}, x}$ from $x$ is not winning for max.

Therefore by the definition of $f$, we have $f(x)=0$. This concludes the proof.

The item (2) is proved in a similar way.

\section{1) Details for Example III.9.}

Proposition A.3. The modality $\sigma_{\mathrm{g}}: F_{\mathrm{g}}\{0,1\} \rightarrow\{0,1\}$ in Def. A.1 satisfies Asm. III.7

Proof. It is easy to see that Cond. 11 is satisfied. By monotonicity of the functions max and $\min$ that are used in $\sigma_{\mathrm{g}}$, Cond. 2 is satisfied.

\section{2) Details of Example III.15.}

Proposition A.4. We define an $F_{\mathrm{g}}$-modality $\sigma_{\mathrm{g}}$ as in Def. A.1.

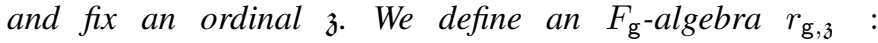
$F_{\mathrm{g}} \mathrm{Ord}_{\leq \mathfrak{z}} \rightarrow$ Ord $_{\leq \mathfrak{z}}$, a function $q_{\mathrm{g}, \mathfrak{z}}: F_{\mathrm{g}} \operatorname{Ord}_{\leq \mathfrak{z}} \rightarrow\{0,1\}$ and a partial order $\sqsubseteq$ Ord over $\operatorname{Ord}_{\leq_{\mathfrak{z}}}$ as follows.

1) $\operatorname{Ord}_{\leq \mathfrak{z}}=\{\mathfrak{a} \mid \mathfrak{a}$ is an ordinal s.t. $\mathfrak{a} \leq \mathfrak{z}\}$, and $r_{\mathrm{g}, \mathfrak{z}}$ : $F_{\mathrm{g}} \mathrm{Ord}_{\leq \mathfrak{z}} \rightarrow \mathrm{Ord}_{\leq \mathfrak{z}}$ is defined by

$$
r_{\mathrm{g}, \mathfrak{z}}(\Gamma, t)= \begin{cases}0 & (t=1) \\ \min _{A \in \Gamma} \sup _{\mathfrak{a} \in A}(\mathfrak{a} \widehat{+} 1) & (\text { otherwise }) .\end{cases}
$$

Here $\mathfrak{a} \widehat{+} 1$ denotes $\min \{\mathfrak{a}+1, \mathfrak{z}\}$.

2) $q_{\mathrm{g}, \mathfrak{z}}: \operatorname{Ord}_{\leq \mathfrak{z}} \rightarrow\{0,1\}$ is defined by

$$
q_{\mathrm{g}, \mathfrak{z}}(\mathfrak{a})= \begin{cases}0 & (\mathfrak{a}=\mathfrak{z}) \\ 1 & (\text { otherwise }) .\end{cases}
$$


3) For $\mathfrak{a}, \mathfrak{b} \in \operatorname{Ord}_{\leq \mathfrak{z}}$, $\mathfrak{a} \sqsubseteq$ Ord $\mathfrak{b} \stackrel{\text { def. }}{\Leftrightarrow} \mathfrak{a} \geq \mathfrak{b}$ (note the directions).

Then the triple $\left(r_{\mathrm{g}, \mathfrak{z}}, q_{\mathrm{g}, \mathfrak{z}}\right.$, Ord $)$ is a ranking domain.

The most difficult part of the proof is to prove that $r$ is a corecursive algebra (Cond. 4 in Def. III.10). We prove it separately. To this end, we first prove the following sublemma.

Lemma A.5. The algebra $r_{\mathrm{g}, \mathfrak{z}}: F_{\mathrm{g}} \operatorname{Ord}_{\leq \mathfrak{z}} \rightarrow \operatorname{Ord}_{\leq \mathfrak{z}}$ satisfies Cond. 2 in Def. III.10.

Proof. It is easy to see that Cond. $2 \mathrm{a}$ is satisfied.

We prove that Cond. $2 \mathrm{~b}$ is satisfied. Let $b_{1}, b_{2}: X \rightarrow$ $\operatorname{Ord}_{\leq \mathfrak{z}}$ and assume that $b_{1} \sqsubseteq$ Ord $b_{2}$. Let $x \in X$. As 0 is the greatest element in $\left(\operatorname{Ord}_{\leq \mathfrak{z}}, \sqsubseteq\right.$ Ord $)$, if $\Phi_{c, r_{\mathfrak{g}, \mathfrak{z}}}\left(b_{2}\right)(x)=0$ then we have $\Phi_{c, r_{\mathrm{g}, \mathfrak{z}}}\left(b_{1}\right)(x) \sqsubseteq$ Ord $\Phi_{c, r_{\mathrm{g}, \mathfrak{z}}}\left(b_{2}\right)(x)$.

Assume that $\Phi_{c, r_{\mathrm{g}, \mathfrak{z}}}\left(b_{2}\right)(x)=n \in \operatorname{Ord}_{\leq \mathfrak{z}} \backslash\{0\}$. Let $c(x)=(\Gamma, t) \in F_{\mathrm{g}} X=\mathcal{P}^{2} X \times\{0,1\}$. Then by definition of $r_{\mathrm{g}, \mathfrak{z}}$ and $F_{\mathrm{g}}, t=0$ and moreover for all $A \in \Gamma$ there exists $x \in A$ such that $b_{2}(x) \geq n$. As $b_{1} \sqsubseteq$ Ord $b_{2}, b_{2}(x) \geq n$ implies $b_{1}(x) \geq n$. Hence we have $r_{\mathrm{g}, \mathfrak{z}} \circ F_{\mathrm{g}} b_{1}(\Gamma, t) \geq n$, and this implies $\Phi_{c, r_{\mathrm{g}, \mathfrak{z}}}\left(b_{1}\right)(x) \sqsubseteq$ Ord $\Phi_{c, r_{\mathrm{g}, \mathfrak{z}}}\left(b_{2}\right)(x)$. Therefore Cond. $2 \mathrm{~b}$ is satisfied.

Lemma A.6. The algebra $r_{\mathrm{g}, \mathfrak{z}}: F_{\mathrm{g}} \operatorname{Ord}_{\leq \mathfrak{z}} \rightarrow \operatorname{Ord}_{\leq \mathfrak{z}}$ is corecursive.

Proof. Let $c: X \rightarrow F_{\mathrm{g}} X$ be an $F_{\mathrm{g}}$-coalgebra. It suffices to show that $\Phi_{c, r_{\mathrm{g}, \mathfrak{z}}}$ (Def. III.2) has a unique fixed point.

By Lem. A.5 the poset $\left(\operatorname{Ord}_{\leq \mathfrak{z}} X, \sqsubseteq\right.$ Ord $)$ (here $\varlimsup_{\text {Ord }}$ denotes the pointwise extension of $\sqsubseteq$ Ord over $\operatorname{Ord}_{\leq_{\mathfrak{z}}}$ ) is a complete lattice. Therefore we can construct a transfinite sequence

$$
\begin{aligned}
\perp_{\mathbf{O r d}_{\leq \mathfrak{z}}} \sqsubseteq \text { Ord } \Phi_{c, r_{\mathrm{g}, \mathfrak{z}}}( & \perp_{\left.\mathbf{O r d}_{\leq \mathfrak{z}}\right) \sqsubseteq \text { Ord }} \cdots \\
& \sqsubseteq \text { Ord } \Phi_{c, r_{\mathrm{g}, \mathfrak{z}}}^{\mathfrak{a}}\left(\perp_{\mathbf{O r d}_{\leq \mathfrak{z}}}\right) \sqsubseteq \text { Ord } \cdots
\end{aligned}
$$

as in Thm. I.18,2. By Thm. I.18,2, there exists an ordinal $\mathfrak{n}$ such that $\Phi_{c, r_{\mathrm{g}, \mathfrak{z}}}^{\mathfrak{n}}\left(\perp_{\mathbf{O r d}_{\leq \mathfrak{z}}}\right)$ is the least fixed point of $\Phi_{c, r_{\mathrm{g}, \mathfrak{z}}}$.

It remains to show that this is the unique fixed point. Let $f_{1}, f_{2}: X \rightarrow \operatorname{Ord}_{\leq \mathfrak{z}}$ be fixed points of $\Phi_{c, r_{\mathrm{g}, \mathfrak{z}}}$. We prove $f_{1}(x)=\mathfrak{a} \Leftrightarrow f_{2}(x)=\mathfrak{a}$ for each $x \in X$ and $\mathfrak{a}<\mathfrak{z}$ by transfinite induction on $\mathfrak{a}$.

For the base case, we have:

$$
\begin{aligned}
& f_{1}(x)=0 \\
& \Leftrightarrow r_{\mathrm{g}, \mathfrak{z}} \circ F_{\mathrm{g}} f_{1} \circ c(x)=0 \\
& \Leftrightarrow c_{2}(x)=1 \\
& \Leftrightarrow r_{\mathrm{g}, \mathfrak{z}} \circ F_{\mathrm{g}} f_{2} \circ c(x)=0 \\
& \Leftrightarrow f_{2}(x)=0
\end{aligned}
$$$$
\text { ( } \left.f_{1} \text { is a fixed point of } \Phi_{c, r_{\mathrm{g}, \mathfrak{z}}}\right)
$$$$
\text { (by def. of } r_{\mathrm{g}, \mathfrak{z}} \text { and } F_{\mathrm{g}} \text { ) }
$$$$
\text { (by def. of } F_{\mathrm{g}} \text { and } r_{\mathrm{g}, \mathfrak{z}} \text { ) }
$$$$
\text { ( } f_{2} \text { is a fixed point of } \Phi_{c, r_{\mathrm{g}, \mathfrak{z}}} \text { ). }
$$

Let $0<\mathfrak{a}<\mathfrak{z}$ and assume $f(x)=\mathfrak{a}^{\prime}$ iff $g(x)=\mathfrak{a}^{\prime}$ for each $x \in X$ and $\mathfrak{a}^{\prime}<\mathfrak{a}$. Note here that by $\mathfrak{a}<\mathfrak{z}$, we have $\mathfrak{a} \widehat{+} 1=\mathfrak{a}+1$. Hence we have:

$$
\begin{aligned}
& f_{1}(x)=\mathfrak{a} \\
& \Leftrightarrow r_{\mathrm{g}, \mathfrak{z}} \circ F_{\mathrm{g}} f_{1} \circ c(x)=\mathfrak{a} \quad\left(f_{1} \text { is a fixed point of } \Phi_{c, r_{\mathrm{g}, \mathfrak{z}}}\right) \\
& \Leftrightarrow c_{2}(x)=0 \text { and } \min _{A \in c_{1}(x)} \sup _{x^{\prime} \in A} f_{1}\left(x^{\prime}\right)=\mathfrak{a}-1 \\
& \left.\Leftrightarrow c_{2}(x)=0 \text { and } \min _{A \in c_{1}(x)} \sup _{x^{\prime} \in A} f_{2}\left(x^{\prime}\right)=\mathfrak{a}-1 \quad \text { (by def. of } r_{\mathrm{g}, \mathfrak{z}} \text { and } F_{\mathrm{g}}\right) \\
& \Leftrightarrow \Phi_{c, r_{\mathrm{g}, \mathfrak{z}}}\left(f_{2}\right)(x)=\mathfrak{a} \quad \text { (by IH) } \\
& \Leftrightarrow f_{2}(x)=\mathfrak{a} \quad\left(f_{2} \text { is a fixed point of } \Phi_{c, r_{\mathrm{g}, \mathfrak{z}}}\right)
\end{aligned}
$$

Hence we have $f_{1}(x)=\mathfrak{a}$ iff $f_{2}(x)=\mathfrak{a}$ for each $x \in X$ and $\mathfrak{a}<\mathfrak{z}$. This immediately implies that $f_{1}(x)=\mathfrak{z}$ iff $f_{2}(x)=\mathfrak{z}$ for each $x \in X$.

Proof (Prop. A.4). We prove that the conditions in Def. III.10 are satisfied.

Cond. 17. Let $(\Gamma, t) \in F_{\mathrm{g}} \operatorname{Ord}_{\leq \mathfrak{z}}=\mathcal{P}^{2} r_{\mathrm{g}, \mathfrak{z}} \times\{0,1\}$ and assume that $\sigma_{\mathrm{g}} \circ F_{\mathrm{g}} q_{\mathrm{g}, \mathfrak{z}}(\Gamma, t)=0$. Then by the definitions of $\sigma_{\mathrm{g}}, q_{\mathrm{g}, \mathfrak{z}}$ and $F_{\mathrm{g}}$, we have $t=0$ and $\forall A \in \Gamma . \mathfrak{z} \in A$. Hence by the definition of $r_{\mathfrak{g}, \mathfrak{z}}$, we have $r_{\mathfrak{g}, \mathfrak{z}}(\Gamma, t)=\mathfrak{z}$. Therefore by the definition of $q_{\mathrm{g}, \mathfrak{z}}$, we have $q_{\mathrm{g}, \mathfrak{z}} \circ r_{\mathrm{g}, \mathfrak{z}}(\Gamma, t)=0$. Hence Cond. 1 is satisfied.

Cond. 25. Already proved in Lem. A.5

Cond. 3. By the definition of $q_{\mathrm{g}, \mathfrak{z}}: R \rightarrow\{0,1\}$, for $a_{1}, a_{2} \in$ $\operatorname{Ord}_{\leq \mathfrak{z}}, a_{1} \leq a_{2}$ implies $q_{\mathrm{g}, \mathfrak{z}}\left(a_{1}\right) \geq q_{\mathrm{g}, \mathfrak{z}}\left(a_{2}\right)$ (here each $\leq$ denotes the usual order). Therefore $q_{\mathrm{g}, \mathfrak{z}}$ is monotone.

By its definition, $q_{\mathrm{g}, \mathfrak{z}}(\mathfrak{z})=0$ and hence $q_{\mathrm{g}, \mathfrak{z}}$ is strict.

For a subset $K \subseteq \operatorname{Ord}_{\leq_{\mathfrak{z}}}$, it is easy to see that $\bigsqcup_{a \in K}\left(q_{\mathrm{g}, \mathfrak{z}}(a)\right) \leq q_{\mathrm{g}, \mathfrak{z}}\left(\bigsqcup_{a \in K} a\right)$ is satisfied. We prove the opposite direction. Assume that $q_{\mathrm{g}, \mathfrak{z}}\left(\bigsqcup_{a \in K} a\right)=1$. Then by the definition of $q_{\mathrm{g}, \mathfrak{z}}, \bigsqcup_{a \in K} a=\mathfrak{n}$ for some $\mathfrak{n}<\mathfrak{z}$. By the definition of $\sqsubseteq$ Ord, this implies $a=\mathfrak{n}$ for some $a \in K$. Therefore we have $q_{\mathrm{g}, \mathfrak{z}}(a)=1$, and this implies $\bigsqcup_{a \in K}\left(q_{\mathrm{g}, \mathfrak{z}}(a)\right)=1$. Hence $q_{\mathrm{g}, \mathfrak{z}}\left(\bigsqcup_{a \in K} a\right) \leq \bigsqcup_{a \in K}\left(q_{\mathrm{g}, \mathfrak{z}}(a)\right)$ holds, and $q_{\mathrm{g}, \mathfrak{z}}$ is continuous.

Cond. 4. Already proved in Lem. A.6.

Hence $\left(r_{\mathrm{g}, \mathfrak{z}}, q_{\mathrm{g}, \mathfrak{z}}, \sqsubseteq\right.$ Ord $)$ is a ranking domain.

Proposition A.7. Let $\left(r_{\mathrm{g}, \mathfrak{z}}, q_{\mathrm{g}, \mathfrak{z}}\right.$, in Prop. A.4 For each function $b: X \rightarrow \operatorname{Ord}_{\leq \mathfrak{z}}$, we have the followings.

1) Let $c: X \rightarrow F_{\mathrm{g}} X$ be an $F_{\mathrm{g}}$-coalgebra. A function $b$ : $X \rightarrow \operatorname{Ord}_{\leq \mathfrak{z}}$ is a ranking arrow (Def. III.11) for c wrt. $\left(r_{\mathrm{g}, \mathfrak{z}}, q_{\mathrm{g}, \mathfrak{z}}, \sqsubseteq\right.$ Ord $)$ if and only if $b$ is a ranking function (Def. II.4) for $G^{c}$ and $\mathrm{Acc}^{c}$.

2) Let $G=\left(X_{\max }, x_{\min }, \tau\right)$ be a game structure and Acc $\subseteq X_{\max }$ be a set of accepting states. A function $b: X_{\max } \rightarrow \operatorname{Ord}_{\leq \mathfrak{z}}$ is a ranking function for $G$ and Acc if and only if $b$ is a ranking arrow for $c^{G, \text { Acc }}$ wrt. $\left(r_{\mathrm{g}, \mathfrak{z}}, q_{\mathrm{g}, \mathfrak{z}}, \sqsubseteq\right.$ Ord $)$.

3) $b(x)<\mathfrak{z}$ iff $q_{\mathrm{g}, \mathfrak{z}} \circ b(x)=1$.

Proof. 
1. For $x \in X$ such that $c(x)=(\Gamma, t)$ we write $c_{1}(x)$ and $c_{2}(x)$ for $\Gamma$ and $t$ respectively. We have:

$b: X \rightarrow$ Ord $_{\leq \mathfrak{z}}$ is a ranking arrow for $c$

$\Leftrightarrow \forall x \in X . b(x) \sqsubseteq$ Ord $r_{\mathrm{g}, \mathfrak{z}} \circ F_{\mathrm{g}} b \circ c(x)$

$\Leftrightarrow \forall x \in X \cdot r_{\mathrm{g}, \mathfrak{z}} \circ F_{\mathrm{g}} b \circ c(x) \leq b(x)$

(by Def. III.11)

(by the definition of $\sqsubseteq$ Ord)

$\Leftrightarrow \forall x \in X .\left(c_{2}(x)=0 \Rightarrow \min _{A \in c_{1}(x)} \sup _{x^{\prime} \in A} b\left(x^{\prime}\right) \widehat{+} 1 \leq b(x)\right)$

(by the definitions of $r_{\mathrm{g}, \mathfrak{z}}$ and $F_{\mathrm{g}}$ )

$\Leftrightarrow \forall x \in X_{\max }^{c}$

$\left(x \notin \mathrm{Acc}^{c} \Rightarrow \min _{y:(x, y) \in \tau^{c}} \sup _{x^{\prime}:\left(y, x^{\prime}\right) \in \tau^{c}} b\left(x^{\prime}\right) \widehat{+} 1 \leq b(x)\right)$

(by the definitions of $G^{c}$ and $\mathrm{Acc}^{c}$ )

$\Leftrightarrow b: X \rightarrow$ Ord $_{\leq \mathfrak{z}}$ is a ranking function for $G^{c}$ and $\mathrm{Acc}^{c}$

(by Def. I.4).

Hence Cond. 1 is satisfied.

2. This is proved in a similar manner to Cond. 1

3. Immediate from the definition of $q_{\mathrm{g}, \mathfrak{z}}$.

\section{B. Proof of Lem. III.13}

The basic idea of the proof is similar to the proof of Thm. I.18, 2 in [29].

Proof. Let $\mathfrak{m}$ be an ordinal such that $|L|<|\mathfrak{m}|$. As $\mid\left\{f^{\mathfrak{a}}(l) \in\right.$ $L \mid \mathfrak{a} \leq \mathfrak{m}\}|\leq| L \mid$, there exist ordinals $\mathfrak{a}, \mathfrak{a}^{\prime}$ such that $f^{\mathfrak{a}}(l)=$ $f^{\mathfrak{a}^{\prime}}(l)$. Without loss of generality, we assume $\mathfrak{a}<\mathfrak{a}^{\prime}$.

By monotonicity of $f$ and that $l \sqsubseteq f(l)$, we have

$$
f^{\mathfrak{a}}(l)=f^{\mathfrak{a}+1}(l)=\cdots=f^{\mathfrak{a}^{\prime}}(l) .
$$

This concludes the proof.

\section{Proof of Prop. III.18}

Proof. By $(\mid c)_{r}=r \circ F(|c|)_{r} \circ c$ and $q \circ r=\sigma \circ F q$, we have:

$$
q \circ(|c|)_{r}=\Phi_{c, \sigma}\left(q \circ(|c|)_{r}\right) .
$$

This means that $q \circ(|c|)_{r}$ is a fixed point of $\Phi_{c, \sigma}$. As $\llbracket \mu \sigma \rrbracket_{c}$ is the least fixed point of $\Phi_{c, \sigma}$ (Def. III.5), we have

$$
q \circ(c)_{r} \beth_{\Omega} \llbracket \mu \sigma \rrbracket_{c} .
$$

Together with Thm. II.12 we have $q \circ(\mid c)_{r}=\llbracket \mu \sigma \rrbracket_{c}$.

\section{Proof of Prop. IV.2}

Proof. Recall that Reach $_{\text {Acc }^{c}}$ is defined as the function $f$ : $X \rightarrow[0,1]$ in Def. II.9. We first show that this $f$ is a fixed point of $\Phi_{c, \sigma_{\mathrm{p}}}$. For $x \in X$, we have:

$$
\begin{aligned}
& \Phi_{c, \sigma_{\mathrm{p}}}(f)(x) \\
& =\left(\sigma_{\mathrm{p}} \circ F_{\mathrm{p}} f \circ c\right)(x) \\
& = \begin{cases}1 & \left(c_{2}(x)=1\right) \\
\sum_{x^{\prime} \in \operatorname{supp}\left(c_{1}(x)\right)} c_{1}(x)\left(x^{\prime}\right) \cdot f\left(x^{\prime}\right) & \left(c_{2}(x)=0\right)\end{cases}
\end{aligned}
$$$$
=\left\{\begin{array}{lr}
1 & \left.\begin{array}{rl}
\text { by def. of } & \left.\sigma_{\mathrm{p}} \text { and } F_{\mathrm{p}}\right) \\
\sum_{x^{\prime} \in \operatorname{supp}\left(c_{1}(x)\right)} c_{1}(x)\left(x^{\prime}\right) \cdot \lim _{n \rightarrow \infty} f_{n}\left(x^{\prime}\right) & \left(c_{2}(x)=0\right)
\end{array}\right)
\end{array}\right.
$$

(by def. of $f$ )

$$
\begin{aligned}
& = \begin{cases}1 & \left(c_{2}(x)=1\right) \\
\lim _{n \rightarrow \infty} \sum_{x^{\prime} \in \operatorname{supp}\left(c_{1}(x)\right)} c_{1}(x)\left(x^{\prime}\right) \cdot f_{n}\left(x^{\prime}\right) & \left(c_{2}(x)=0\right)\end{cases} \\
& =\left\{\begin{array}{ll}
1 & \left(c_{2}(x)=1\right) \\
\lim _{n \rightarrow \infty} f_{n+1}(x) & \left(c_{2}(x)=0\right)
\end{array} \quad \text { (by def. of } f_{n}\right) \\
& =\lim _{n \rightarrow \infty} f_{n}(x) \quad \text { (by def. of } f_{n} \text { ) } \\
& =f(x) \quad \text { (by def. of } f \text { ). }
\end{aligned}
$$

Hence $f$ is a fixed point of $\Phi_{c, \sigma_{\mathrm{p}}}$.

It remains to show that $f$ is the least fixed point. Let $f^{\prime}$ : $X \rightarrow[0,1]$ be a fixed point of $\Phi_{c, \sigma_{\mathrm{p}}}$.

We prove $f(x) \leq f^{\prime}(x)$ for each $x \in X$. To this end, by the definition of $f$, it suffices to prove $f_{n}(x) \leq f^{\prime}(x)$ for each $x \in X$ and $n \in \mathbb{N}$. We prove this by induction on $n$.

For $n=0$, it is immediate from that $f_{0}(x)=0$.

For $n>0$, we have:

$$
\begin{aligned}
& f_{n}(x) \\
& = \begin{cases}1 & \left(c_{2}(x)=1\right) \\
\sum_{x^{\prime} \in \operatorname{supp}\left(c_{1}(x)\right)} c_{1}(x)\left(x^{\prime}\right) \cdot f_{n-1}\left(x^{\prime}\right) & \left(c_{2}(x)=0\right)\end{cases} \\
& \text { (by def. of } f_{n} \text { ) } \\
& \leq\left\{\begin{array}{ll}
1 & \left(c_{2}(x)=1\right) \\
\sum_{x^{\prime} \in \operatorname{supp}\left(c_{1}(x)\right)} c_{1}(x)\left(x^{\prime}\right) \cdot f^{\prime}\left(x^{\prime}\right) & \left(c_{2}(x)=0\right)
\end{array}\right. \text { (by IH) } \\
& \left.=\left(\sigma_{\mathrm{p}} \circ F_{\mathrm{p}} f^{\prime} \circ c\right)(x) \quad \text { (by def. of } F_{\mathrm{p}} \text { and } \sigma_{\mathrm{p}}\right) \\
& =f^{\prime}(x) \\
& \text { ( } f^{\prime} \text { is a fixed point) } \text {. }
\end{aligned}
$$

Hence we have $f_{n}(x) \leq f^{\prime}(x)$ for each $x \in X$ and $n \in \mathbb{N}$, and this implies $f(x) \leq f^{\prime}(x)$ for each $x \in X$. Therefore $f$ is the least fixed point of $\Phi_{c, \sigma_{\mathrm{p}}}$.

\section{E. Proof of Prop. IV.3}

Proof. 
1. For each $b^{\prime}: X \rightarrow[0, \infty]$, we have:

$b^{\prime}$ is an $\varepsilon$-additive ranking supermartingale

$$
\begin{aligned}
\Leftrightarrow(\forall x & \in X . \\
c_{2}(x) & =0 \\
\Rightarrow & \left.\sum_{x^{\prime} \in \operatorname{supp}\left(c_{1}(x)\right)} c_{1}(x)\left(x^{\prime}\right) \cdot b^{\prime}\left(x^{\prime}\right)+\varepsilon \leq b^{\prime}(x)\right)
\end{aligned}
$$

(by Def. II.11)

$\Leftrightarrow \forall x \in X \cdot r_{\mathrm{p}, \varepsilon}^{\prime} \circ F_{\mathrm{p}} b^{\prime} \circ c(x) \leq b^{\prime}(x)$ (by def. of $r_{\mathrm{p}, \varepsilon}^{\prime}$ )

$\Leftrightarrow b^{\prime}$ is a (categorical) ranking arrow wrt. $\left(r_{\mathrm{p}, \varepsilon}^{\prime}, q_{\mathrm{p}}^{\prime}, \sqsubseteq_{[0, \infty]}\right)$

(by Def. II.11) .

Hence Cond. 11 holds.

2. Immediate from the definition of $q_{\mathrm{p}}^{\prime}$.

\section{F. Proof of Prop. IV.4}

\section{Proof.}

Cond. 마 Let $(\psi, t) \in F_{\mathrm{p}}[0, \infty]=\mathcal{D}[0, \infty] \times\{0,1\}$. Then we have:

$$
\begin{aligned}
& q_{\mathrm{p}}^{\prime} \circ r_{\mathrm{p}, \varepsilon}^{\prime}(\psi, t) \\
& = \begin{cases}q_{\mathrm{p}}^{\prime}(0) & (t=1) \\
q_{\mathrm{p}}^{\prime}\left(\sum_{a \in \operatorname{supp}(\psi)} a \cdot \psi(a)+\varepsilon\right) & (t=0)\end{cases} \\
& \text { (by def. of } r_{\mathrm{p}, \varepsilon}^{\prime} \text { ) } \\
& = \begin{cases}1 & \left(t=1 \text { or } \sum_{a \in \operatorname{supp}(\psi)} a \cdot \psi(a)+\varepsilon<\infty\right) \\
0 & \text { (otherwise) }\end{cases} \\
& \leq \begin{cases}1 & (t=1 \text { or } \psi([0, \infty))=1) \\
0 & \text { (otherwise })\end{cases} \\
& \leq \begin{cases}1 & (t=1) \\
\psi([0, \infty)) & (\text { otherwise })\end{cases} \\
& =\left\{\begin{array}{ll}
1 & (t=1) \\
1 \cdot \mathcal{D} q_{\mathrm{p}}(\psi)(1) & (\text { otherwise })
\end{array} \quad \text { (by def. of } q_{\mathrm{p}}^{\prime}\right) \\
& =\sigma_{\mathrm{p}} \circ F_{\mathrm{p}} q_{\mathrm{p}}^{\prime}(\psi, t) \quad \text { (by def. of } \sigma_{\mathrm{p}} \text { ). }
\end{aligned}
$$

Hence we have $q_{\mathrm{p}}^{\prime} \circ r_{\mathrm{p}, \varepsilon}^{\prime} \leq \sigma_{\mathrm{p}} \circ F_{\mathrm{p}} q_{\mathrm{p}}^{\prime}$.

Cond. 2. It is easy to see that Cond. 2a is satisfied.

We prove that Cond. $2 \mathrm{~b}$ is satisfied. Let $b_{1}, b_{2}: X \rightarrow[0, \infty]$ and assume that $b_{1} \sqsubseteq_{[0, \infty]} b_{2}$. For each $x \in X$ such that $c(x)=(\varphi, t) \in F_{\mathrm{p}} X=\mathcal{D} X \times\{0,1\}$, we have:

$$
\begin{aligned}
& \Phi_{c, r_{\mathrm{p}, \varepsilon}^{\prime}}\left(b_{1}\right)(x) \\
& =r_{\mathrm{p}, \varepsilon}^{\prime} \circ F_{\mathrm{p}} b_{1}(\varphi, t) \\
& =\left\{\begin{array}{lr}
1 & (t=1) \\
\sum_{x \in \operatorname{supp}(\varphi)} \varphi(x) \cdot b_{1}(x)+\varepsilon & (t=0)
\end{array}\right.
\end{aligned}
$$

(by def. of $r_{\mathrm{p}, \varepsilon}^{\prime}$ )

$$
\leq \begin{cases}1 & (t=1) \\ \sum_{x \in \operatorname{supp}(\varphi)} \varphi(x) \cdot b_{2}(x)+\varepsilon & (t=1)\end{cases}
$$

$$
\begin{array}{lr}
=r_{\mathrm{p}, \varepsilon}^{\prime} \circ F_{\mathrm{p}} b_{2}(\varphi, t) & \text { (by def. of } \left.r_{\mathrm{p}, \varepsilon}^{\prime}\right) \\
=\Phi_{c, r_{\mathrm{p}, \varepsilon}^{\prime}}\left(b_{2}\right)(x) & \text { (by def. of } \left.\Phi_{c, r_{\mathrm{p}, \varepsilon}^{\prime}}\right) .
\end{array}
$$

Therefore Cond. $2 \mathrm{~b}$ is satisfied.

Cond. 35: By the definition of $q_{\mathrm{p}}^{\prime}$, for each $a_{1}, a_{2} \in[0, \infty]$, $a_{1} \geq a_{2}$ implies $q_{\mathrm{p}}^{\prime}\left(a_{1}\right) \leq q_{\mathrm{p}}^{\prime}\left(a_{2}\right)$ (here each $\leq$ denotes the standard order). Hence $q_{\mathrm{p}}^{\prime}$ is monotone.

By the definition, we have $q_{\mathrm{p}}^{\prime}(\infty)=0$. Hence $q_{\mathrm{p}}^{\prime}$ is strict.

We prove that $q_{\mathrm{p}}^{\prime}$ is continuous. Namely, for each subset $K \subseteq[0, \infty]$, we prove

$$
\bigsqcup_{a \in K}\left(q_{\mathrm{p}}^{\prime}(a)\right)=q_{\mathrm{p}}^{\prime}\left(\bigsqcup_{a \in K} a\right) .
$$

It is easy to prove (LHS) $\leq$ (RHS). We prove the opposite direction. To this end, by the definition of $q_{\mathrm{p}}^{\prime}$, it suffices to prove that if the right-hand side is 1 then the left-hand side is also 1 . We can prove it as follows (here $\bigsqcup$ denotes the supremum with respect to $\sqsubseteq_{[0, \infty]}$, and $\bigwedge$ denotes the infimum with respect to the ordinary order over $[0,1])$.

$$
\begin{array}{rlr}
q_{\mathrm{p}}^{\prime}\left(\bigsqcup_{a \in K} a\right)=1 & \Rightarrow \bigsqcup_{a \in K} a<\infty & \text { (by def. of } q_{\mathrm{p}}^{\prime} \text { ) } \\
& \left.\Rightarrow \bigwedge_{a \in K} a<\infty \quad \text { (by def. of } \sqsubseteq_{[0, \infty]}\right) \\
& \Rightarrow \exists a \in K \cdot a<\infty & \\
& \Rightarrow \exists a \in K \cdot q_{\mathrm{p}}^{\prime}(a)=1 \quad \text { (by def. of } q_{\mathrm{p}}^{\prime} \text { ) } \\
& \Rightarrow \bigsqcup_{a \in K}\left(q_{\mathrm{p}}^{\prime}(a)\right)=1 &
\end{array}
$$

Hence $q_{\mathrm{p}}^{\prime}$ is continuous.

This concludes the proof.

\section{G. Multiplicative Ranking Supermartingale, Categorically}

Definition A.8. Let $M=(X, \tau)$ be a PTS. Let $\alpha \in(0,1)$. A function $b: X \rightarrow[0, \infty]$ is an $\alpha$-multiplicative ranking supermartingale if we have

$$
\sum_{x^{\prime} \in \operatorname{supp}(\tau(x))} \tau(x)\left(x^{\prime}\right) \cdot b\left(x^{\prime}\right) \leq \alpha \cdot b(x)
$$

for each $x \in X \backslash$ Acc, and moreover there exists $\delta>0$ such that $b(x) \geq \delta$ for each $x \in X \backslash$ Acc.

The following is an attempt to define a ranking domain for $\alpha$-multiplicative supermartingales. Let us fix real numbers $\alpha \in(0,1)$ and $\delta>0$, and define an $F_{\mathrm{p}}$-algebra $r_{\mathrm{pm}, \alpha}^{\prime}$ : $F_{\mathrm{p}}[\alpha \delta, \infty] \rightarrow[\alpha \delta, \infty]$, an arrow $q_{\mathrm{p}}^{\prime}:[0, \infty] \rightarrow[0,1]$ and a partial order $\sqsubseteq_{[\alpha \delta, \infty]}$ over $[\alpha \delta, \infty]$ as follows.

1) For each $(\psi, t) \in F_{\mathrm{p}}[\alpha \delta, \infty]=\mathcal{D}[\alpha \delta, \infty] \times\{0,1\}$,

$$
\begin{aligned}
& r_{\mathrm{pm}, \alpha}^{\prime}(\psi, t)= \\
& \begin{cases}\alpha \delta & (t=1) \\
\frac{1}{\alpha} \cdot\left(\sum_{a \in \operatorname{supp}(\psi)} a \cdot \psi(a)\right) & (\text { otherwise }) .\end{cases}
\end{aligned}
$$

2) $q_{\mathrm{p}}^{\prime}(\infty)=0$ and $q_{\mathrm{p}}^{\prime}(a)=1$ if $a<\infty$.

3) $a \sqsubseteq_{[\alpha \delta, \infty]} b \stackrel{\text { def. }}{\Leftrightarrow} a \geq b$ (note the direction).

The following proposition is analogous to Prop. IV.3. 
Proposition A.9. In this setting, for each $c: X \rightarrow F_{\mathrm{p}} X$, we have the following.

a) Let $b^{\prime}: X \rightarrow[\alpha \delta, \infty]$ and assume $b^{\prime} \sqsubseteq_{[\alpha \delta, \infty]} r_{\mathrm{pm}, \alpha}^{\prime} \circ$ $F_{\mathrm{p}} b^{\prime} \circ$ c. If we define $b: X \rightarrow[0, \infty]$ by $b(x)=b^{\prime}(x)$, then $b$ is an $\alpha$-multiplicative ranking supermartingale.

b) Let $b: X \rightarrow[0, \infty]$ be an $\alpha$-multiplicative ranking supermartingale such that $b(x) \geq \delta$ if $x \in X \backslash$ Acc. If we define $b^{\prime}: X \rightarrow[\alpha \delta, \infty]$ by $b^{\prime}(x)=\max \{\alpha \delta, b(x)\}$, then $b^{\prime}$ satisfies $b^{\prime} \sqsubseteq_{[\alpha \delta, \infty]} r_{\mathrm{pm}, \alpha}^{\prime} \circ F_{\mathrm{p}} b^{\prime} \circ c$.

c) For each $b^{\prime}: X \rightarrow[0, \infty]$, we have $b^{\prime}(x) \neq \infty$ iff $q_{\mathrm{p}}^{\prime} \circ b^{\prime}(x)=1$.

Proof.

a). Let $x \in X \backslash$ Acc. Then we have:

$$
\begin{aligned}
& \sum_{x^{\prime} \in \operatorname{supp}(\tau(x))} \tau(x)\left(x^{\prime}\right) \cdot b\left(x^{\prime}\right) \\
= & \sum_{x^{\prime} \in \operatorname{supp}\left(c_{1}(x)\right)} c_{1}(x)\left(x^{\prime}\right) \cdot b^{\prime}\left(x^{\prime}\right) \\
= & \alpha \cdot\left(r_{\mathrm{pm}, \alpha}^{\prime} \circ F_{\mathrm{p}} b^{\prime} \circ c\right)(x) \\
\leq & \alpha \cdot b^{\prime}(x) \\
= & \alpha \cdot b(x) .
\end{aligned}
$$

By the inequality above, we also have

$$
b(x) \geq \frac{1}{\alpha} \sum_{x^{\prime} \in \operatorname{supp}\left(c_{1}(x)\right)} c_{1}(x)\left(x^{\prime}\right) \cdot b^{\prime}\left(x^{\prime}\right) .
$$

As $b^{\prime}\left(x^{\prime}\right) \geq \alpha \delta$ holds for each $x^{\prime} \in X$, we have $b(x) \geq \delta$. Therefore $b$ is an $\alpha$-multiplicative ranking supermartingale. b). For each $x \in X$, we have:

$$
\begin{aligned}
& \left(r_{\mathrm{pm}, \alpha}^{\prime} \circ F_{\mathrm{p}} b^{\prime} \circ c\right)(x) \\
& = \begin{cases}\alpha \delta & \left(c_{2}(x)=1\right) \\
\frac{1}{\alpha} \sum_{x^{\prime} \in \operatorname{supp}\left(c_{1}(x)\right)} c_{1}(x)\left(x^{\prime}\right) \cdot b^{\prime}\left(x^{\prime}\right) & \left(c_{2}(x)=0\right)\end{cases} \\
& = \begin{cases}\alpha \delta & (x \in \mathrm{Acc}) \\
\frac{1}{\alpha} \sum_{x^{\prime} \in \operatorname{supp}(\tau(x))} \tau(x)\left(x^{\prime}\right) \cdot b\left(x^{\prime}\right) & (x \in X \backslash \mathrm{Acc})\end{cases} \\
& \leq \begin{cases}\alpha \delta & (x \in \mathrm{Acc}) \\
\frac{1}{\alpha} \cdot \alpha b(x) & (x \in X \backslash \mathrm{Acc})\end{cases} \\
& \leq b^{\prime}(x) .
\end{aligned}
$$

Therefore by the definition of $\sqsubseteq_{[\alpha \delta, \infty]}$, we have $b^{\prime} \sqsubseteq_{[\alpha \delta, \infty]}$ $r_{\mathrm{pm}, \alpha}^{\prime} \circ F_{\mathrm{p}} b^{\prime} \circ c$.

c). Immediate from the definition of $q_{\mathrm{p}}^{\prime}$.

Therefore the triple $\left(r_{\mathrm{pm}, \alpha}^{\prime}, q_{\mathrm{p}}^{\prime}, \sqsubseteq_{[\alpha \delta, \infty]}\right)$ is suited for accommodating $\alpha$-multiplicative supermartingales in our categorical framework. However it is not a ranking domain. The following proposition is analogous to Prop. IV.4

Proposition A.10. The triple $\left(r_{\mathrm{pm}, \alpha}^{\prime}, q_{\mathrm{p}}^{\prime}, \sqsubseteq_{[0, \infty]}\right)$ introduced above satisfies the conditions of a ranking domain (Def. III.10), except for Cond. 4

Proof.
Cond. [7. Let $(\psi, t) \in F_{\mathrm{p}}[\alpha \delta, \infty]=\mathcal{D}[\alpha \delta, \infty] \times\{0,1\}$. Then we have:

$$
\begin{aligned}
& q_{\mathrm{p}}^{\prime} \circ r_{\mathrm{pm}, \alpha}^{\prime}(\psi, t) \\
& = \begin{cases}q_{\mathrm{p}}^{\prime}(\alpha \delta) & (t=1) \\
q_{\mathrm{p}}^{\prime}\left(\frac{1}{\alpha} \cdot \sum_{a \in \operatorname{supp}(\psi)} a \cdot \psi(a)\right) & (t=0)\end{cases} \\
& \text { (by def. of } r_{\mathrm{pm}, \alpha}^{\prime} \text { ) } \\
& = \begin{cases}1 & \left(t=1 \text { or } \frac{1}{\alpha} \cdot \sum_{a \in \operatorname{supp}(\psi)} a \cdot \psi(a)<\infty\right) \\
0 & \text { (otherwise) }\end{cases} \\
& \leq \begin{cases}1 & (t=1 \text { or } \psi([0, \infty))=1) \\
0 & \text { (otherwise })\end{cases} \\
& \leq \begin{cases}1 & (t=1) \\
\psi([0, \infty)) & (\text { otherwise })\end{cases} \\
& =\left\{\begin{array}{ll}
1 & (t=1) \\
1 \cdot \mathcal{D} q_{\mathrm{p}}(\psi)(1) & (\text { otherwise })
\end{array} \quad \text { (by def. of } q_{\mathrm{p}}^{\prime}\right) \\
& \left.=\sigma_{\mathrm{p}} \circ F_{\mathrm{p}} q_{\mathrm{p}}^{\prime}(\psi, t) \quad \text { (by def. of } \sigma_{\mathrm{p}}\right) \text {. }
\end{aligned}
$$

Hence we have $q_{\mathrm{p}}^{\prime} \circ r_{\mathrm{pm}, \alpha}^{\prime} \leq \sigma_{\mathrm{p}} \circ F_{\mathrm{p}} q_{\mathrm{p}}^{\prime}$.

Cond. 2. It is easy to see that Cond. 2a is satisfied.

We prove that Cond. $2 \mathrm{~b}$ is satisfied. Let $b_{1}, b_{2}: X \rightarrow$ $[\alpha \delta, \infty]$ and assume that $b_{1} \sqsubseteq_{[\alpha \delta, \infty]} b_{2}$. For each $x \in X$ such that $c(x)=(\varphi, t) \in F_{\mathrm{p}} X=\mathcal{D} X \times\{0,1\}$, we have:

$$
\begin{aligned}
& \Phi_{c, r_{\mathrm{pm}, \alpha}^{\prime}}\left(b_{1}\right)(x) \\
& \left.=r_{\mathrm{pm}, \alpha}^{\prime} \circ F_{\mathrm{p}} b_{1}(\varphi, t) \quad \text { (by def. of } \Phi_{c, r_{\mathrm{pm}, \alpha}^{\prime}}\right) \\
& = \begin{cases}1 & (t=1) \\
\frac{1}{\alpha} \cdot \sum_{x \in \operatorname{supp}(\varphi)} \varphi(x) \cdot b_{1}(x) & (t=0)\end{cases} \\
& \sqsubseteq_{[\alpha \delta, \infty]} \begin{cases}1 & (t=1) \\
\frac{1}{\alpha} \cdot \sum_{x \in \operatorname{supp}(\varphi)} \varphi(x) \cdot b_{2}(x) & (t=0)\end{cases} \\
& =r_{\mathrm{pm}, \alpha}^{\prime} \circ F_{\mathrm{p}} b_{2}(\varphi, t) \quad \text { (by def. of } r_{\mathrm{pm}, \alpha}^{\prime} \text { ) } \\
& =\Phi_{c, r_{\mathrm{pm}, \alpha}^{\prime}}\left(b_{2}\right)(x) \quad \text { (by def. of } \Phi_{c, r_{\mathrm{pm}, \alpha}^{\prime}} \text { ). }
\end{aligned}
$$

Therefore Cond. $2 \mathrm{~b}$ is satisfied.

Cond. 3. It is proved in a similar manner to the proof of Prop. IV.4

Example A.11. We define a coalgebra $c: X \rightarrow F_{\mathrm{p}} X$ as in Example IV.5. We fix $\alpha \in(0,1)$ and $\delta>0$. We assume that $\alpha>1 / 2$. For this coalgebra, we define arrows $b_{1}, b_{2}$ : $X \rightarrow[\alpha \delta, \infty]$ by: $b_{1}\left(x_{0}\right)=\frac{1}{2} \frac{1}{\alpha}\left(\frac{\alpha \delta}{2 \alpha-1}+\delta\right), b_{1}\left(x_{1}\right)=\frac{\alpha \delta}{2 \alpha-1}$, $b_{1}\left(x_{2}\right)=\delta$ and $b_{1}\left(x_{3}\right)=\alpha \delta$; and $b_{2}\left(x_{0}\right)=\infty, b_{2}\left(x_{1}\right)=$ $\infty, b_{2}\left(x_{2}\right)=\delta$ and $b_{2}\left(x_{3}\right)=\alpha \delta$. Both of these qualify as coalgebra-algebra homomorphisms from $c$ to $r_{\mathrm{pm}, \alpha}^{\prime}$. Therefore $r_{\mathrm{pm}, \alpha}^{\prime}$ is not corecursive. 


\section{H. Proof of Prop. IV.7}

We prove Prop. IV.7 in a similar manner to the proof of Prop. A.4 we first prove that $r_{\mathrm{p}}$ is a corecursive algebra separately. To this end, we first prove some lemmas.

Lemma A.12. The order $\sqsubseteq_{\mathcal{D N}}$ in Prop. IV.7 is a partial order and $\mathcal{D} \mathbb{N}_{\infty}$ is a complete lattice with respect to this order.

Sublemma A.13. For every nondecreasing function $G: \mathbb{N} \rightarrow$ $[0,1]$, there exists a unique distribution $\varphi$ over $\mathbb{N}_{\infty}$ such that $\varphi([0, a])=G(a)$ for each $a \in \mathbb{N}$.

Proof. We define a distribution $\varphi$ over $\mathbb{N}_{\infty}$ by

$$
\varphi(a)= \begin{cases}G(a) & (a=0) \\ G(a)-G(a-1) & (0<a<\infty) \\ 1-\lim _{a^{\prime} \rightarrow \infty} G\left(a^{\prime}\right) & (a=\infty) .\end{cases}
$$

As $G$ is nondecreasing, $\varphi(a) \geq 0$ for each $a$. By its definition, we have $\sum_{a \in \mathbb{N}_{\infty}} \varphi(a)=1$. Hence $\varphi$ is a distribution.

Let $\varphi^{\prime}$ be a distribution such that $\varphi^{\prime}([0, a])=G(a)$. Then we have $\varphi(0)=G(0)=\varphi^{\prime}([0,0])=\varphi^{\prime}(0)$. Moreover for each $a \in \mathbb{N} \backslash\{0\}$, we have:

$\varphi(a)=G(a)-G(a-1)=\varphi^{\prime}([0, a])-\varphi^{\prime}([0, a-1])=\varphi^{\prime}(a)$.

Therefore we have $\varphi(a)=\varphi^{\prime}(a)$ for each $a \in \mathbb{N}$, and this implies $\varphi(\infty)=\varphi^{\prime}(\infty)$. Hence uniqueness is proved, and this concludes the proof.

Proof (Lem. A.12). We first prove that $\sqsubseteq_{\mathcal{D} \mathbb{N}_{\infty}}$ is a partial order. Reflexivity and transitivity are immediate from those of the standard order $\leq$ over $[0,1]$. Assume that $\varphi \sqsubseteq_{\mathcal{D N}} \varphi^{\prime}$ and $\varphi^{\prime} \sqsubseteq \mathcal{D N}_{\infty} \varphi$. By the definition of $\subseteq_{\mathcal{D N}}$, we have $\varphi([0, a])=$ $\varphi^{\prime}([0, a])$ for each $a \in[0, \infty]$. Then by Sublem. A.13, we have $\varphi=\varphi^{\prime}$. Hence antisymmetry is also satisfied.

We prove that each subset $K \subseteq \mathcal{D} \mathbb{N}_{\infty}$ has the least upper bound. We define $G: \mathbb{N} \rightarrow[0,1]$ by $G(a)=\sup _{\varphi \in K} \varphi([0, a])$. Note that for each $\varphi \in K, a \leq b$ implies $\varphi([0, a]) \leq \varphi([0, b])$. Hence by the monotonicity of supremums, $G$ is nondecreasing. Therefore by Sublem. A.13, there exists a unique distribution $\varphi^{K} \in \mathcal{D N}_{\infty}$ such that $\varphi^{K}([0, a])=G(a)$ for each $a \in[0, \infty]$.

We prove that $\varphi^{K}$ is the least upper bound of $K$.

Let $\varphi \in K$. For each $a \in \mathbb{N}$, we have:

$$
\varphi([0, a]) \leq \sup _{\varphi^{\prime} \in K} \varphi^{\prime}([0, a])=\varphi^{K}([0, a]) .
$$

Hence by the definition of $\sqsubseteq_{\mathcal{D}}, \varphi$ is an upper bound of $K$.

Let $\varphi^{\prime}$ be an upper bound of $K$. Then by the definition of $\sqsubseteq_{\mathcal{D N}}$, we have $\varphi([0, a]) \leq \varphi^{\prime}([0, a])$ for each $\varphi \in K$ and $a \in \mathbb{N}$. Therefore we have $\varphi^{K}([0, a]) \leq \varphi^{\prime}([0, a])$, and this means $\varphi^{K} \sqsubseteq \mathcal{D N}_{\infty} \varphi^{\prime}$ by the definition of $\sqsubseteq \mathcal{D N}$. Hence $\varphi$ is the least upper bound of $K$.

In a similar manner, we can prove that each $K \subseteq \mathcal{D} \mathbb{N}_{\infty}$ has the greatest lower bound.

Lemma A.14. The algebra $r_{\mathrm{p}}: F_{\mathrm{p}} \mathcal{D} \mathbb{N}_{\infty} \rightarrow \mathcal{D} \mathbb{N}_{\infty}$ in Prop IV.7 satisfies Cond. 2 in Def. III.10

Proof. It is already proved in Lem. A.12 that Cond. 2a is satisfied.
We prove that Cond. $2 \mathrm{~b}$ is satisfied. Let $f_{1}, f_{2}: X \rightarrow \mathcal{D} \mathbb{N}_{\infty}$ and assume $f_{1} \sqsubseteq \mathcal{D N} \mathbb{N}_{\infty} f_{2}$. Let $x \in X$ and assume that $c(x)=$ $(\varphi, t) \in F_{\mathrm{p}} X$.

If $t=1$ then we have:

$$
r_{\mathrm{p}} \circ F_{\mathrm{p}} f_{1}(f)(\varphi, t)=r_{\mathrm{p}} \circ F_{\mathrm{p}} f_{2}(f)(\varphi, t)=\delta_{0} .
$$

Therefore we have $\Phi_{c, \sigma_{\mathrm{p}}}\left(f_{1}\right)(x)=\Phi_{c, \sigma_{\mathrm{p}}}\left(f_{2}\right)(x)$.

Let $t=0$ and $a \in \mathbb{N}$. If $a=0$, by the definition of $r_{\mathrm{p}}$, we have:

$$
r_{\mathrm{p}} \circ F_{\mathrm{p}} f_{1}(f)(\varphi, t)([0, a])=r_{\mathrm{p}} \circ F_{\mathrm{p}} f_{2}(f)(\varphi, t)([0, a])=0 .
$$

If $a>0$, we have:

$$
\begin{array}{lr}
r_{\mathrm{p}} \circ F_{\mathrm{p}} f_{1}(f)(\varphi, t)([0, a]) & \\
=\sum_{x \in \operatorname{supp}(\varphi)}\left(\varphi(x) \cdot f_{1}(x)([0, a-1])\right) & \left.\quad \text { (by def. of } r_{\mathrm{p}}\right) \\
\leq & \sum_{x \in \operatorname{supp}(\varphi)}\left(\varphi(x) \cdot f_{2}(x)([0, a-1])\right) \quad\left(\text { by } f_{1} \sqsubseteq \mathcal{D} \mathbb{N}_{\infty} f_{2}\right) \\
= & \left.\quad \text { (by def. of } r_{\mathrm{p}}\right) .
\end{array}
$$

Therefore by the definition of $\sqsubseteq \mathcal{D N}$, we have $r_{\mathrm{p}} \circ$ $F_{\mathrm{p}} f_{1}(\varphi, t) \sqsubseteq \mathcal{D} \mathbb{N}_{\infty} r_{\mathrm{p}} \circ F_{\mathrm{p}} f_{2}(\varphi, t)$. This means that $\Phi_{c, r_{\mathrm{p}}}$ is monotone. This concludes the proof.

Lemma A.15. The $F_{\mathrm{p}}$-algebra $r_{\mathrm{p}}: F_{\mathrm{p}} \mathcal{D} \mathbb{N}_{\infty} \rightarrow \mathcal{D N}_{\infty}$ in Prop. IV.7 is corecursive.

Proof. Let $c: X \rightarrow F_{\mathrm{p}} X$ be an $F_{\mathrm{p}}$-coalgebra. It suffices to show that the function $\Phi_{c, r_{\mathrm{p}}}:\left(\mathcal{D N}_{\infty}\right)^{X} \rightarrow\left(\mathcal{D N} \mathbb{N}_{\infty}\right)^{X}$ (Def. III.2) has a unique fixed point.

By Lem. A.14, the poset $\left(\left(\mathcal{D N} \mathbb{N}_{\infty}\right)^{X}, \sqsubseteq \mathcal{D N}_{\infty}\right)$ (here $\complement_{\mathcal{D N}}$ denotes the pointwise extension) is a complete lattice. Therefore we can construct a transfinite sequence

$$
\begin{aligned}
\perp_{\mathcal{D N}} \sqsubseteq \mathbb{D N}_{\infty} \Phi_{c, r_{\mathrm{p}}}\left(\perp_{\mathcal{D N} \mathbb{N}_{\infty}}\right) \sqsubseteq \mathcal{D} \mathbb{N}_{\infty} \cdots \\
\\
\sqsubseteq_{\mathcal{D} \mathbb{N}_{\infty}} \Phi_{c, r_{\mathrm{p}}}^{\mathfrak{a}}\left(\perp_{\mathcal{D} \mathbb{N}_{\infty}}\right) \sqsubseteq \mathcal{D N}_{\infty} \cdots
\end{aligned}
$$

as in Thm. ПI.182. By Thm. 【I.18,2, there exists an ordinal $\mathfrak{n}$ such that $\Phi_{c, r_{\mathrm{p}}}^{\mathfrak{n}}\left(\perp_{\mathcal{D N}}\right)$ is the least fixed point of $\Phi_{c, r_{\mathrm{p}}}$.

It remains to show that this is a unique fixed point. Let $f_{1}, f_{2}: X \rightarrow \mathcal{D N}_{\infty}$ be fixed points of $\Phi_{c, r_{\mathrm{p}}}$. We prove

$$
f_{1}(x)(a)=f_{2}(x)(a)
$$

for each $x \in X$ and $a \in \mathbb{N}$. For each $x \in X$ such that $(\varphi, t)=c(x)$, we write $c_{1}(x)$ and $c_{2}(x)$ for $\varphi \in \mathcal{D} X$ and $t \in\{0,1\}$ respectively.

We first prove (10) for each $x \in X$ such that $c_{2}(x)=1$. For such $x$, we have:

$$
\begin{array}{rlr}
f_{1}(x)(a) & =\Phi_{c, r_{\mathrm{p}}}\left(f_{1}\right)(x) & \left(f_{1} \text { is a fixed point }\right) \\
& =r_{\mathrm{p}} \circ F_{\mathrm{p}} f_{1} \circ c(x) & \\
& =\delta_{0} \\
& =r_{\mathrm{p}} \circ F_{\mathrm{p}} f_{2} \circ c(x) \\
& =\Phi_{c, r_{\mathrm{p}}}\left(f_{2}\right)(x) \quad\left(f_{2} \text { is a fixed point }\right) \\
& =g(x) .
\end{array}
$$


Hence we have $f_{1}(x)(a)=f_{2}(x)(a)$ for each $a$.

It remains to prove (10) for each $x \in X$ such that $c_{2}(x)=0$.

We prove it by the induction on $a$.

If $a=0$ then by the definition of $r_{\mathrm{p}}$, we have $f_{1}(x)(a)=$ $f_{2}(x)(a)=0$.

Let $a>0$ and assume $f_{1}\left(x^{\prime}\right)\left(a^{\prime}\right)=f_{2}\left(x^{\prime}\right)\left(a^{\prime}\right)$ for each $x^{\prime} \in X$ and $a^{\prime}<a$. Then we have:

$$
\begin{aligned}
& f_{1}(x)(a) \\
& =r_{\mathrm{p}} \circ F_{\mathrm{p}} f_{1} \circ c(x)(a) \\
& \text { ( } f_{1} \text { is a fixed point) } \\
& =\sum_{x^{\prime} \in \operatorname{supp}\left(c_{1}(x)\right)} c_{1}(x)\left(x^{\prime}\right) \cdot f_{1}\left(x^{\prime}\right)(a-1) \quad\left(\text { by def. of } r_{\mathrm{p}}\right) \\
& =\sum_{x^{\prime} \in \operatorname{supp}\left(c_{1}(x)\right)} c_{1}(x)\left(x^{\prime}\right) \cdot f_{2}\left(x^{\prime}\right)(a-1) \\
& =r_{\mathrm{p}} \circ F_{\mathrm{p}} f \circ c(x)(a) \\
& =f_{2}(x)(a)
\end{aligned}
$$

Therefore we have $f_{1}(x)(a)=f_{2}(x)(a)$ for each $x \in X$ and $a \in \mathbb{N}$. Note that this implies that $f_{x}(x)(\infty)=f_{2}(x)(\infty)$ for each $x \in X$. This concludes the proof.

Proof (Prop. IV.7). We prove that $\left(r_{\mathrm{p}}, q_{\mathrm{p}}, \sqsubseteq \mathcal{D N}_{\infty}\right)$ satisfies the conditions in Def. III.10.

Cond. 17. Let $(\Gamma, t) \in F_{\mathrm{p}} \mathcal{D} \mathbb{N}_{\infty}=\mathcal{D}^{2} \mathbb{N}_{\infty} \times\{0,1\}$. If $t=1$ then by the definitions of $r_{\mathrm{p}}$ and $\sigma_{\mathrm{p}}$, we have:

$q_{\mathrm{p}} \circ r_{\mathrm{p}}(\Gamma, t)=q_{\mathrm{p}}\left(\delta_{0}\right)=1=\sigma_{\mathrm{p}}\left(\mathcal{D} q_{\mathrm{p}}(\Gamma), t\right)=\sigma_{\mathrm{p}} \circ F_{\mathrm{p}} q_{\mathrm{p}}(\Gamma, t)$.

Assume $t=0$. Then we have:

$$
\begin{aligned}
& q_{\mathrm{p}} \circ r_{\mathrm{p}}(\Gamma, t) \\
& \left.=r_{\mathrm{p}}(\Gamma, 1)([0, \infty)) \quad \text { (by def. of } q_{\mathrm{p}}\right) \\
& =\sum_{a=0}^{\infty} r_{\mathrm{p}}(\Gamma, 1)(a) \\
& =\sum_{a=1}^{\infty} \sum_{\gamma \in \operatorname{supp}(\Gamma)} \Gamma(\gamma) \cdot \gamma(a-1) \quad \text { (by def. of } r_{\mathrm{p}} \text { ) } \\
& =\sum_{a=0}^{\infty} \sum_{\gamma \in \operatorname{supp}(\Gamma)} \Gamma(\gamma) \cdot \gamma(a) \\
& =\sum_{\gamma \in \operatorname{supp}(\Gamma)} \Gamma(\gamma) \cdot\left(\sum_{a=0}^{\infty} \gamma(a)\right) \\
& =\sum_{\gamma \in \operatorname{supp}(\Gamma)} \Gamma(\gamma) \cdot q_{\mathrm{p}}(\gamma) \\
& =\sigma_{\mathrm{p}} \circ F_{\mathrm{p}} q_{\mathrm{p}}(\Gamma, t) \\
& \text { (by def. of } q_{\mathrm{p}} \text { ) } \\
& \text { (by def. of } \sigma_{\mathrm{p}} \text { ). }
\end{aligned}
$$

Hence Cond. 1 is satisfied. Note also that we have $q_{\mathrm{p}} \circ r_{\mathrm{p}}=$ $\sigma_{\mathrm{p}} \circ F_{\mathrm{p}} q_{\mathrm{p}}$.

Cond. 2. We have already shown in Lem. A.14

Cond. 3. We first prove that $q_{\mathrm{p}}$ is monotone. Let $f_{1}, f_{2}$ : $X \rightarrow \mathcal{D} \mathbb{N}_{\infty}$ and assume that $f \sqsubseteq \mathcal{D N} \infty$. Then we have:

$$
\begin{array}{rlrl}
q_{\mathrm{p}} \circ f_{1}(x) & =f_{1}(x)([0, \infty)) & & \text { (by def. of } \left.q_{\mathrm{p}}\right) \\
& =\lim _{a \rightarrow \infty} f_{1}(x)([0, a]) & \\
& \leq \lim _{a \rightarrow \infty} f_{2}(x)([0, a]) \quad\left(\text { by } f_{1} \sqsubseteq \mathcal{D N} \mathbb{N}_{\infty} f_{2}\right)
\end{array}
$$

$$
\begin{aligned}
& =f_{2}(x)([0, \infty)) \\
& \left.=q_{\mathrm{p}} \circ f_{2}(x) \quad \text { (by def. of } q_{\mathrm{p}}\right) .
\end{aligned}
$$

Hence we have $q_{\mathrm{p}} \circ f_{1} \leq q_{\mathrm{p}} \circ f_{2}$, and therefore Cond. 3 was proved.

By the definition of $q_{\mathrm{p}}$, we have $q_{\mathrm{p}}\left(\delta_{\infty}\right)=0$. Therefore $q_{\mathrm{p}}$ is strict.

We prove that $q_{\mathrm{p}}$ is continuous. By the definition of $q_{\mathrm{p}}$, it suffices to prove $\left(\bigsqcup_{\gamma \in K} \gamma\right)([0, \infty))=\sup _{\gamma \in K}(\gamma([0, \infty)))$. Note that the supremum on the left-hand side is taken with respect to $\sqsubseteq \mathcal{D} \mathbb{N}_{\infty}$ over $\mathcal{D} \mathbb{N}_{\infty}$ while the latter is taken with respect to the ordinary order over $[0,1]$. We have:

$$
\begin{aligned}
& \left(\bigsqcup_{\gamma \in K} \gamma\right)([0, \infty)) \\
& =\lim _{a \rightarrow \infty}\left(\bigsqcup_{\gamma \in K} \gamma\right)([0, a]) \\
& =\lim _{a \rightarrow \infty} \sup _{\gamma \in K}(\gamma([0, a])) \\
& =\sup _{\gamma \in K} \lim _{a \rightarrow \infty}(\gamma([0, a])) \\
& =\sup _{\gamma \in K}(\gamma([0, \infty))) .
\end{aligned}
$$$$
=\lim _{a \rightarrow \infty} \sup _{\gamma \in K}(\gamma([0, a])) \quad \text { (see the proof of Lem. A.12) }
$$

Therefore $q_{\mathrm{p}}$ is continuous.

Cond. 4. We have already shown in Lem. A.15

Hence the triple $\left(r_{\mathrm{p}}, q_{\mathrm{p}}, \sqsubseteq \mathcal{D} \mathbb{N}_{\infty}\right)$ is a ranking domain.

\section{Soundness of Additive Ranking Supermartingale, Cate- gorically}

In $₫$ IV-A we have seen that the triple $\left(r_{\mathrm{p}, \varepsilon}^{\prime}, q_{\mathrm{p}}^{\prime}, \sqsubseteq_{[0, \infty]}\right)$, which captures the definition of $\varepsilon$-additive ranking supermartingales, is not a ranking domain because $r_{\mathrm{p}, \varepsilon}^{\prime}$ is not a corecursive algebra. Hence we cannot prove soundness of $\varepsilon$ additive ranking supermartingales directly using our categorical framework. In this section, we show that its soundness is proved via that of distribution-valued ranking functions. More concretely, we have the following proposition.

Proposition A.16. We define triples $\left(r_{\mathrm{p}}, q_{\mathrm{p}}, \sqsubseteq \mathcal{D} \mathbb{N}_{\infty}\right)$ and $\left(r_{\mathrm{p}, \varepsilon}^{\prime}, q_{\mathrm{p}}^{\prime}, \sqsubseteq_{[0, \infty]}\right)$ as in Prop. IV.7 and Prop. IV.3 respectively. Let $c: X \rightarrow F_{\mathrm{p}} X$ be an $F_{\mathrm{p}}$-coalgebra. Then we have:

$$
\begin{aligned}
\exists b^{\prime}: X \rightarrow[0, \infty] . b^{\prime} \sqsubseteq_{[0, \infty]} \Phi_{c, r_{\mathrm{p}, \varepsilon}^{\prime}}\left(b^{\prime}\right) \\
\Rightarrow \exists b: X \rightarrow \mathcal{D} \mathbb{N}_{\infty} . \\
b \sqsubseteq \mathcal{D} \mathbb{N}_{\infty} \Phi_{c, r_{\mathrm{p}}}(b), \text { and } \\
\forall x \in X .\left(q_{\mathrm{p}}^{\prime} \circ b^{\prime}(x)=1 \Rightarrow q_{\mathrm{p}} \circ b(x)=1\right) .
\end{aligned}
$$

This proposition is an immediate corollary of the following lemma.

Lemma A.17. We assume the conditions in Prop. A.16 We define a function $p_{\mathrm{p}}: \mathcal{D} \mathbb{N}_{\infty} \rightarrow[0, \infty]$ by

$$
p_{\mathrm{p}}(\gamma)=\varepsilon \cdot \sum_{a \in \operatorname{supp}(\gamma)} a \cdot \gamma(a) .
$$

Then the following statements hold. 
1) $q_{\mathrm{p}}^{\prime} \circ p_{\mathrm{p}} \leq q_{\mathrm{p}}$ (here $\leq$ denotes the pointwise extension over $\left.\mathcal{D} \mathbb{N}_{\infty}([0, \infty])\right)$.

2) Let $b^{\prime}: X \rightarrow[0, \infty]$ and assume $b^{\prime} \sqsubseteq_{[0, \infty]} \Phi_{c, r_{\mathrm{p}, \varepsilon}^{\prime}}\left(b^{\prime}\right)$. As $r_{\mathrm{p}}: F_{\mathrm{p}} \mathcal{D} \mathbb{N}_{\infty} \rightarrow \mathcal{D} \mathbb{N}_{\infty}$ is corecursive (Prop. IV.7), there exists a unique function $(c)_{r_{\mathrm{p}}}: X \rightarrow \mathcal{D} \mathbb{N}_{\infty}$ such that $(c \mid)_{r_{\mathrm{p}}}=\Phi_{c, r_{\mathrm{p}}}\left((c \mid)_{r_{\mathrm{p}}}\right)$. For this function, we have $b^{\prime} \sqsubseteq[0, \infty] p_{\mathrm{p}} \circ(c) r_{\mathrm{p}}$.

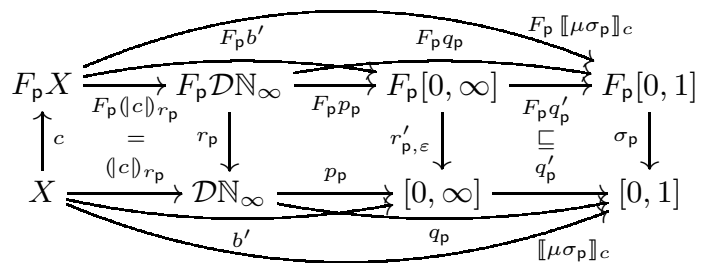

Sublemma A.18. For the function $p_{\mathrm{p}}: \mathcal{D N}_{\infty} \rightarrow[0, \infty]$ in Lem. A.17 we have the following.

$$
p_{\mathrm{p}} \circ r_{\mathrm{p}}=r_{\mathrm{p}, \varepsilon}^{\prime} \circ F_{\mathrm{p}} p_{\mathrm{p}}
$$

Proof. Let $(\Gamma, t) \in F_{\mathrm{p}} \mathcal{D} \mathbb{N}_{\infty}=\mathcal{D}^{2} \mathbb{N}_{\infty} \times\{0,1\}$. If $t=1$, then by the definitions of $r_{\mathrm{p}}$ and $r_{\mathrm{p}, \varepsilon}^{\prime}$, we have

$$
p_{\mathrm{p}} \circ r_{\mathrm{p}}(\Gamma, t)=r_{\mathrm{p}, \varepsilon}^{\prime} \circ F_{\mathrm{p}} p_{\mathrm{p}}(\Gamma, t)=0 .
$$

Let $t=0$. Then we have:

$$
\begin{aligned}
& p_{\mathrm{p}} \circ r_{\mathrm{p}}(\Gamma, t) \\
& =\varepsilon \cdot \sum_{a \in \mathbb{N}_{\infty} \backslash\{0\}} a \cdot r_{\mathrm{p}}(\Gamma, t)(a) \quad \text { (by def. of } p_{\mathrm{p}} \text { ) } \\
& \left.=\varepsilon \cdot \sum_{a \in \mathbb{N}_{\infty} \backslash\{0\}} a \cdot \sum_{\gamma \in \operatorname{supp}(\Gamma)} \Gamma(\gamma) \cdot \gamma(a-1) \quad \text { (by def. of } r_{\mathbf{p}}\right) \\
& =\varepsilon \cdot \sum_{a \in \mathbb{N}_{\infty}}(a+1) \cdot \sum_{\gamma \in \operatorname{supp}(\Gamma)} \Gamma(\gamma) \cdot \gamma(a) \\
& =\varepsilon \cdot \sum_{a \in \mathbb{N}_{\infty}} a \cdot \sum_{\gamma \in \operatorname{supp}(\Gamma)} \Gamma(\gamma) \cdot \gamma(a) \\
& +\varepsilon \cdot \sum_{a \in \mathbb{N}_{\infty}} \sum_{\gamma \in \operatorname{supp}(\Gamma)} \Gamma(\gamma) \cdot \gamma(a) \\
& =\sum_{a \in \mathbb{N}_{\infty}}\left(a \cdot \varepsilon \cdot \sum_{\gamma \in \operatorname{supp}(\Gamma)} \Gamma(\gamma) \cdot \gamma(a)\right)+\varepsilon \\
& =\sum_{\gamma \in \operatorname{supp}(\Gamma)}\left(\Gamma(\gamma) \cdot \varepsilon \cdot \sum_{a \in \mathbb{N}_{\infty}} a \cdot \gamma(a)\right)+\varepsilon \\
& =\sum_{\gamma \in \operatorname{supp}(\Gamma)}\left(\Gamma(\gamma) \cdot p_{\mathrm{p}}(\gamma)\right)+\varepsilon \\
& =\sum_{b \in \operatorname{supp}\left(\mathcal{D} p_{\mathrm{p}}(\Gamma)\right)}\left(b \cdot \sum_{\gamma^{\prime} \in p_{\mathrm{p}}^{-1}(b)} \Gamma\left(\gamma^{\prime}\right)\right)+\varepsilon \\
& =\sum_{b \in \operatorname{supp}\left(\mathcal{D} p_{\mathrm{p}}(\Gamma)\right)}\left(b \cdot \mathcal{D} p_{\mathrm{p}}(\Gamma)(b)\right)+\varepsilon \\
& =r_{\mathrm{p}, \varepsilon}^{\prime} \circ F_{\mathrm{p}} p_{\mathrm{p}}(\Gamma, t) \\
& \text { (by def. of } r_{p, \varepsilon}^{\prime} \text { ). }
\end{aligned}
$$

This concludes the proof.

Sublemma A.19. The $F_{\mathrm{p}}$-algebra $r_{\mathrm{p}, \varepsilon}^{\prime}: F_{\mathrm{p}}[0, \infty] \rightarrow[0, \infty]$ in Prop. IV.3 satisfies the following conditions.

1) The poset $([0, \infty], \sqsubseteq[0, \infty])$ is a complete lattice.
2) For each $F_{\mathrm{p}}$-coalgebra $c: X \rightarrow F_{\mathrm{p}} X$, the function $\Phi_{c, r_{\mathrm{p}, \varepsilon}^{\prime}}:[0, \infty]^{X} \rightarrow[0, \infty]^{X}$ in Def. III.5 is monotone with respect to the pointwise extension of $\sqsubseteq[0, \infty]$.

Moreover the triple $\left(r_{\mathrm{p}}: F_{\mathrm{p}} \mathcal{D} \mathbb{N}_{\infty} \rightarrow \mathcal{D} \mathbb{N}_{\infty}, p_{\mathrm{p}}: \mathcal{D} \mathbb{N}_{\infty} \rightarrow\right.$ $[0, \infty], \sqsubseteq_{\mathcal{D N}}$ ) (see Prop. IV.7 and Lem. A.17) satisfies the following conditions.

3) We have $p_{\mathrm{p}} \circ r_{\mathrm{p}} \sqsupseteq_{[0, \infty]} r_{\mathrm{p}, \varepsilon}^{\prime} \circ F_{\mathrm{p}} p_{\mathrm{p}}$ between arrows $F_{\mathrm{p}} \mathcal{D} \mathbb{N}_{\infty} \rightarrow[0, \infty]$.

4) The following conditions are satisfied by $r_{\mathrm{p}}$ :

a) the poset $\left(\mathcal{D} \mathbb{N}_{\infty}, \sqsubseteq \mathcal{D} \mathbb{N}_{\infty}\right)$ has the greatest element $\top_{\mathcal{D N}}$;

b) the poset $\left(\mathcal{D} \mathbb{N}_{\infty}, \sqsubseteq \mathcal{D} \mathbb{N}_{\infty}\right)$ is $\omega^{\mathrm{op}}$-complete (i.e. for each decreasing sequence $\varphi_{0} \sqsupseteq_{\mathcal{D N}} \varphi_{1} \sqsupseteq_{\mathcal{D} \mathbb{N}_{\infty}}$ $\cdots$ in $\mathcal{D} \mathbb{N}_{\infty}$, the infimum $\prod_{i \in \omega} \varphi_{i}$ exists);

c) for each $c: X \rightarrow F_{\mathrm{p}} X$, the function $\Phi_{c, r_{\mathrm{p}}}:$ $\left.(\mathcal{D N})^{X} \rightarrow(\mathcal{D N})_{\infty}\right)^{X}$ (Def. III.2) is monotone and moreover $\omega^{\mathrm{op}}$-continuous, i.e. for each decreasing sequence $f_{0} \sqsupseteq_{\mathcal{D N}} \quad f_{1} \sqsupseteq_{\mathcal{D N}} \cdots$ in $\left(\mathcal{D} \mathbb{N}_{\infty}\right)^{X}$ wrt. the pointwise extension of $\sqsubseteq \mathcal{D} \mathbb{N}_{\infty}$, we have $\Phi_{c, r_{\mathrm{p}}}\left(\prod_{i \in \omega} f_{i}\right)=\prod_{i \in \omega} \Phi_{c, r_{\mathrm{p}}}\left(f_{i}\right)$.

5) The function $p_{\mathrm{p}}: \mathcal{D} \mathbb{N}_{\infty} \rightarrow[0, \infty]$ is monotone (i.e. $a \sqsubseteq \mathcal{D} \mathbb{N}_{\infty} b \Rightarrow p_{\mathrm{p}}(a) \sqsubseteq_{[0, \infty]} p_{\mathrm{p}}(b)$ ), top-preserving (i.e. $\left.p_{\mathrm{p}}\left(\top_{\mathcal{D N}}\right)=\top_{[0, \infty]}\right)$ and $\omega^{\mathrm{op}}$-continuous (i.e. each decreasing sequence $\varphi_{0} \sqsupseteq_{\mathcal{D N}} \varphi_{1} \sqsupseteq_{\mathcal{D N}} \cdots$ in $\mathcal{D} \mathbb{N}_{\infty}$, we have $\left.p_{\mathrm{p}}\left(\prod_{i \in \omega} \varphi_{i}\right)=\prod_{i \in \omega} p_{\mathrm{p}}\left(\varphi_{i}\right)\right)$. Note that in the last equality, the infimum in the left-hand side is take wrt. $\sqsubseteq \mathcal{D N}$ while the one in the right-hand side is taken wrt. $\sqsubseteq[0, \infty] \cdot$

6) The algebra $r_{\mathrm{p}}: F_{\mathrm{p}} \mathcal{D} \mathbb{N}_{\infty} \rightarrow \mathcal{D} \mathbb{N}_{\infty}$ is corecursive.

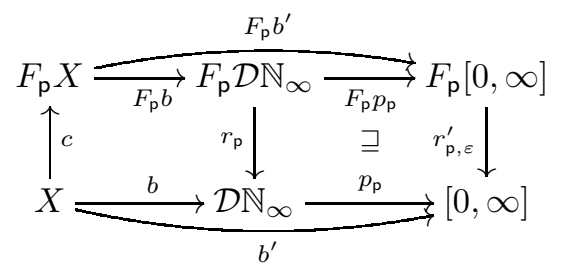

Proof.

1. Easy.

2. Already proved in Prop. IV.4

3. Immediate from Sublem. A.18

4. It is easy to see that the Dirac distribution $\delta_{0}$ concentrated at 0 is the greatest element in $\left(\mathcal{D N} \mathbb{N}_{\infty}, \sqsubseteq \mathcal{D} \mathbb{N}_{\infty}\right)$. Hence Cond. 4a is satisfied. Cond. 4b is immediate from that $\left.\left((\mathcal{D N})_{\infty}\right)^{X}, \sqsubseteq \mathcal{D N} \mathbb{N}_{\infty}\right)$ is a complete lattice (Lem. A.12).

We prove that Cond. $4 \mathrm{c}$ is satisfied. Monotonicity of $\Phi_{c, r_{\mathrm{p}}}$ is already proved in Prop. IV.7. We prove that $\Phi_{c, r_{\mathrm{p}}}$ is $\omega^{\text {op }}$ continuous. To this end, it suffices to prove the following equality for each $x \in X$.

$$
\Phi_{c, r_{\mathrm{p}}}\left(\prod_{i \in \omega} f_{i}\right)(x)=\prod_{i \in \omega}\left(\Phi_{c, r_{\mathrm{p}}}\left(f_{i}\right)(x)\right)
$$

Note that $\prod$ on the right-hand side denotes the infimum with respect to $\sqsubseteq \mathcal{D N} \mathbb{N}_{\infty}$ while that on the left-hand side denotes the infimum with respect to its pointwise extension. Let $(\delta, t)=$ $c(x)$. 
If $t=1$, then by the definition of $r_{\mathrm{p}}$, we have $\Phi_{c, r_{\mathrm{p}}}(f)(x)=$ $\delta_{0}$ for each $f: X \rightarrow \mathcal{D} \mathbb{N}_{\infty}$. Hence we have (12).

If $t=0$, for each $a \in \mathbb{N}$, we have:

$$
\begin{aligned}
& \left(\prod_{i \in \omega}\left(\Phi_{c, r_{\mathrm{p}}}\left(f_{i}\right)(x)\right)\right)([0, a]) \\
& =\inf _{i \in \omega}\left(\Phi_{c, r_{\mathrm{p}}}\left(f_{i}\right)(x)([0, a])\right) \quad(\text { see the proof of Lem. A.12] }) \\
& \left.=\inf _{i \in \omega} \sum_{x \in \operatorname{supp}(\delta)} \delta(x) \cdot\left(f_{i}(x)([0, a-1])\right) \quad \text { (by def. of } r_{\mathrm{p}}\right) \\
& =\sum_{x \in \operatorname{supp}(\delta)} \delta(x) \cdot\left(\inf _{i \in \omega} f_{i}(x)([0, a-1])\right)
\end{aligned}
$$

(by the dominated convergence theorem)

$=\sum_{x \in \operatorname{supp}(\delta)} \delta(x) \cdot\left(\prod_{i \in \omega} f_{i}\right)(x)([0, a-1])$

(see the proof of Lem. A.12)

$$
=\Phi_{c, r_{\mathrm{p}}}\left(\prod_{i \in \omega} f_{i}\right)(x)([0, a])
$$

(by def. of $\Phi_{c, r_{\mathrm{p}}}$ ).

By Sublem. A.13, this implies (12).

5. We first prove that $p_{\mathrm{p}}: \mathcal{D} \mathbb{N}_{\infty} \rightarrow[0, \infty]$ is monotone. Let $\varphi_{1}, \varphi_{2} \in \mathcal{D} \mathbb{N}_{\infty}$ and assume that $\varphi_{1} \sqsubseteq \mathcal{D N} \varphi_{\infty}$.

If $\varphi_{2}(\infty)=C>0$, then we have $\varphi_{2}([0, a]) \leq 1-C$ for each $a \in \mathbb{N}$. By the definition of $\sqsubseteq \mathcal{D} \mathbb{N}_{\infty}$, this implies $\varphi_{1}([0, a]) \leq 1-C$ for each $a$, and therefore we have $\varphi_{1}(\infty)>$ 0 . Hence in this case, we have $p_{\mathrm{p}}\left(\varphi_{1}\right)=p_{\mathrm{p}}\left(\varphi_{2}\right)=\infty$.

Let $\varphi_{2}(\infty)=0$. Then we have:

$$
\begin{aligned}
& p_{\mathrm{p}}\left(\varphi_{2}\right) \\
& \left.=\varepsilon \cdot \sum_{a=1}^{\infty} a \cdot \varphi_{2}(a) \quad \text { (by def. of } p_{\mathrm{p}}\right) \\
& =\lim _{n \rightarrow \infty} \varepsilon \cdot \sum_{a=1}^{n}\left(a \cdot \varphi_{2}([0, a])-a \cdot \varphi_{2}([0, a-1])\right) \\
& =\lim _{n \rightarrow \infty} \varepsilon \cdot\left(n \cdot \varphi_{2}([0, n])-\sum_{a=1}^{n} 1 \cdot \varphi_{2}([0, a-1])\right) \\
& =\lim _{n \rightarrow \infty} \varepsilon \cdot \sum_{a=1}^{n} \varphi_{2}([a, n]) \\
& =\varepsilon \cdot \sum_{a=1}^{\infty} \varphi_{2}([a, \infty]) \\
& =\varepsilon \cdot \sum_{a=1}^{\infty}\left(1-\varphi_{2}([0, a-1])\right) \\
& \left.\leq \varepsilon \cdot \sum_{a=1}^{\infty}\left(1-\varphi_{1}([0, a-1])\right) \quad \text { (by } \varphi_{1} \sqsubseteq \mathcal{D N} \varphi_{\infty}\right) \\
& =p_{\mathrm{p}}\left(\varphi_{1}\right)
\end{aligned}
$$

Hence we have $p_{\mathrm{p}}\left(\varphi_{1}\right) \sqsubseteq_{[0, \infty]} p_{\mathrm{p}}\left(\varphi_{2}\right)$ and therefore $p_{\mathrm{p}}$ is monotone.

By the definition of $p_{\mathrm{p}}, p_{\mathrm{p}}\left(\delta_{0}\right)=0$. Hence $p_{\mathrm{p}}$ is toppreserving.

We prove that $p_{\mathrm{p}}$ is $\omega^{\mathrm{op}}$-continuous. Let $\varphi_{0} \sqsupseteq_{\mathcal{D N}}$ $\varphi_{1} \sqsupseteq_{\mathcal{D N}} \cdots$ be a decreasing sequence in $\mathcal{D} \mathbb{N}_{\infty}$.
Assume $\left(\prod_{i \in \omega}\left(\varphi_{i}\right)\right)(\infty)=C>0$. Then for all $b \in \mathbb{N}$, there exists $i_{b} \in \omega$ such that $1-\varphi_{i}([0, b]) \geq \frac{C}{2}$ (c.f. the proof of Lem. A.12). Therefore for each $b \in \mathbb{N}$, we have:

$$
\begin{aligned}
\prod_{i \in \omega} p_{\mathrm{p}}\left(\varphi_{i}\right) & \geq p_{\mathrm{p}}\left(\varphi_{i_{b}}\right) \\
& \left.=\varepsilon \cdot \sum_{a=0}^{\infty} a \cdot \varphi_{i}(a) \quad \text { (by def. of } p_{\mathrm{p}}\right) \\
& \geq \varepsilon \cdot b \cdot(1-\varphi([0, b])) \\
& \left.\geq \varepsilon \cdot b \cdot\left(1-\frac{C}{2}\right)\right) .
\end{aligned}
$$

Hence we have

$$
p_{\mathrm{p}}\left(\prod_{i \in \omega}\left(\varphi_{i}\right)\right)=\infty=\prod_{i \in \omega} p_{\mathrm{p}}\left(\varphi_{i}\right)
$$

Assume $\left(\prod_{i \in \omega}\left(\varphi_{i}\right)\right)(\infty)=0$. Then we have:

$$
\begin{aligned}
& p_{\mathrm{p}}\left(\prod_{i \in \omega}\left(\varphi_{i}\right)\right) \\
& \left.=\varepsilon \cdot \sum_{a=0}^{\infty} a \cdot\left(\prod_{i \in \omega} \varphi_{i}\right)(a) \quad \text { (by def. of } p_{\mathrm{p}}\right) \\
& =\varepsilon \cdot \sum_{a=1}^{\infty}\left(1-\left(\prod_{i \in \omega} \varphi_{i}\right)([0, a-1])\right)
\end{aligned}
$$

(as in the proof of monotonicity of $p_{\mathrm{p}}$ )

$$
=\varepsilon \cdot \sum_{a=1}^{\infty}\left(1-\inf _{i \in \omega}\left(\varphi_{i}([0, a-1])\right)\right)
$$

(see the proof of Lem. A.12)

$$
\begin{array}{lr}
=\sup _{i \in \omega} \varepsilon \cdot \sum_{a=1}^{\infty}\left(1-\left(\varphi_{i}([0, a-1])\right)\right) & \\
=\sup _{i \in \omega} p_{\mathrm{p}}\left(\varphi_{i}\right) & \text { (as in the above) } \\
=\prod_{i \in \omega} p_{\mathrm{p}}\left(\varphi_{i}\right) & \text { (by def. of } \left.\sqsubseteq_{[0, \infty]}\right)
\end{array}
$$

Hence we have $\omega^{\mathrm{op}}$-continuity.

6. It is already proved in Lem. A.15

Proof (Lem. A.17).

1. For each $\varphi \in \mathcal{D} \mathbb{N}_{\infty}$, we have:

$$
\begin{aligned}
& q_{\mathrm{p}}^{\prime} \circ p_{\mathrm{p}}(\varphi) \\
& =q_{\mathrm{p}}^{\prime}\left(\sum_{a \in \mathbb{N}} a \cdot \varphi(a)\right) \\
& =\left\{\begin{array}{ll}
1 & \left(\sum_{a \in \mathbb{N}} a \cdot \varphi(a)<\infty\right) \\
0 & \text { (otherwise) }
\end{array} \quad \text { (by def. of } q_{\mathrm{p}}^{\prime}\right) \\
& \leq \varphi([0, \infty)) \\
& =q_{\mathrm{p}}(\varphi) \\
& \text { (by def. of } q_{p} \text { ). }
\end{aligned}
$$

Hence Cond. 1 is satisfied. 
2.

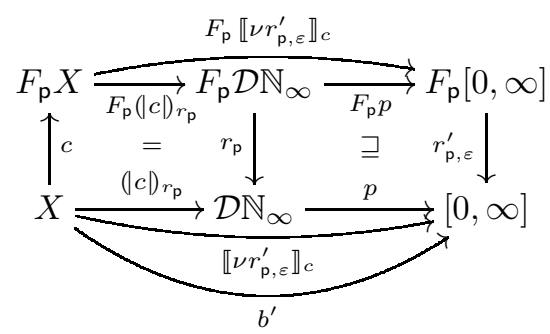

By Sublem. A.19112 the $F_{\mathrm{p}}$-modality $r_{\mathrm{p}, \varepsilon}^{\prime}: F_{\mathrm{p}}[0, \infty] \rightarrow$ $[0, \infty]$ satisfy dual conditions of Asm. II.7 Hence using the dual statement of Prop. III.8, we can show that $\Phi_{c, r_{p, \varepsilon}^{\prime}}$ : $[0, \infty]^{X} \rightarrow[0, \infty]^{X}$ has the greatest fixed point $\llbracket \nu r_{\mathrm{p}, \varepsilon}^{\prime} \rrbracket_{c}$ : $X \rightarrow[0, \infty]$ with respect to the pointwise extension of $\sqsubseteq_{[0, \infty]}$. By the Knaster-Tarski theorem, we have

$$
b^{\prime} \sqsubseteq_{[0, \infty]} \llbracket \nu r_{\mathrm{p}, \varepsilon}^{\prime} \rrbracket_{c} .
$$

By Sublem. A.1936 the triple $\left(r_{\mathrm{p}}: F_{\mathrm{p}} \mathcal{D} \mathbb{N}_{\infty} \rightarrow\right.$ $\left.\mathcal{D} \mathbb{N}_{\infty}, p_{\mathrm{p}}: \mathcal{D N}_{\infty} \rightarrow[0, \infty], \sqsubseteq_{[0, \infty]}\right)$ satisfies the dual conditions of the axioms of a ranking domain (Def. [II.10), except that the length of a transfinite sequence in $\left(\mathcal{D N} \mathbb{N}_{\infty}\right)^{X}$ is restricted to $\omega$. Note here that $(\mid c)_{r_{\mathrm{p}}}: X \rightarrow \mathcal{D} \mathbb{N}_{\infty}$ satisfies $\left(|c| r_{\mathrm{p}} \sqsupseteq_{\mathcal{D N}} \Phi_{c, r_{\mathrm{p}}}\left(|c| r_{r_{\mathrm{p}}}\right)\right.$ by its definition. Therefore in a similar manner to the proof of Thm. III.12, we can prove

$$
\left.\llbracket \nu r_{\mathrm{p}, \varepsilon}^{\prime} \rrbracket_{c} \sqsubseteq_{[0, \infty]} p_{\mathrm{p}} \circ(c)\right)_{r_{\mathrm{p}}} .
$$

By (14) and (13), we have $b^{\prime} \sqsubseteq_{[0, \infty]} p_{\mathrm{p}} \circ(\mid c)_{r}$.

Proof (Prop. A.16). Immediate from Lem. A.17.

We can now prove the soundness of additive ranking supermartingale (Thm. II.5) using our categorical framework as follows.

$\exists b^{\prime} . b^{\prime}$ is an $\varepsilon$-additive ranking supermartingale and $b^{\prime}(x) \neq \infty$ $\stackrel{\text { Prop. IV.3 }}{\Leftrightarrow} \exists b^{\prime} . b^{\prime} \sqsubseteq_{[0, \infty]} \Phi_{c, r_{\mathrm{p}, \varepsilon}^{\prime}}\left(b^{\prime}\right)$ and $q_{\mathrm{p}}^{\prime} \circ b^{\prime}(x)=1$

$\stackrel{\text { Prop. A.16 }}{\Rightarrow} \exists b . b$ is a ranking arrow wrt. $\left(r_{\mathrm{p}}, q_{\mathrm{p}}, \sqsubseteq \mathcal{D N}_{\infty}\right)$, and

$$
q_{\mathrm{p}} \circ b(x)=1
$$

$$
\begin{aligned}
& \stackrel{\text { Thm. III.12 }}{\Rightarrow} \llbracket \mu \sigma_{\mathrm{p}} \rrbracket_{c^{G, \text { Acc }}}(x)=1 \\
& \stackrel{\text { Prop. IV.2 }}{\Leftrightarrow} \operatorname{Reach}_{M, \text { Acc }}(x)=1
\end{aligned}
$$

\section{J. Soundness of Multiplicative Ranking Supermartingale, Categorically}

Proposition A.20. We define triples $\left(r_{\mathrm{p}}, q_{\mathrm{p}}, \sqsubseteq_{\mathcal{D N}}\right)$ and $\left(r_{\mathrm{pm}, \alpha}^{\prime}, q_{\mathrm{p}}^{\prime}, \sqsubseteq_{[\alpha \delta, \infty]}\right)$ as in Prop. IV.7 and Prop. A.9 respectively. Let $c: X \rightarrow F_{\mathrm{p}} X$ be an $F_{\mathrm{p}}$-coalgebra. Then we have:

$$
\begin{aligned}
\exists b^{\prime \prime}: X \rightarrow[\alpha \delta, \infty] . b^{\prime \prime} \sqsubseteq_{[\alpha \delta, \infty]} \Phi_{c, r_{\mathrm{pm}, \alpha}^{\prime}}\left(b^{\prime \prime}\right) \\
\Rightarrow \exists b: X \rightarrow \mathcal{D} \mathbb{N}_{\infty} . \\
\quad b \sqsubseteq \mathcal{D N}_{\infty} \Phi_{c, r_{\mathrm{p}}}(b), \text { and } \\
\quad \forall x \in X .\left(q_{\mathrm{p}}^{\prime} \circ b^{\prime}(x)=1 \Rightarrow q_{\mathrm{p}} \circ b(x)=1\right) .
\end{aligned}
$$

This proposition is an immediate corollary of Prop. A.16 and the following lemma.
Lemma A.21. We define triples $\left(r_{\mathrm{p}, \varepsilon}^{\prime}, q_{\mathrm{p}}^{\prime}, \sqsubseteq_{[0, \infty]}\right)$ and $\left(r_{\mathrm{pm}, \alpha}^{\prime}, q_{\mathrm{p}}^{\prime}, \sqsubseteq_{[0, \infty]}\right)$ as in Prop. IV.3 and Prop. A.9 respectively. We define a function $p_{\mathrm{p}}^{\prime}:[\alpha \delta, \infty] \rightarrow[0, \infty]$ as follows.

$$
p_{\mathrm{p}}^{\prime}(a)=\varepsilon \cdot\left(\left(\log _{\frac{1}{\alpha}} \frac{a}{\delta}\right)+1\right)
$$

Let $c: X \rightarrow F_{\mathrm{p}} X$ be an $F_{\mathrm{p}}$-coalgebra. Then we have:

$$
\begin{aligned}
& \exists b^{\prime \prime}: X \rightarrow[\alpha \delta, \infty] \cdot b^{\prime \prime} \sqsubseteq_{[\alpha \delta, \infty]} \Phi_{c, r_{\mathrm{pm}, \alpha}^{\prime}}\left(b^{\prime \prime}\right) \\
& \quad \Rightarrow p_{\mathrm{p}}^{\prime} \circ b^{\prime \prime} \sqsubseteq_{[0, \infty]} \Phi_{c, r_{\mathrm{p}, \varepsilon}^{\prime}}\left(p_{\mathrm{p}}^{\prime} \circ b^{\prime \prime}\right)
\end{aligned}
$$

Sublemma A.22. For the function $F[\alpha \delta, \infty] \stackrel{F p_{p}^{\prime}}{\longrightarrow} F[0, \infty]$ $p_{\mathrm{p}}^{\prime}:[\alpha \delta, \infty] \rightarrow[0, \infty]$ in Lem. A.21 $r_{\mathrm{pm}, \alpha}^{\prime} \downarrow \subseteq r_{\mathrm{p}, \varepsilon \downarrow}^{\prime} \downarrow$ we have $p_{\mathrm{p}}^{\prime} \circ r_{\mathrm{pm}, \alpha}^{\prime} \sqsubseteq[0, \infty] r_{\mathrm{p}, \varepsilon}^{\prime} \circ F_{\mathrm{p}} p_{\mathrm{p}}^{\prime} . \quad[\alpha \delta, \infty] \underset{p_{\mathrm{p}}^{\prime}}{\longrightarrow}[0, \infty]$

Proof. Let $(\psi, t) \in F_{\mathrm{p}}[\alpha \delta, \infty]=\mathcal{D}[\alpha \delta, \infty] \times\{0,1\}$. By the definition of $\sqsubseteq_{[0, \infty]}$, it suffices to prove $p_{\mathrm{p}}^{\prime} \circ r_{\mathrm{pm}, \alpha}^{\prime}(\psi, t) \geq$ $r_{\mathrm{p}, \varepsilon}^{\prime} \circ F_{\mathrm{p}} p_{\mathrm{p}}^{\prime}(\psi, t)$.

If $t=1$, then we have:

$$
\begin{array}{lr}
p_{\mathrm{p}}^{\prime} \circ r_{\mathrm{pm}, \alpha}^{\prime}(\psi, t) & \\
=p_{\mathrm{p}}^{\prime}(\alpha \delta) & \text { (by def. of } \left.r_{\mathrm{pm}, \alpha}^{\prime}\right) \\
=0 & \text { (by def. of } p_{\mathrm{p}}^{\prime} \text { ) } \\
=r_{\mathrm{p}, \varepsilon}^{\prime}\left(\mathcal{D} p_{\mathrm{p}}^{\prime}(\psi), t\right) & \text { (by def. of } \left.r_{\mathrm{p}, \varepsilon}^{\prime}\right) \\
=r_{\mathrm{p}, \varepsilon}^{\prime} \circ F_{\mathrm{p}} p_{\mathrm{p}}^{\prime}(\psi, t) . &
\end{array}
$$

Let $t=0$. Note that $\sum_{a \in \operatorname{supp}(\psi)} \psi(a)=1$. Hence we have: $p_{\mathrm{p}}^{\prime} \circ r_{\mathrm{pm}, \alpha}^{\prime}(\psi, t)$

$$
\begin{aligned}
& \left.=p_{\mathrm{p}}^{\prime}\left(\frac{1}{\alpha} \cdot\left(\sum_{a \in \operatorname{supp}(\psi)} a \cdot \psi(a)\right)\right) \quad \text { (by def. of } r_{\mathrm{pm}, \alpha}^{\prime}\right) \\
& =\varepsilon \cdot\left(\log _{\frac{1}{\alpha}} \frac{1}{\delta}\left(\frac{1}{\alpha} \cdot\left(\sum_{a \in \operatorname{supp}(\psi)} a \cdot \psi(a)\right)\right)+1\right)
\end{aligned}
$$

$$
\begin{aligned}
& =\varepsilon \cdot\left(\left(\log _{\frac{1}{\alpha}}\left(\sum_{a \in \operatorname{supp}(\psi)} \frac{a}{\delta} \cdot \psi(a)\right)\right)+2\right) \\
& \geq \varepsilon \cdot\left(\left(\sum_{a \in \operatorname{supp}(\psi)}\left(\log _{\frac{1}{\alpha}} \frac{a}{\delta}\right) \cdot \psi(a)\right)+2\right)
\end{aligned}
$$

$\left(\log _{\frac{1}{\alpha}}(-)\right.$ is an upward convex function)

$$
\begin{aligned}
& =\left(\sum_{a \in \operatorname{supp}(\psi)}\left(\varepsilon \cdot\left(\left(\log _{\frac{1}{\alpha}} \frac{a}{\delta}\right)+1\right)\right) \cdot \psi(a)\right)+\varepsilon \\
& \left.=r_{\mathrm{p}, \varepsilon}^{\prime} \circ F_{\mathrm{p}} p_{\mathrm{p}}^{\prime}(\varphi, t) \quad \text { (by def. of } p_{\mathrm{p}}^{\prime} \text { and } r_{\mathrm{p}, \varepsilon}^{\prime}\right) .
\end{aligned}
$$

This concludes the proof.

Proof (Lem. A.21).

$$
\begin{aligned}
& F X \stackrel{F b^{\prime \prime}}{\longrightarrow} F[\alpha \delta, \infty] \stackrel{F p_{\mathfrak{p}}^{\prime}}{\longrightarrow} F[0, \infty]
\end{aligned}
$$

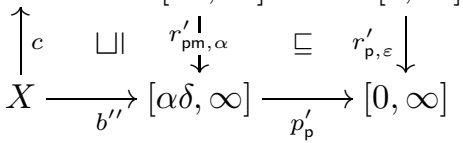


Let $b^{\prime \prime}: X \rightarrow[\alpha \delta, \infty]$ and assume that $b^{\prime \prime} \sqsubseteq_{[\alpha \delta, \infty]}$ $\Phi_{c, r_{\mathrm{pm}, \alpha}^{\prime}}\left(b^{\prime \prime}\right)$. Then we have:

$$
\begin{array}{lr}
p_{\mathrm{p}}^{\prime} \circ b^{\prime \prime} & \\
\sqsubseteq_{[0, \infty]} p_{\mathrm{p}}^{\prime} \circ r_{\mathrm{pm}, \alpha}^{\prime} \circ F_{\mathrm{p}} b^{\prime \prime} \circ c & \text { (by the assumption) } \\
\sqsubseteq_{[0, \infty] r_{\mathrm{p}, \varepsilon}^{\prime} \circ F_{\mathrm{p}} p_{\mathrm{p}}^{\prime} \circ F_{\mathrm{p}} b^{\prime \prime} \circ c} & \text { (by Sublem. A.22) } \\
=\Phi_{c, r_{\mathrm{p}, \varepsilon}^{\prime}}\left(p_{\mathrm{p}}^{\prime} \circ b^{\prime \prime}\right) & \text { (by definition). }
\end{array}
$$

This concludes the proof.

Proof (Prop. A.20). Immediate from Lem. A.21 and Prop. A.16

We can now prove the soundness of multiplicative ranking supermartingale using our categorical framework as follows.

$\exists b^{\prime \prime} . b^{\prime \prime}$ is an $\alpha$-multiplicative ranking supermartingale, and

$$
b^{\prime \prime}(x) \neq \infty
$$

$\stackrel{\text { Prop. A.9 }}{\Leftrightarrow} \exists b^{\prime} . b^{\prime} \sqsubseteq_{[0, \infty]} \Phi_{c, r_{\mathrm{p}, \varepsilon}^{\prime}}\left(b^{\prime}\right)$ and $q_{\mathrm{p}}^{\prime} \circ b^{\prime}(x)=1$

$\stackrel{\text { Prop.A.16 }}{\Rightarrow} \exists b . b$ is a ranking arrow wrt. $\left(r_{\mathrm{p}}, q_{\mathrm{p}}, \sqsubseteq \mathcal{D} \mathbb{N}_{\infty}\right)$, and

$$
q_{\mathrm{p}} \circ b(x)=1
$$

$$
\begin{aligned}
& \stackrel{\text { Thm. III.12 }}{\Rightarrow} \llbracket \mu \sigma_{\mathrm{p}} \rrbracket_{c^{G, \text { Acc }}}(x)=1 \\
& \stackrel{\text { Prop. IV.2 }}{\Leftrightarrow} \operatorname{Reach}_{M, \operatorname{Acc}}(x)=1
\end{aligned}
$$

\section{K. Proof of Prop. IV.12}

We prove that $r_{\mathrm{nc}, \gamma}$ is a corecursive algebra separately.

Lemma A.23. The algebra $r_{\mathrm{nc}, \gamma}: F_{\mathrm{p}}[0,1] \rightarrow[0,1]$ in Prop IV.12 satisfies Cond. 2 in Def. III.10.

Proof. It is easy to see that Cond. $2 \mathrm{a}$ is satisfied.

We prove that Cond. $2 \mathrm{~b}$ is satisfied. Let $b_{1}, b_{2}: X \rightarrow[0,1]$ and assume that $b_{1} \leq b_{2}$. Let $x \in X$ and assume that $c(x)=$ $(\delta, t) \in F_{\mathrm{p}} X=\mathcal{D} X \times\{0,1\}$. Then we have:

$$
\begin{aligned}
& r_{\mathrm{nc}, \gamma} \circ F_{\mathrm{p}} b_{1}(\delta, t) \\
& = \begin{cases}1 & (t=1) \\
\sum_{x \in X} \delta(x) \cdot b_{1}(x) & (t=0)\end{cases} \\
& =\left\{\begin{array}{ll}
1 & (t=1) \\
\sum_{x \in X} \delta(x) \cdot b_{2}(x) & (t=0)
\end{array} \quad\left(\text { by } b_{1} \leq b_{2}\right)\right. \\
& =r_{\mathrm{nc}, \gamma} \circ F_{\mathrm{p}} b_{2}(\delta, t) \quad\left(\text { by def. of } r_{\mathrm{nc}, \gamma} \text { and } F_{\mathrm{p}}\right) \text {. }
\end{aligned}
$$

Therefore Cond. $2 \mathrm{~b}$ is satisfied.

Lemma A.24. The algebra $r_{\mathrm{nc}, \gamma}: F_{\mathrm{p}}[0,1] \rightarrow[0,1]$ in Prop. IV.12 is corecursive.

Proof. Let $c: X \rightarrow F_{\mathrm{p}} X$ be an $F_{\mathrm{p}}$-coalgebra. For each $x \in X$ such that $c(x)=(\delta, t) \in F_{\mathrm{p}} X$, we write $c_{1}(x)$ and $c_{2}(x)$ for $\delta \in \mathcal{D}[0,1]$ and $t \in\{0,1\}$ respectively. We prove that $\Phi_{c, r_{\mathrm{nc}, \gamma}}$ has a unique fixed point.

We first show that $\Phi_{c, r_{\mathrm{n} c}, \gamma}$ has a fixed point. By Lem. A.23. the poset $\left([0,1]^{X}, \leq\right)$ (here $\leq$ denotes the pointwise extension) is a complete lattice. Therefore we can construct a transfinite sequence

$$
\perp_{[0,1]} \leq \Phi_{c, r_{\mathrm{nc}, \gamma}}\left(\perp_{[0,1]}\right) \leq \cdots \leq \Phi_{c, r_{\mathrm{nc}, \gamma}}^{\mathfrak{a}}\left(\perp_{[0,1]}\right) \leq \cdots
$$

as in Thm. I.18 2. By Thm. I.18 2, there exists an ordinal $\mathfrak{n}$ such that $\Phi_{c, r_{\mathrm{nc}, \gamma}}^{\mathfrak{n}}\left(\perp_{[0,1]}\right)$ is the least fixed point of $\Phi_{c, r_{\mathrm{nc}, \gamma}}$. Let $f=\Phi_{c, r_{\mathrm{nc}, \gamma}}^{\mathfrak{n}}\left(\perp_{[0,1]}\right)$.

It remains to show that $f$ is the unique fixed point of $\Phi_{c, r_{\mathrm{nc}, \gamma}}$. Let $g: X \rightarrow[0,1]$ be a fixed point of $\Phi_{c, r_{\mathrm{nc}, \gamma}}$. As $f$ is the least fixed point, we have $f(x) \leq g(x)$ for each $x \in X$. We now define $h: X \rightarrow[0,1]$ by $h(x)=g(x)-f(x)$. Then we have:

$$
\begin{aligned}
& \sup _{x \in X} h(x) \\
& =\sup _{x \in X}(g(x)-f(x)) \quad \text { (by def. of } h \text { ) } \\
& =\sup _{x \in X}\left(\Phi_{c, r_{\mathrm{nc}, \gamma}}(g)(x)-\Phi_{c, r_{\mathrm{nc}, \gamma}}(f)(x)\right) \\
& \text { ( } f \text { and } g \text { are fixed points) } \\
& \leq \sup _{x \in X}\left(\gamma \cdot \sum_{x^{\prime} \in X} c_{1}(x)\left(x^{\prime}\right) \cdot g\left(x^{\prime}\right)\right. \\
& \left.-\gamma \cdot \sum_{x^{\prime} \in X} c_{1}(x)\left(x^{\prime}\right) \cdot f\left(x^{\prime}\right)\right) \\
& \left.c_{2}(x)=1 \Rightarrow \Phi_{c, r}(f)(x)=\Phi_{c, r}(g)(x)=1\right) \\
& =\gamma \cdot \sup _{x \in X} \sum_{x^{\prime} \in X} c_{1}(x)\left(x^{\prime}\right) \cdot\left(g\left(x^{\prime}\right)-f\left(x^{\prime}\right)\right) \\
& =\gamma \cdot \sup _{x \in X} \sum_{x^{\prime} \in X} c_{1}(x)\left(x^{\prime}\right) \cdot h\left(x^{\prime}\right) \quad \text { (by def. of } h \text { ) } \\
& \leq \gamma \cdot \sup _{x \in X} \sup _{x^{\prime} \in X} h\left(x^{\prime}\right) \quad\left(\text { by } \sum_{x^{\prime} \in X} c_{1}(x)\left(x^{\prime}\right) \leq 1\right) \\
& =\gamma \cdot \sup _{x \in X} h(x)
\end{aligned}
$$

As $0 \leq \gamma<1$, we have $\sup _{x \in X} h(x)=0$, and this implies $f=g$. This concludes the proof.

Proof (Prop. IV.12). We prove that $\left(r_{\mathrm{nc}, \gamma}, q_{\mathrm{nc}}, \leq\right)$ satisfies the conditions in Def. III.10.

Cond. 1]: Let $(\delta, t) \in F_{\mathrm{p}}[0,1]=\mathcal{D}[0,1] \times\{0,1\}$. Then we have:

$$
\begin{aligned}
& q_{\mathrm{nc}} \circ r_{\mathrm{nc}, \gamma}(\delta, t) \\
& = \begin{cases}q_{\mathrm{nc}}(1) & (t=1) \\
q_{\mathrm{nc}}\left(\gamma \cdot \sum_{a \in \operatorname{supp}(\delta)} a \cdot \delta(a)\right) & (t=0)\end{cases} \\
& = \begin{cases}1 & (t=1) \\
\gamma \cdot \sum_{a \in \operatorname{supp}(\delta)} a \cdot \delta(a) & (t=0)\end{cases} \\
& \leq \begin{cases}1 & \text { (by def. of } \left.r_{\mathrm{nc}, \gamma}\right) \\
\sum_{a \in \operatorname{supp}(\delta)} a \cdot \delta(a) & (t=0)\end{cases} \\
& =\sigma_{\mathrm{p}}(\delta, t) \\
& =\sigma_{\mathrm{p}} \circ F_{\mathrm{p}} q_{\mathrm{nc}}(\delta, t)
\end{aligned}
$$

Hence Cond. 1 is satisfied. 
Cond. 2. We have already proved in Lem. A.23.

Cond. 3. Immediate from $q_{\mathrm{nc}}=\mathrm{id}_{[0,1]}$.

Cond. 4. It is already proved in Lem. A.24.

Hence $\left(r_{\mathrm{nc}, \gamma}, q_{\mathrm{p}}, \leq\right)$ is a ranking domain.

\section{Towards Further Examples}

Now that we have a general categorical axiomatization of ranking functions ( $(\mathrm{II})$, we would like to exploit it in deriving further examples of "ranking functions" that are previously unknown, hoping that they will provide novel proof methods for various liveness properties. In the previous section we derived two variations of ranking supermartingales. Towards further examples, here we indicate a possible direction.

We can say that not many concrete examples are known of corecursive algebras. Nevertheless, the following "closure properties" can be used to derive new examples.

Lemma A.25. Let $F: \mathbb{C} \rightarrow \mathbb{C}$ be a functor.

1) ([33]) Consider the well-known construction of the final sequence: $1 \stackrel{!}{\leftarrow} F 1 \stackrel{F !}{\leftarrow} F^{2} 1 \stackrel{F^{2} !}{\leftarrow} \cdots \leftarrow F^{\mathfrak{a}} 1 \leftarrow \cdots$, where we define $F^{\mathfrak{a}} 1$ for an arbitrary ordinal a (we assume enough limits and let $F^{\mathfrak{a}} 1=\lim _{\mathfrak{b}<\mathfrak{a}} F^{\mathfrak{b}} 1$ for a limit ordinal $\mathfrak{a})$. Then, for each $\mathfrak{a}$, the algebra $F^{\mathfrak{a}}$ !: $F\left(F^{\mathfrak{a}} 1\right) \rightarrow F^{\mathfrak{a}} 1$ is corecursive.

2) (dual of [34]) Let $\tau: F \Rightarrow G$ be a natural transformation. Then a corecursive $G$-algebra $s: G R \rightarrow R$ induces a corecursive F-algebra $s \circ \tau_{R}: F R \rightarrow R$.

Lem. A.25112 together suggest the following workflow. For dynamical systems modeled as $F$-coalgebras, we pick a natural transformation $\tau: F \Rightarrow G$ and an ordinal $\mathfrak{a}-$ the former abstracts (or collapses) $F$-behaviors into $G$-behaviors that are supposedly simpler. We then use the set $G^{\mathfrak{a}} 1$ of " $G$ behaviors up-to $\mathfrak{a}$ " as the ranking domain. The set carries a corecursive $G$-algebra by Lem. A.25]1, and via $\tau$ it carries a corecursive $F$-algebra, too (Lem. A.25|2).

An example of such "behavioral" ranking domains is in The set Tree fin $\stackrel{\perp}{ }$ consists of: (unlabeled) trees that are possibly countably branching and of finite depth; and the special element $\perp$ that designates non-well-foundedness. It is shown that, for the problem of universal reachability of tree automata, we can indeed use the set Tree fin to form a (categorical) ranking domain. Here we use the functors $F=\left(\coprod_{n \in \mathbb{N} \cup\{\omega\}} \Sigma_{n} \times\right.$ $\left.\left(\_\right)^{n}\right) \times\{0,1\}$ and $G=1+\bigsqcup_{n \in \mathbb{N} \cup\{\omega\}}\left({ }_{-}\right)^{n}$, and $\tau: F \Rightarrow G$ collapses the elements $(t, 1) \in F X$-i.e. accepting states-to the unique element of 1 in $G X$.

In fact the set Tree ${ }_{\text {fin }}^{\perp}$ is not precisely the outcome $G^{\omega} 1$ of the workflow described above: $G^{\omega} 1$ contains all the finite and infinite trees (with suitable branching degrees) as separate elements; but in Tree $\stackrel{\perp}{\perp}$ a single element $\perp$ stands for all the "non-well-founded" trees that contains at least one infinite branch. A categorical description of such collapse is our future work; so is a general order with which we can equip $G^{\omega} 1$.

\section{A Ranking Domain for Tree Automata}

Notation A.26. We write \langle\rangle for the empty sequence in $\mathbb{N}^{*}$. For $w, w^{\prime} \in \mathbb{N}^{*} \cup \mathbb{N}^{\omega}$, we write $w \preceq w^{\prime}$ if $w$ is a prefix of $w^{\prime}$.

Definition A.27 (unlabeled tree). An unlabeled tree is a set $D \subseteq \mathbb{N}^{*}$ that satisfies the following conditions.

1) The empty sequence is in $D$, i.e. \langle\rangle$\in D$.

2) The set $D$ is prefix-closed, i.e. $\forall w \in \mathbb{N}^{*} . \forall i \in \mathbb{N}$. $w i \in$ $D \Rightarrow w \in D$.

3) The set $D$ is downward-closed, i.e $\forall w \in \mathbb{N}^{*}$. $\forall i \in$ $\mathbb{N} . \forall j \leq i . w i \in D \Rightarrow w j \in T$.

An unlabeled tree $D$ is said to be finite-depth if it satisfies the following additional condition.

4) The set $D$ has no strictly increasing sequence with respect to $\preceq$, i.e.

$$
\forall v \in \mathbb{N}^{\omega} .|\{w \in D \mid w \preceq v\}|<\infty .
$$

We write Tree (resp. Tree ${ }_{\text {fin }}$ ) for the set of all unlabeled trees (resp. unlabeled finite-depth trees).

For an unlabeled tree $D$, we define a function branch $_{D}$ : $D \rightarrow \mathbb{N} \cup\{\infty\}$ as follows.

$$
\operatorname{branch}_{D}(w)=\max \{i \mid w i \in D\} .
$$

The prefix order over Tree is a partial order $\preceq$ that is defined by

$D_{1} \preceq D_{2} \stackrel{\text { def. }}{\Leftrightarrow}\left(\begin{array}{l}D_{1} \subseteq D_{2}, \text { and } \\ \forall w \in D_{1} . \text { branch }_{D_{1}}(w) \in\left\{0, \operatorname{branch}_{D_{2}}(w)\right\}\end{array}\right)$.

For a (possibly infinite) family of unlabeled trees $\left(D_{i} \in\right.$ Tree $)_{0 \leq i<\mathfrak{a}}$ where $\mathfrak{a} \leq \omega$, we define a new tree combine $\left(D_{1}, D_{2}, \ldots\right) \subseteq \mathbb{N}^{*}$ as follows.

$$
\text { combine }\left(D_{1}, D_{2}, \ldots\right)=\{\langle\rangle\} \cup\left\{i w \mid i<\mathfrak{a}, w \in D_{i}\right\}
$$

Remark A.28. We note that $D \in$ Tree $_{\text {fin }}$ is assumed to be finite-depth, but it is not assumed to be finitely-branching. For example, we have $(\{\langle\rangle\} \cup\{\langle n\rangle \mid n \in \mathbb{N}\}) \in$ Tree $_{\text {fin }}$ where $\langle n\rangle$ denotes a sequence whose length is 1 .

Definition A.29 (ranked alphabet). A ranked alphabet is a pair $\Sigma=\left(\Sigma,\left.\right|_{-} \mid\right)$of a set $\Sigma$ and an arity function $\left.\right|_{-} \mid$: $\Sigma \rightarrow \mathbb{N} \cup\{\infty\}$. For each $a \in \Sigma,|a| \in \mathbb{N} \cup\{\infty\}$ is called the arity of $a$. For each $n \in \mathbb{N} \cup\{\infty\}$, we write $\Sigma_{n}$ for $\{a \in \Sigma|| a \mid=n\} \subseteq \Sigma$.

Definition A.30 (labeled tree). Let $\Sigma$ be a ranked alphabet. A $\Sigma$-labeled tree is a pair $T=(D, l)$ of an unlabeled tree $D \subseteq$ Tree and a labeling function $l: D \rightarrow \Sigma$ that respects the arity, i.e.

$$
\forall w \in D . l(w)=\operatorname{branch}_{D}(w) .
$$

The unlabeled tree $D$ is called the domain of $T$. We write Tree $_{\Sigma}$ for the set of all $\Sigma$-labeled trees.

Definition A.31 $\left(F_{\mathrm{t}, \Sigma}\right)$. Let $\Sigma$ be a ranked alphabet. We define a functor $F_{\mathrm{t}, \Sigma}:$ Sets $\rightarrow$ Sets by

$$
F_{\mathrm{t}, \Sigma}=\left(\coprod_{n \in \mathbb{N} \cup\{\omega\}} \Sigma_{n} \times\left({ }_{-}\right)^{n}\right) \times\{0,1\} .
$$


Notation A.32. Let $c: X \rightarrow F_{\mathrm{t}, \Sigma} X$ be an $F_{\mathrm{t}, \Sigma}$-coalgebra. For each $x \in X$ where $c(x)=(\xi, t)$, we write $c_{1}(x)$ and $c_{2}(x)$ for $\xi \in \coprod_{n \in \mathbb{N} \cup\{\omega\}} \Sigma_{n} \times\left({ }_{-}\right)^{n}$ and $t \in\{0,1\}$, respectively.

Definition $\mathbf{A . 3 3}$ (run tree). Let $c: X \rightarrow F_{\mathrm{t}, \Sigma} X$ be an $F_{\mathrm{t}, \Sigma^{-}}$ coalgebra. We define a new ranked alphabet $X \times \Sigma=(X \times$ $\left.\Sigma,\left.\right|_{-} \mid\right)$by $|(x, a)|=|a|$. For $x \in X$, an $(X \times \Sigma)$-labeled tree $(D, l)$ is called a run tree of $c$ from $x$ if it satisfies the following conditions (here for each $w \in D$ where $l(w)=\left(x^{\prime}, a\right), l_{1}(w)$ and $l_{2}(w)$ denote $x^{\prime}$ and $a$ respectively):

1) $l_{1}(\langle\rangle)=x$; and

2) for each $w \in D$,

$$
c_{1}\left(l_{1}(w)\right)=\left(l_{2}(w), l_{1}(w 0), l_{1}(w 1), \ldots\right) .
$$

A run tree $(D, l)$ is called accepting if it has no infinite branch labeled only with non-accepting states, i.e.

$\neg\left(\exists v \in \mathbb{N}^{\omega} . \forall w \in \mathbb{N}^{*}\right.$ s.t. $\left.w \preceq v .\left(w \in D \wedge c_{2}(l(w))=0\right)\right)$.

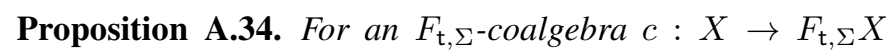
and $x \in X$, there exists a unique run tree of $c$ from $x$.

Definition A.35 (RunTree ${ }_{c}$ and Reach $_{c}$ ). By Prop. A.34, we write $\operatorname{RunTree}_{c}(x)$ for the unique run tree of $c$ from $x$. For an $F_{\mathrm{t}, \Sigma}$-coalgebra $c: X \rightarrow F_{\mathrm{t}, \Sigma} X$, we define a function $\operatorname{Reach}_{c}$ : $X \rightarrow\{0,1\}$ by

$$
\operatorname{Reach}_{c}(x)= \begin{cases}1 & \left(\operatorname{RunTree}_{c}(x) \text { is accepting }\right) \\ 0 & (\text { otherwise })\end{cases}
$$

Definition A.36. We define an $F_{\mathrm{t}, \Sigma}$-modality $\sigma_{\mathrm{t}}$ : $F_{\mathrm{t}, \Sigma}\{0,1\} \rightarrow\{0,1\}$ over a truth-value domain $(\{0,1\}, \leq)$ by

$$
\sigma_{\mathrm{t}}\left(\left(a, p_{1}, p_{2}, \ldots\right), t\right)= \begin{cases}1 & \left(t=1 \text { or } \forall i \cdot p_{i}=1\right) \\ 0 & (\text { otherwise })\end{cases}
$$

Proposition A.37. The $F_{\mathrm{t}, \Sigma}$-modality $\sigma_{\mathrm{t}}: F_{\mathrm{t}, \Sigma}\{0,1\} \rightarrow$ $\{0,1\}$ in Def. A.36 has least fixed points. For an $F_{\mathrm{t}, \Sigma^{-}}$ coalgebra $c: X \rightarrow F_{\mathrm{t}, \Sigma} X$, the (coalgebraic) least fixed property $\llbracket \mu \sigma_{\mathrm{t}} \rrbracket_{c}: X \rightarrow\{0,1\}$ is given by the function Reach $_{c}: X \rightarrow\{0,1\}$ in Def. A.35.

Proof. We define $f: X \rightarrow\{0,1\}$ by $f=$ Reach $_{c}$. It suffices to prove that $f$ is the least fixed-point of $\Phi_{c, \sigma_{\mathrm{t}}}:\left(\operatorname{Tree}_{\mathrm{fin}}^{\perp}\right)^{X} \rightarrow$ $\left(\text { Tree }_{\text {fin }}^{\perp}\right)^{X}$.

We first show that $f$ is a fixed point of $\Phi_{c, \mathrm{t}}$. Let $x \in X$ and assume that $c(x)=\left(\left(a, x_{1}, x_{2}, \ldots\right), t\right) \in F_{\mathrm{t}, \Sigma} X$. Then we have:

$$
\begin{aligned}
& \sigma_{\mathrm{t}} \circ F_{\mathrm{t}, \Sigma} f\left(\left(a, x_{1}, x_{2}, \ldots\right), t\right) \\
& = \begin{cases}1 & \left.\left(t=1 \text { or } \forall i . f\left(x_{i}\right)=1\right) \quad \text { (by def. of } \sigma_{\mathrm{t}}\right) \\
0 & \text { (otherwise) }\end{cases} \\
& = \begin{cases}1 & \left(t=1 \text { or } \forall i . \text { RunTree }_{c}\left(x_{i}\right)\right. \text { is accepting) } \\
0 & \text { (otherwise) }\end{cases}
\end{aligned}
$$

(by def. of $f$ )

$$
= \begin{cases}1 & \left(\operatorname{RunTree}_{c}(x) \text { is accepting }\right) \\ 0 & \text { (otherwise) }\end{cases}
$$

$$
=f(x) \text {. }
$$

(by def. of RunTree )

Hence $f$ is a fixed point of $\Phi_{c, \sigma_{\mathrm{t}}}$.

It remains to show that $f$ is the least fixed point. Let $f^{\prime}$ : $X \rightarrow\{0,1\}$ be a fixed point of $\Phi_{c, \sigma_{t}}$. It suffices to prove that for each $x \in X, f^{\prime}(x)=0$ implies $f(x)=0$.

Let $x \in X$ and assume $f^{\prime}(x)=0$. Let $(D, l)=$ $\operatorname{RunTree}_{c}(x)$. For each $i \in \mathbb{N}$, we inductively define $x_{i} \in X$ and $v_{i} \in \mathbb{N}$ so that $f^{\prime}\left(x_{i}\right)=0$ for each $i$ as follows.

- For $i=0$, we let $x_{0}=x$. Then we have $f^{\prime}\left(x_{0}\right)=f(x)=$ 0.

- Let $i \geq 0$ and assume that we have defined $x_{j}$ and $v_{j-1}$ for each $j \leq i$ so that $f^{\prime}\left(x_{i}\right)=0$. Let $c\left(x_{i}\right)=$ $\left(\left(a_{i}, x_{i, 1}, x_{i, 2}, \ldots\right), t_{i}\right)$. As $f^{\prime}$ is a fixed point of $\Phi_{c, \sigma_{\mathrm{t}}}$, we have:

$$
0=f^{\prime}\left(x_{i}\right)=\left(\sigma_{\mathrm{t}} \circ F_{\mathrm{t}, \Sigma} f^{\prime} \circ c\right)\left(x_{i}\right) .
$$

By the definition $\sigma_{\mathrm{t}}$, this means that there exists $k$ such that $f^{\prime}\left(x_{i, k}\right)=0$. We let $v_{i}=k$ and $x_{i+1}=x_{i, k}$.

We can prove that $x_{i}$ and $v_{i}$ satisfy the followings.

1) $\forall i .\left(v_{0} \ldots v_{i-1}\right) \in D$

2) $\forall i . l\left(v_{0} \ldots v_{i-1}\right)=x_{i}$

3) $\forall i . c_{2}\left(l\left(v_{0} \ldots v_{i-1}\right)\right)=0$

Here (1) and (2) are immediate from the definition. The equation (15) implies that $c_{2}\left(l\left(v_{0} \ldots v_{i-1}\right)\right)=c_{2}\left(x_{i}\right)=t_{i}=0$ for each $i \in \mathbb{N}$.

This means that $\operatorname{RunTree}_{c}(x)$ is not accepting (see Def. A.33, and therefore we have $f(x)=0$. This concludes the proof.

Proposition A.38. The $F_{\mathrm{t}}$-modality $\sigma_{\mathrm{t}}: F_{\mathrm{t}}\{0,1\} \rightarrow\{0,1\}$ in Def. A.36 satisfies conditions in Asm. III.7

Proposition A.39. Let $\Sigma$ be a ranked alphabet and $\operatorname{Tree}_{\text {fin }}^{\perp}=$ Tree $_{\text {fin }} \cup\left\{\perp_{\mathrm{t}}\right\}$. We define an $F_{\mathrm{t}, \Sigma}$-algebra $r_{\mathrm{t}}: F_{\mathrm{t}, \Sigma}\left(\right.$ Tree $\left._{\text {fin }}^{\perp}\right) \rightarrow$ Tree ${ }_{\text {fin }}^{\perp}$, a function $q_{\mathrm{t}}:$ Tree $_{\text {fin }}^{\perp} \rightarrow\{0,1\}$ and a partial order $\sqsubseteq_{\text {Tree }_{\text {fin }}^{\perp}}^{\perp}$ over Tree $\operatorname{fin}_{\text {fin }}^{\perp}$ as follows.

1) $r_{\mathrm{t}}\left(\left(a, D_{1}, D_{2}, \ldots\right), t\right)$

$$
= \begin{cases}\langle\rangle & (t=1) \\ \text { combine }\left(D_{1}, D_{2}, \ldots\right) & \left(t=0 \text { and } \forall i . D_{i} \neq \perp_{\mathrm{t}}\right) \\ \perp_{\mathrm{t}} & \left(t=0 \text { and } \exists i . D_{i}=\perp_{\mathrm{t}}\right)\end{cases}
$$


2) $q_{\mathrm{b}}(D)= \begin{cases}1 & \left(D \neq \perp_{\mathrm{t}}\right) \\ 0 & \left(D=\perp_{\mathrm{t}}\right)\end{cases}$

3) $D_{1} \sqsubseteq$ Tree $_{\frac{1}{\text { fin }}}^{\perp} D_{2} \stackrel{\text { def. }}{\Leftrightarrow} D_{1}=\perp_{\mathrm{t}}$ or $\left(D_{1}, D_{2} \in\right.$

Tree $_{\text {fin }}$ and $D_{1} \succeq D_{2}$ ) (note the direction).

Then $\left(r_{\mathrm{t}}, q_{\mathrm{t}}, \sqsubseteq_{\text {Tree }_{\mathrm{fin}}^{\perp}}^{\perp}\right)$ is a ranking domain.

Lemma A.40. The poset $\left(\operatorname{Tree}_{\mathrm{fin}}^{\perp}, \sqsubseteq_{\mathrm{Tree}_{\mathrm{fin}}}^{\perp}\right)$ is a complete lattice.

Proof. Let $K \subseteq$ Tree $_{\text {fin }}^{\perp}$.

$K$ has the least upper bound. We first prove that $K$ has the least upper bound. If $K \cap \operatorname{Tree}_{\text {fin }}=\emptyset$, then $\perp_{t}$ is the least upper bound of $K$.

Assume $K \cap$ Tree $_{\text {fin }} \neq \emptyset$. If $K^{\prime}=K \backslash\left\{\perp_{\mathrm{t}}\right\}$ has the least upper bound, then it is also the least upper bound of $K$.

We define $A \subseteq \mathbb{N}^{*}$ as follows.

$$
A=\bigcup\left\{D^{\prime} \in \text { Tree }_{\text {fin }} \mid \forall D \in K^{\prime} . D^{\prime} \preceq D\right\} .
$$

As $\{\langle\rangle\} \preceq D$ holds for each $D \in K^{\prime}$ and Cond. 1 13 in Def. A.27 are preserved by the union, $A \in$ Tree. We prove that $A$ is the least upper bound of $K^{\prime}$.

We first prove that $A$ is an upper bound. Let $D \in K^{\prime}$. We prove $D \sqsubseteq$ Tree $_{\text {fin }}^{\perp} A$. To this end, by the definition of $\preceq$, it suffices to prove $A \subseteq D$ and $\operatorname{branch}_{A}(w) \in\left\{0, \operatorname{branch}_{D}(w)\right\}$ for each $w \in A$. The former is immediate from the definition of $A$. The latter is satisfied because we have $\operatorname{branch}_{D^{\prime}}(w) \in$ $\left\{0, \operatorname{branch}_{D}(w)\right\}$ for each $D^{\prime}$ such that $D^{\prime} \preceq D$ and $w \in D^{\prime}$.

Hence $A$ is an upper bound of $K$. We note that this also proves that $A$ is finite-depth.

It is immediate from the definition of $A$ that $A$ is the least upper bound.

$K$ has the greatest lower bound. We prove that $K$ has the greatest lower bound. If $\perp_{\mathrm{t}} \in K$, then $\prod K=\perp_{\mathrm{t}}$. If $K=\emptyset$, then $\Pi K=\{\langle\rangle\}$.

We assume $\perp_{\mathrm{t}} \notin K$ and $K \neq \emptyset$. For $D_{1}, D_{2} \in K$, if there exists $D_{3} \in K$ such that $D_{1} \preceq D_{3}$ and $D_{2} \preceq D_{3}$ then by the definition of $\preceq$, we have:

$$
\begin{array}{r}
\left.\forall w \in D_{1} \cap D_{2} . \operatorname{branch}_{D_{1}}(w) \neq 0 \text { and } \operatorname{branch}_{D_{2}}(w) \neq 0\right) \\
\Rightarrow \operatorname{branch}_{D_{1}}(w)=\operatorname{branch}_{D_{2}}(w) .
\end{array}
$$

Therefore if there exists $D_{1}, D_{2} \in K$ that satisfy

$$
\begin{array}{r}
\exists w \in D_{1} \cap D_{2} . \operatorname{branch}_{D_{1}}(w) \neq 0, \operatorname{branch}_{D_{2}}(w) \neq 0 \\
\text { and } \operatorname{branch}_{D_{1}}(w) \neq \operatorname{branch}_{D_{2}}(w),
\end{array}
$$

then there does not exist $D_{3} \in K$ such that $D_{1} \preceq D_{3}$ and $D_{2} \preceq D_{3}$. This implies $\sqcap K=\perp_{\mathrm{t}}$.

Assume that there does not exist $D_{1}, D_{2} \in K$ that satisfy (16). We define $B \in \mathbb{N}^{*}$ by

$$
B=\bigcup K \text {. }
$$

It is easy to see that $B \in$ Tree. It is also easy to see that if $B$ is not finite-depth then $\perp_{t}$ is the greatest lower bound of $K$.

Assume $B \in$ Tree $_{\text {fin }}$. We prove that $B$ is the greatest lower bound of $K$. Let $D \in K$. By the definition of $B$, we have $D \subseteq B$. As there exist no $D_{1}, D_{2} \in K$ that satisfy (16), we have $\operatorname{branch}_{D}(w) \in\left\{0, \operatorname{branch}_{B}(w)\right\}$ for each $w \in D$. Hence we have $B \sqsubseteq_{\text {Tree }_{\mathrm{fin}}^{\perp}}^{\perp} D$, and therefore $B$ is a lower bound of $K$. It is immediate from its definition that $B$ is the greatest lower bound.

Lemma A.41. The triple $\left(r_{\mathrm{t}}, q_{\mathrm{t}}, \sqsubseteq_{\text {Tree }_{\mathrm{fin}}^{+}}^{+}\right)$in Prop. A.39 satisfies Cond. 2 in Def. III.10

Proof. Cond. 2a is immediate from Lem. A.40.

It is easy to see that Cond. $2 \mathrm{~b}$ is satisfied.

Lemma A.42. The algebra $r_{\mathrm{t}}: F_{\mathrm{t}, \Sigma} \operatorname{Tree}_{\mathrm{fin}}^{\perp} \rightarrow \operatorname{Tree}_{\mathrm{fin}}^{\perp}$ in Prop. A.39 is a corecursive algebra.

Proof. It suffices to show that the function $\Phi_{c, r_{\mathrm{t}}}$ : $\left(\text { Tree }_{\text {fin }}^{\perp}\right)^{X} \rightarrow\left(\text { Tree }_{\text {fin }}^{\perp}\right)^{X}$ has a unique fixed point.

By Lem. A.41, the set $\left(\left(\text { Tree }_{f i n}^{\perp}\right)^{X}\right.$, $\left.\Xi_{\text {Tree }_{\text {fin }}^{\perp}}^{\perp}\right)$ where $\sqsubseteq_{\text {Tree }_{\text {fin }}^{\perp}}$ denotes the pointwise extension is a complete lattice. Therefore by Thm. II.18, the function $\Phi_{c, r_{\mathrm{t}}}$, which is monotone by Lem. A.41, has the least fixed point.

It remains to show that this is the unique fixed point. Let $f, f^{\prime}: X \rightarrow$ Tree $_{\text {fin }}^{\perp}$ be fixed points of $\Phi_{c, r_{\mathrm{t}}}$.

Let $g: X \rightarrow$ Tree $_{\text {fin }}^{\perp}$ and assume $g(x)=\perp_{\mathrm{t}}$. Let $c(x)=$ $\left(\left(a, x_{0}, x_{1}, \ldots\right), t\right)$. As $g$ is a fixed point of $\Phi_{c, r_{\mathrm{t}}}$, we have $\left(r_{\mathrm{t}} \circ F_{\mathrm{t}, \Sigma} g \circ c\right)(x)=\perp_{\mathrm{t}}$. Hence we have $t=0$ and that there exists $i$ such that $g\left(x_{i}\right)=\perp_{\mathrm{t}}$.

Therefore we can inductively define a sequence $x^{0} x^{1} x^{2} \ldots \in$ $X^{\omega}$ so that:

1) $g\left(x^{i}\right)=\perp_{t}$;

2) $c_{2}\left(x^{i}\right)=0$ for each $i \in \omega$; and

3) $c_{1}\left(x^{i}\right)=\left(a^{i}, x_{1}^{i}, x_{2}^{i}, \ldots\right)$ then $x^{i+1}=x_{n}^{i}$ for some $n$.

Conversely, we can prove that existence of a sequence $x^{0} x^{1} x^{2} \ldots \in X^{\omega}$ that satisfies the conditions above implies $g(x)=\perp_{\mathrm{t}}$.

Therefore we have: $f(x)=\perp_{\mathrm{t}}$ if and only if $f^{\prime}(x)=\perp_{\mathrm{t}}$.

We now prove that for each $n \in \mathbb{N}, w \in \mathbb{N}^{n}$ and $x \in X$ such that $f(x) \neq \perp_{\mathrm{t}}$ and $f^{\prime}(x) \neq \perp_{\mathrm{t}}, w \in f(x)$ if and only if $w \in f^{\prime}(x)$ by the induction on $n$.

For $n=0$, by Def. A.27 we have \langle\rangle$\in f(x)$ and \langle\rangle$\in f^{\prime}(x)$ for each $x$.

Let $n>0$ and assume that for each $w \in \mathbb{N}^{n}$ and $x \in X$ such that $f(x) \neq \perp_{\mathrm{t}}$ and $f^{\prime}(x) \neq \perp_{\mathrm{t}}$, we have $w \in f(x)$ if and only if $w \in f^{\prime}(x)$. Let $x \in X$ and assume $c(x)=$ $\left(\left(a, x_{0}, x_{1}, \ldots\right), t\right) \in\left(\Sigma \times\left({ }_{-}\right)^{m}\right) \times\{0,1\}$. For each $i \in \mathbb{N}$, we have:

$$
\begin{array}{lr}
i w \in f(x) & \\
\Leftrightarrow i w \in \Phi_{c, r_{\mathrm{t}}}(f) & (f \text { is a fixed point) } \\
\Leftrightarrow i<m \text { and } w \in f\left(x_{i}\right) & \text { (by def. of } \left.r_{\mathrm{t}}\right) \\
\Leftrightarrow i<m \text { and } w \in f^{\prime}\left(x_{i}\right) & \text { (by IH) } \\
\Leftrightarrow i w \in \Phi_{c, r_{\mathrm{t}}}\left(f^{\prime}\right) & \text { (by def. of } \left.r_{\mathrm{t}}\right) \\
\Leftrightarrow i w \in f^{\prime}(x) & \left(f^{\prime}\right. \text { is a fixed point). }
\end{array}
$$


Hence we have $f(x)=f^{\prime}(x)$ for each $x \in X$. This concludes the proof.

Proof (Prop. A.39). We prove that $\left(r_{\mathrm{t}}, q_{\mathrm{t}}, \sqsubseteq_{\mathrm{Tree}_{\mathrm{fin}}}^{\frac{1}{\mathrm{fin}}}\right)$ satisfies conditions in Def. III.10.

17. Let $\left(\left(a, D_{0}, D_{1}, \ldots\right), t\right) \in F_{\mathrm{t}, \Sigma} \operatorname{Tree}_{\mathrm{fin}}^{\perp}$. Then we have:

$\left(q_{\mathrm{t}} \circ r_{\mathrm{t}}\right)\left(\left(a, D_{0}, D_{1}, \ldots\right), t\right)=1$

$\Leftrightarrow r_{\mathrm{t}}\left(\left(a, D_{0}, D_{1}, \ldots\right), t\right) \neq \perp_{\mathrm{t}} \quad$ (by def. of $q_{\mathrm{t}}$ )

$\Leftrightarrow t=1$ or $\forall i . D_{i} \neq \perp_{\mathrm{t}} \quad$ (by def. of $r_{\mathrm{t}}$ )

$\Leftrightarrow t=1$ or $\forall i . q_{\mathrm{t}}\left(D_{i}\right)=1 \quad$ (by def. of $q_{\mathrm{t}}$ )

$\Leftrightarrow\left(\sigma_{\mathrm{t}} \circ F_{\mathrm{t}, \Sigma} q_{\mathrm{t}}\right)\left(\left(a, D_{0}, D_{1}, \ldots\right), t\right)=1 \quad$ (by def. of $\left.\sigma_{\mathrm{t}}\right)$.

Hence we have $q_{\mathrm{t}} \circ r_{\mathrm{t}}=\sigma \circ F_{\mathrm{t}, \Sigma} q_{\mathrm{t}}$.

2. We have already proved in Lem. A.41

3. Monotonicity and strictness are immediate from the definition of $q_{\mathrm{t}}$. We prove that $q_{\mathrm{t}}$ is continuous. Let $K \subseteq \operatorname{Tree}_{\text {fin }}^{\perp}$. Then we have:

$$
\begin{array}{ll}
\bigsqcup_{D \in K} q_{\mathrm{t}}(D)=0 & \\
\Leftrightarrow \forall D \in K \cdot q_{\mathrm{t}}(D)=0 & \\
\Leftrightarrow K=\left\{\perp_{\mathrm{t}}\right\} & \text { (by def. of } q_{\mathrm{t}} \text { ) } \\
\Leftrightarrow \bigsqcup K=\perp_{\mathrm{t}} & \text { (by def. of } \left.q_{\mathrm{t}}\right) .
\end{array}
$$

Hence $q_{\mathrm{t}}$ is continuous.

44. We have already proved in Lem. A.42

Proposition $\mathbf{A . 4 3}$ (completeness). For an arbitrary $F_{\mathrm{t}, \Sigma^{-}}$ coalgebra $c: X \rightarrow F_{\mathrm{t}, \Sigma} X$, there exists a ranking arrow $b: X \rightarrow$ Tree $_{\text {fin }}^{\perp}$ with respect to the ranking domain $\left(r_{\mathrm{t}}, q_{\mathrm{t}}, \sqsubseteq_{\text {Tree }_{\mathrm{fin}}^{\perp}}\right)$ in Prop. A.39 that satisfies the following.

$$
q_{\mathrm{t}} \circ b=\llbracket \mu \sigma_{\mathrm{t}} \rrbracket_{c}
$$

Proof. Immediate from $q_{\mathrm{t}} \circ r_{\mathrm{t}}=\sigma_{\mathrm{t}} \circ F_{\mathrm{t}, \Sigma} q_{\mathrm{t}}$ (see the proof of Prop. A.39) and Prop. III.18 